\title{
Gait characteristics of patients with COPD
}

Citation for published version (APA):

Liu, W. (2019). Gait characteristics of patients with COPD. [Doctoral Thesis, Maastricht University]. ProefschriftMaken Maastricht. https://doi.org/10.26481/dis.20191121wl

Document status and date:

Published: 21/11/2019

DOI:

10.26481/dis.20191121wl

Document Version:

Publisher's PDF, also known as Version of record

\section{Please check the document version of this publication:}

- A submitted manuscript is the version of the article upon submission and before peer-review. There can be important differences between the submitted version and the official published version of record.

People interested in the research are advised to contact the author for the final version of the publication, or visit the DOI to the publisher's website.

- The final author version and the galley proof are versions of the publication after peer review.

- The final published version features the final layout of the paper including the volume, issue and page numbers.

Link to publication

\footnotetext{
General rights rights.

- You may freely distribute the URL identifying the publication in the public portal. please follow below link for the End User Agreement:

www.umlib.nl/taverne-license

Take down policy

If you believe that this document breaches copyright please contact us at:

repository@maastrichtuniversity.nl

providing details and we will investigate your claim.
}

Copyright and moral rights for the publications made accessible in the public portal are retained by the authors and/or other copyright owners and it is a condition of accessing publications that users recognise and abide by the legal requirements associated with these

- Users may download and print one copy of any publication from the public portal for the purpose of private study or research.

- You may not further distribute the material or use it for any profit-making activity or commercial gain

If the publication is distributed under the terms of Article $25 \mathrm{fa}$ of the Dutch Copyright Act, indicated by the "Taverne" license above, 
Gait characteristics of patients with COPD 
(c) W.Y. Liu, Maastricht 2019. All rights reserved.

ISBN 978-94-6380-505-6

Typeset using $\mathrm{ET}_{\mathrm{E}} \mathrm{X}$

Printing ProefschriftMaken | www.proefschriftmaken.nl

Cover design M.J.M.M. Hoeijmakers

Layout design W.Y. Liu

The research presented in this thesis was performed at CIRO, Horn, the Netherlands; the Department of Nutrition and Movement Sciences, NUTRIM School of Nutrition and Translational Research in Metabolism, Maastricht University Medical Centre+, the Netherlands; and the Department of Biomechanics, Center of Human Movement Variability, University of Nebraska at Omaha, USA.

This thesis was financially supported by: CIRO, Horn, the Netherlands; and Maastricht University, Maastricht, the Netherlands. 


\title{
Gait characteristics of patients with COPD
}

\author{
PROEFSCHRIFT
}

Ter verkrijging van de graad van doctor aan de Universiteit Maastricht,

op gezag van de Rector Magnificus, Prof. dr. Rianne M. Letschert, volgens het besluit van het College van Decanen,

in het openbaar te verdedigen op donderdag 21 november 2019 om 16.00 uur

door

Wai Yan Liu 


\section{Promotoren}

Prof. dr. M.A. Spruit

Prof. dr. E.F.M. Wouters

\section{Copromotor}

Dr. K. Meijer

\section{Beoordelingscommissie}

Prof. dr. H.H.C.M. Savelberg (voorzitter)

Dr. A.J. van 't Hul, Radboud Universitair Medisch Centrum

Prof. dr. A.F. Lenssen

Prof. dr. M.A.G.M. Pijnappels, Vrije Universiteit Amsterdam

Prof. dr. L.W. van Rhijn 


\section{Contents}

Chapter 1. General introduction 1

Chapter 2. Reproducibility and validity of the 6-minute walk test using the Gait Real-time Analysis Interactive Lab in patients with COPD and healthy elderly

Chapter 3. Spatiotemporal gait characteristics in patients with COPD during the Gait Real-time Analysis Interactive Lab-based 6-minute walk test

Chapter 4. Alterations in stride-to-stride fluctuations in patients with chronic obstructive pulmonary disease during the self-paced treadmill 6-minute walk test

Chapter 5. Patients with COPD walk with less consistent organization of movement patterns of the lower extremity

Chapter 6. Effects of pulmonary rehabilitation on gait characteristics in patients with COPD

Chapter 7. General discussion

Summary

Samenvatting

143

Valorisation

Dankwoord

Curriculum vitae

155

List of publications 

CHAPTER

General introduction 


\section{Chronic obstructive pulmonary disease}

Chronic obstructive pulmonary disease (COPD) is defined as "a common preventable and treatable disease, characterized by persistent respiratory symptoms and airflow limitation that is due to airway and/or alveolar abnormalities usually caused by significant exposure to noxious particles or gases" [1]. A combination of small airway disease (obstructive bronchiolitis) and parenchymal destruction (emphysema) results in chronic airflow limitation. Chronic inflammation causes structural changes, which impair the ability of the airways to remain open during expiration. The loss of small airways may in turn result in airflow limitation and mucociliary dysfunction [1]. Moreover, there is heterogeneity between patients with COPD in terms of respiratory symptoms, frequency of exacerbations, lung function decline, response to therapy and survival [2].

COPD is a major cause of morbidity and mortality throughout the world [3]. According to the World Health Organization, COPD will become the third leading cause of death worldwide by 2030 [4]. The COPD burden is expected to increase due to continued exposure to COPD risk factors, such as tobacco smoke and air pollution, and aging of the population [5]. Main symptoms of COPD are chronic and progressive dyspnea, cough, sputum production, wheezing, and chest tightness, which vary during the day and the week [2]. Other symptoms include fatigue, muscle weakness and weight loss [6, 7]. More specifically, COPD is recognized as a multisystem disease with systemic manifestations, such as skeletal muscle dysfunction, metabolic syndrome, osteoporosis, cardiovascular diseases, diabetes, anemia, symptoms of depression and anxiety [8, 9].

\section{Management of COPD}

Smoking cessation and pharmacologic treatment are usually targeted first to reduce the symptoms of COPD, reduce the frequency and severity of exacerbations, and improve health status and exercise tolerance [10,11]. Pulmonary rehabilitation (PR) is defined as "a comprehensive intervention based on a thorough patient assessment followed by patient tailored therapies that include, but are not limited to, exercise training, education, and behavioral change, designed to improve the physical and psychological condition of people with chronic respiratory disease and to promote the long-term adherence to health-enhancing behaviors". PR has become a core component of the management of COPD [12]. It has been shown to be an effective treatment to improve dyspnea, fatigue, exercise capacity and health related quality of life in patients with COPD [13]. In addition, risk of hospitalization, mortality and healthcare utilization costs are reduced in patients 
with COPD following PR [12, 14].

\section{Functional limitations in patients with COPD}

Patients with COPD demonstrate functional limitations, which are decrements of basic physical or mental actions (e.g. mobility, lower extremity functioning, exercise performance and cognitive function) $[15,16]$. Patients with COPD are characterized by reduced activities in daily life compared to healthy individuals $[17,18]$. Not surprisingly, patients with COPD have an impaired exercise capacity as compared to healthy individuals [1921]. The complex interaction between symptoms, ventilatory impairment, gas exchange limitations, and skeletal muscle dysfunction may cause exercise intolerance in COPD [22]. The main contributor to exercise intolerance is ventilatory limitation. Mostly due to an imbalance between ventilator demand and ventilatory capacity [23]. In addition, dynamic hyperinflation, defined as a temporary increase in operating lung volumes above their resting value, results in the inability to sufficiently emptying the lung. In turn, this imposes constrains on tidal volume, and contributes to impaired exercise capacity and the increased sensation of dyspnea [24].

Lower-limb muscle dysfunction contributes to exercise intolerance in patients with COPD [25] and may be attributable to inactivity-induced deconditioning, systemic inflammation, oxidative stress, smoking, blood gas disturbances, malnutrition, low anabolic hormone levels, aging and corticosteroid use [26, 27]. Consequently, this may lead to muscle alterations, such as mitochondrial dysfunction, a shift from slow-twitch (type I) aerobic towards fast-twitch (type II) anaerobic muscle fibers, a reduction in capillaries per muscle fiber and a loss of muscle mass. These alterations lead to muscle weakness and reduced endurance. Reduced exercise capacity often leads to reduced physical activity with disease progression, resulting in aggravated muscle dysfunction and increase in symptoms, such as fatigue and dyspnea [28]. Consequently, patients with COPD adapt or reduce activities in daily life, become more disable, which in turn leads to a reduced quality of life $[29,30]$.

\section{Evaluation of exercise capacity in COPD}

Assessment of exercise capacity in COPD is an important aspect in the evaluation of the impact of disease severity on functional status, the effectiveness of therapeutic interventions and it has prognostic value [20,31,32]. Field exercise tests, such as the 6-minute 
walk test (6MWT), are commonly used to evaluate exercise capacity in COPD [20]. This self-paced walk test has strong associations with measures of maximal exercise performance and physical activity [20]. The 6MWT is well tolerated [21] and has proven to be a reliable clinical instrument in providing reproducible data, which are used to detect and quantify changes in exercise capacity after an intervention. In addition, this test has been considered as a useful tool to prescribe high-intensity exercise in patients with COPD [33]. Furthermore, evidence has showed that a reduced 6-minute walk distance is associated with increased mortality and hospitalization in patients with COPD [32].

\section{Gait in patients with COPD}

Patients with COPD have reported gait (walking) as one of the most problematic activity in daily life [34]. To date, it is well known that patients with COPD have a reduced gait speed as compared to healthy individuals [35, 36]. Gait speed may be considered as a general measure of overall functional status in COPD [37], and could be used to predict survival and risk of hospitalization [20]. However, studies examining gait more in detail among patients with COPD are limited. The first investigation in this area, a retrospective study by Yentes et al. [38] observed an association between COPD severity and gait abnormalities, such as the presence of limping and shuffling. Subsequent studies, reported increased stride time, reduced cadence and stride length in patients with COPD as compared to healthy older adults [35, 39-42]. Lahousse et al. [39] demonstrated that cadence and speed were positively related with the degree of airflow limitation. More specifically, reduced cadence and speed were found in those who fell in the previous year as compared to non-fallers, suggesting a possible link between gait impairments and fall risk in COPD. In addition, a trade-off between the ventilatory limitations and balance control during walking in COPD is possibly associated with alterations in gait characteristics.

Mediolateral trunk accelerations were increased in patients with COPD during the 6MWT, indicating that adjustment of step width was necessary to compensate for balance disturbances during the test [35]. In addition, reduced step width standard deviation, reflecting the amount of stride-to-stride fluctuations, was found in patients with COPD [42]. Though these findings may seem contradictory to each other, they are complementary as both findings support the idea of a general inability to compensate for instability, and thereby predisposing the patient to a fall [43]. Both increases and decreases in stride-to-stride fluctuations are associated with fall history in older adults [44, 45]. Moreover, falls are a major health concern, since falling is the main cause of pain, inactivity, disability, loss of functional dependence, mortality, hospitalization and decreased quality of life in older adults. The causes of falls are multifactorial, however, most of them happen during walk- 
ing [46]. Therefore, impaired gait function is one of the main risk of falls [47]. Impaired gait function may be associated with balance deficiencies and increased fall risk reported in patients with COPD [18, 48-51]. For instance, a study by Yentes et al. [52] reported increased peak power absorption during midstance in the ankle and a lack of increase in peak ankle dorsiflexion moment under fatigued condition. These results indicate difficulties in controlling the foot shortly after heel strike and therefore tibialis anterior weakness in patients with COPD. McCamley et al. [53] reported that gait deficits did not seem to be apparent in patients with COPD prior to the onset of fatigue. The evidence in gait characteristics in patients with COPD is limited and different factors may be associated with gait impairments, such as fall risk. Moreover, basic activities of daily living, such as walking, are essential to a patient's independence [54]. Therefore, insight into gait impairments in patients with COPD is necessary and will be addressed in the current thesis.

\section{Gait assessment}

Gait is one of the most common activities in daily life [55]. It involves the neural and musculoskeletal system as well as the cardiorespiratory system. When modifications to normal locomotion are demanded as a result of dysfunctional neuromusculoskeletal or other pathological states, adaptations may be demonstrated [56]. For example, step length is often asymmetric in prosthetic gait [57] and walking speed decreases in patients with peripheral arterial disease [58].

Three-dimensional (3D) gait analysis has proven to be a precise and quantitative method to identify an individual's ability to walk. It enables insight into the locomotor system's behavior, the capacity to identify the severity or nature of a locomotor disease or injury, and to evaluate the effect of a treatment [59]. 3D gait analysis is considered the gold standard for gait evaluation in patients with gait abnormalities. Optical 3D motion capture systems may use a series of infrared cameras to track the position of retroreflective markers placed on bony landmarks of the subject's body according to a biomechanical model. It is often used to quantify spatiotemporal (distance and time), kinematic (motion of bodies), kinetic (the forces acting on bodies) parameters using marker trajectories and force plates data.

Instrumented treadmills are increasingly applied in gait analysis. Treadmill based gait analysis offers some advantages over overground gait analysis, such as an increased number of gait cycles that are recorded consecutively and it requires less laboratory space [60]. The Gait Real-time Analysis Interactive Lab (GRAIL, Motek Medical B.V., Amsterdam, the Netherlands) comprises of a 3D motion analysis system with a dual-belt, in- 
strumented treadmill placed in a virtual reality environment with 180 degrees projection. Optic flow of the virtual reality environment is synchronized with the treadmill velocity. Moreover, virtual reality in such a setting has been reported as more similar to overground walking [60]. The GRAIL enables self-paced treadmill walking via a feedback-controlled algorithm, providing fluctuations in walking speed that are comparable to overground walking [61].

\section{Stride-to-stride fluctuations in gait}

Gait patterns from healthy individuals seem to remain relatively constant over consecutive gait cycles, even during unconstrained walking. However, closer and more careful examination reveals stride-to-stride fluctuations in gait patterns over time [62-64]. These fluctuations in gait are defined as the normal variations that occur in motor performance across multiple repetitions of a task [65]. As Bernstein [66] described, multiple degrees of freedom of the body, including joints, muscles and the nervous system, combine with external forces during movement to produce numerous patterns, forms and strategies. The redundancy of the system allows for the use of multiple strategies to accomplish any given task. These fluctuations were traditionally neglected and considered as noise in the locomotor system [67]. However, movement variability has been identified as an inherent feature of the human locomotor system [59] and may reflect the capability of the locomotor system to make flexible adaptations during walking [68, 69]. Stride-to-stride fluctuations provide information about health status and can be related to age [70, 71], disease [42, 67, 72-74], and the risk of falling [75, 76]. A deterioration of these temporal fluctuations, e.g. both periodic and random fluctuations, may represent a decline in the healthy flexibility that is associated with rigidity and inability to adapt, such as those associated with unhealthy states [77]. These stride-to-stride fluctuations can be assessed using a 3D motion analysis, such as the GRAIL system.

\section{Improving gait performance in COPD}

Skeletal muscle dysfunction, one of the extra-pulmonary manifestations in COPD, contributes to functional limitations [25, 78]. Therefore, skeletal muscles are a potential site to improve patients' level of functional mobility. Exercise training is an important component in PR and is effective in improving skeletal muscle function and exercise tolerance in patients with COPD [79, 80]. Exercise training alters muscle fiber morphology and structural characteristics, resulting in increased muscle strength and endurance in patients 
with COPD [81]. More specifically, fiber type distribution reverses in favor to muscle fiber type I, and increases are found in cross-sectional area, the activity of oxidative enzymes of the muscle fiber, and capillary density in patients with COPD [82-86]. These changes in the locomotor system may contribute to an improved gait function in COPD.

Exercise training can be administered using different training modalities. However, the combination of constant-load, interval and strength training improves exercise capacity and muscle strength [87], to a greater degree than either strategy alone in patients with COPD [88]. However, there is a need to identify whether and to what extent the improvements in skeletal muscle function are related to gait function in COPD. Furthermore, muscle strength is a key contributor to balance problems and falls in older adults $[89,90]$ and in patients with COPD [48, 91, 92]. As most falls occur during walking [93-97], gait and balance training might be of interest in improving gait function and balance control in COPD, reducing the risk of a fall.

\section{Objective of this thesis}

Given the decreased health related outcomes due to a reduced exercise capacity and limited ability to walk in patients with COPD, it is of importance to increase our understanding of gait characteristics in patients with COPD. Therefore, the main objective of this thesis was to evaluate gait characteristics in patients with COPD. To achieve this aim, three research questions were examined:

- Is the 6-minute walk test performed on a self-paced treadmill, a valid method to assess the 6-minute walk distance as compared to the overground condition in patients with COPD? (Chapter 2)

- Do patients with COPD demonstrate alterations in gait characteristics as compared to non-COPD subjects? (Chapter 3 - Chapter 5)

- Does the current pulmonary rehabilitation program improve gait characteristics in patients with COPD? (Chapter 6)

\section{Outline of this thesis}

Chapter 2 provides more insight in the use of the 6-minute walk test performed on the Gait Real-time Analysis Interactive Lab in patients with COPD and non-COPD subjects. 
Chapter 3 describes the spatiotemporal gait characteristics in patients with COPD during the Gait Real-time Analysis Interactive Lab-based 6-minute walk test.

Chapter 4 determines the stride-to-stride fluctuations in patients with COPD during the Gait Real-time Analysis Interactive Lab-based 6-minute walk test.

Chapter $\mathbf{5}$ explores the stride-to-stride fluctuations in the lower extremity of patients with COPD while walking at different speeds.

Chapter 6 describes the effects of pulmonary rehabilitation on gait characteristics in patients with COPD.

Chapter 7 discusses the main results described in previous chapters and recommendations for future research and clinical practice. 


\section{Bibliography}

1. Global strategy for the diagnosis, management, and prevention of COPD Web Page. Accessed: 27-072018. http: //goldcopd.org/ (Global Initiative for Chronic Obstructive Lung Disease).

2. Vogelmeier, C. F. et al. Global Strategy for the Diagnosis, Management, and Prevention of Chronic Obstructive Lung Disease 2017 Report: GOLD Executive Summary. Arch Bronconeumol 53, 128-149 (2017).

3. Lozano, R. et al. Global and regional mortality from 235 causes of death for 20 age groups in 1990 and 2010: a systematic analysis for the Global Burden of Disease Study 2010. Lancet 380, 2095-128 (2012).

4. Chronic obstructive pulmonary disease (COPD) Web Page. Accessed: 01-01-2018. http : //www . who . int/respiratory/copd/en/ (World Health Organization).

5. Mathers, C. D. \& Loncar, D. Projections of global mortality and burden of disease from 2002 to 2030. PLoS Med 3, e442 (2006).

6. Janssen, D. J. et al. Symptoms, comorbidities, and health care in advanced chronic obstructive pulmonary disease or chronic heart failure. J Palliat Med 14, 735-43 (2011).

7. Schols, A. M. et al. Prevalence and characteristics of nutritional depletion in patients with stable COPD eligible for pulmonary rehabilitation. Am Rev Respir Dis 147, 1151-6 (1993).

8. Barnes, P. J. \& Celli, B. R. Systemic manifestations and comorbidities of COPD. Eur Respir J 33, 1165-85 (2009).

9. Fabbri, L. M. \& Rabe, K. F. From COPD to chronic systemic inflammatory syndrome? Lancet 370, 797-9 (2007).

10. Celli, B. R. et al. Effect of pharmacotherapy on rate of decline of lung function in chronic obstructive pulmonary disease: results from the TORCH study. Am J Respir Crit Care Med 178, 332-8 (2008).

11. Van Eerd, E. A. et al. Smoking cessation for people with chronic obstructive pulmonary disease. Cochrane Database Syst Rev, CD010744 (2016).

12. Spruit, M. A. et al. An official American Thoracic Society/European Respiratory Society statement: key concepts and advances in pulmonary rehabilitation. Am J Respir Crit Care Med 188, e13-64 (2013).

13. McCarthy, B. et al. Pulmonary rehabilitation for chronic obstructive pulmonary disease. Cochrane Database Syst Rev, CD003793 (2015).

14. Puhan, M. A. et al. Pulmonary rehabilitation following exacerbations of chronic obstructive pulmonary disease. Cochrane Database Syst Rev 12, CD005305 (2016).

15. Eisner, M. D. et al. COPD as a systemic disease: impact on physical functional limitations. Am J Med 121, 789-96 (2008).

16. Mador, M. J. \& Bozkanat, E. Skeletal muscle dysfunction in chronic obstructive pulmonary disease. Respir Res 2, 216-24 (2001).

17. Lahaije, A. J. et al. Physiologic limitations during daily life activities in COPD patients. Respir Med 104, 1152-9 (2010).

18. Roig, M. et al. Deficits in muscle strength, mass, quality, and mobility in people with chronic obstructive pulmonary disease. J Cardiopulm Rehabil Prev 31, 120-4 (2011).

19. Hamilton, A. L. et al. Symptom intensity and subjective limitation to exercise in patients with cardiorespiratory disorders. Chest 110, 1255-63 (1996).

20. Holland, A. E. et al. An official European Respiratory Society/American Thoracic Society technical standard: field walking tests in chronic respiratory disease. Eur Respir J 44, 1428-46 (2014).

21. Singh, S. J. et al. An official systematic review of the European Respiratory Society/American Thoracic Society: measurement properties of field walking tests in chronic respiratory disease. Eur Respir J 44, 1447-78 (2014).

22. Pepin, V. et al. Exercise capacity in chronic obstructive pulmonary disease: mechanisms of limitation. COPD 4, 195-204 (2007).

23. Vogiatzis, I., Zakynthinos, G. \& Andrianopoulos, V. Mechanisms of physical activity limitation in chronic lung diseases. Pulm Med 2012, 634761 (2012). 
24. O'Donnell, D. E. \& Webb, K. A. The major limitation to exercise performance in COPD is dynamic hyperinflation. J Appl Physiol (1985) 105, 753-5, 753-5 (2008).

25. Gosselink, R., Troosters, T. \& Decramer, M. Peripheral muscle weakness contributes to exercise limitation in COPD. Am J Respir Crit Care Med 153, 976-80 (1996).

26. Kim, H. C., Mofarrahi, M. \& Hussain, S. N. Skeletal muscle dysfunction in patients with chronic obstructive pulmonary disease. Int J Chron Obstruct Pulmon Dis 3, 637-58 (2008).

27. Maltais, F. et al. An official American Thoracic Society/European Respiratory Society statement: update on limb muscle dysfunction in chronic obstructive pulmonary disease. Am J Respir Crit Care Med 189, e15-62 (2014).

28. Polkey, M. I. \& Moxham, J. Attacking the disease spiral in chronic obstructive pulmonary disease. Clin Med (Lond) 6, 190-6 (2006).

29. Martinez, F. J. et al. Predictors of mortality in patients with emphysema and severe airflow obstruction. Am J Respir Crit Care Med 173, 1326-34 (2006).

30. Oga, T. et al. Analysis of the factors related to mortality in chronic obstructive pulmonary disease: role of exercise capacity and health status. Am J Respir Crit Care Med 167, 544-9 (2003).

31. Andrianopoulos, V. et al. Prognostic value of variables derived from the six-minute walk test in patients with COPD: Results from the ECLIPSE study. Respir Med 109, 1138-46 (2015).

32. Spruit, M. A. et al. Predicting outcomes from 6-minute walk distance in chronic obstructive pulmonary disease. J Am Med Dir Assoc 13, 291-7 (2012).

33. Rodrigues, A. et al. Is the six-minute walk test a useful tool to prescribe high-intensity exercise in patients with chronic obstructive pulmonary disease? Heart Lung 45, 550-556 (2016).

34. Annegarn, J. et al. Problematic activities of daily life are weakly associated with clinical characteristics in COPD. J Am Med Dir Assoc 13, 284-90 (2012).

35. Annegarn, J. et al. Differences in walking pattern during 6-min walk test between patients with COPD and healthy subjects. PLoS One 7, e37329 (2012).

36. Ilgin, D. et al. Gait speed as a functional capacity indicator in patients with chronic obstructive pulmonary disease. Ann Thorac Med 6, 141-6 (2011).

37. Karpman, C. \& Benzo, R. Gait speed as a measure of functional status in COPD patients. Int J Chron Obstruct Pulmon Dis 9, 1315-20 (2014).

38. Yentes, J. M. et al. Walking abnormalities are associated with COPD: An investigation of the NHANES III dataset. Respir Med 105, 80-7 (2011).

39. Lahousse, L. et al. Gait patterns in COPD: the Rotterdam Study. Eur Respir J 46, 88-95 (2015).

40. Nantsupawat, N. et al. Gait Characteristics in Patients With Chronic Obstructive Pulmonary Disease. J Prim Care Community Health 6, 222-6 (2015).

41. Rutkowski, S. et al. Gait analysis of patients with chronic obstructive pulmonary disease 24-33 (2014).

42. Yentes, J. M. et al. Patients with Chronic Obstructive Pulmonary Disease Walk with Altered Step Time and Step Width Variability as Compared with Healthy Control Subjects. Ann Am Thorac Soc 14, 858-866 (2017).

43. Maki, B. E. Gait changes in older adults: predictors of falls or indicators of fear. J Am Geriatr Soc 45, 313-20 (1997).

44. Beauchet, O. et al. Gait variability among healthy adults: low and high stride-to-stride variability are both a reflection of gait stability. Gerontology 55, 702-6 (2009).

45. Brach, J. S. et al. Too much or too little step width variability is associated with a fall history in older persons who walk at or near normal gait speed. J Neuroeng Rehabil 2, 21 (2005).

46. Bridenbaugh, S. A. \& Kressig, R. W. Laboratory review: the role of gait analysis in seniors' mobility and fall prevention. Gerontology 57, 256-64 (2011).

47. Verghese, J. et al. Quantitative gait markers and incident fall risk in older adults. J Gerontol A Biol Sci Med Sci 64, 896-901 (2009). 
48. Beauchamp, M. K. et al. Impairments in systems underlying control of balance in COPD. Chest 141, 1496503 (2012).

49. Hellstrom, K. et al. Fear of falling, fall-related self-efficacy, anxiety and depression in individuals with chronic obstructive pulmonary disease. Clin Rehabil 23, 1136-44 (2009).

50. Oliveira, C. C. et al. Falls by individuals with chronic obstructive pulmonary disease: a preliminary 12month prospective cohort study. Respirology 20, 1096-101 (2015).

51. Smith, M. D. et al. Balance is impaired in people with chronic obstructive pulmonary disease. Gait \& Posture 31, 456-60 (2010).

52. Yentes, J. M. et al. Gait mechanics in patients with chronic obstructive pulmonary disease. Respir Res 16, 31 (2015).

53. McCamley, J. D. et al. Gait deficiencies associated with peripheral artery disease are different than chronic obstructive pulmonary disease. Gait Posture 57, 258-264 (2017).

54. Ozsoy, I. et al. Factors Influencing Activities of Daily Living in Subjects With COPD. Respir Care 64, 189195 (2019).

55. Pirker, W. \& Katzenschlager, R. Gait disorders in adults and the elderly : A clinical guide. Wien Klin Wochenschr 129, 81-95 (2017).

56. Chaitow, L. \& DeLany, J. in Clinical Application of Neuromuscular Techniques, Volume 2 (Second Edition) (eds Chaitow, L. \& DeLany, J.) 61-84 (Churchill Livingstone, Oxford, 2011).

57. Dingwell, J. B., Davis, B. L. \& Frazier, D. M. Use of an instrumented treadmill for real-time gait symmetry evaluation and feedback in normal and trans-tibial amputee subjects. Prosthet Orthot Int 20, 101-10 (1996).

58. Gardner, A. W., Forrester, L. \& Smith, G. V. Altered gait profile in subjects with peripheral arterial disease. Vasc Med 6, 31-4 (2001).

59. Horst, F. et al. Intra-individual gait patterns across different time-scales as revealed by means of a supervised learning model using kernel-based discriminant regression. PLoS One 12, e0179738 (2017).

60. Sloot, L. H., van der Krogt, M. M. \& Harlaar, J. Effects of adding a virtual reality environment to different modes of treadmill walking. Gait \& Posture 39, 939-45 (2014).

61. Sloot, L. H., van der Krogt, M. M. \& Harlaar, J. Self-paced versus fixed speed treadmill walking. Gait \& Posture 39, 478-84 (2014).

62. Dingwell, J. B. et al. Slower speeds in patients with diabetic neuropathy lead to improved local dynamic stability of continuous overground walking. J Biomech 33, 1269-77 (2000).

63. Hausdorff, J. M. et al. Is walking a random walk? Evidence for long-range correlations in stride interval of human gait. J Appl Physiol (1985) 78, 349-58 (1995).

64. Stergiou, N. Nonlinear Analysis for Human Movement Variability (CRC Press, Boca Raton, 2016).

65. Stergiou, N. \& Decker, L. M. Human movement variability, nonlinear dynamics, and pathology: is there a connection? Hum Mov Sci 30, 869-88 (2011).

66. Bernstein, N. The Coordination and Regulation of Movements (Pergamon Press, London, 1967).

67. Dingwell, J. B. \& Cusumano, J. P. Nonlinear time series analysis of normal and pathological human walking. Chaos 10, 848-863 (2000).

68. Lipsitz, L. A. Dynamics of stability: the physiologic basis of functional health and frailty. J Gerontol A Biol Sci Med Sci 57, B115-25 (2002).

69. Lipsitz, L. A. \& Goldberger, A. L. Loss of 'complexity' and aging. Potential applications of fractals and chaos theory to senescence. JAMA 267, 1806-9 (1992).

70. Buzzi, U. H. et al. Nonlinear dynamics indicates aging affects variability during gait. Clin Biomech (Bristol, Avon) 18, 435-43 (2003).

71. Kang, H. G. \& Dingwell, J. B. Effects of walking speed, strength and range of motion on gait stability in healthy older adults. J Biomech 41, 2899-905 (2008). 
72. Harbourne, R. T. \& Stergiou, N. Movement variability and the use of nonlinear tools: principles to guide physical therapist practice. Phys Ther 89, 267-82 (2009).

73. Hausdorff, J. M. Gait dynamics in Parkinson's disease: common and distinct behavior among stride length, gait variability, and fractal-like scaling. Chaos 19, 026113 (2009).

74. Myers, S. A. et al. Gait variability is altered in patients with peripheral arterial disease. J Vasc Surg 49, 924-931 e1 (2009).

75. Hausdorff, J. M., Rios, D. A. \& Edelberg, H. K. Gait variability and fall risk in community-living older adults: a 1-year prospective study. Arch Phys Med Rehabil 82, 1050-6 (2001).

76. Lockhart, T. E. \& Liu, J. Differentiating fall-prone and healthy adults using local dynamic stability. Ergonomics 51, 1860-72 (2008).

77. Decker, L., Cignetti, F. \& Stergiou, N. Complexity of Human Gait. Rev Andal Med Deporte 3, 2-12 (2010).

78. Man, W. D. et al. Exercise and muscle dysfunction in COPD: implications for pulmonary rehabilitation. Clin Sci (Lond) 117, 281-91 (2009).

79. De Brandt, J. et al. Changes in lower limb muscle function and muscle mass following exercise-based interventions in patients with chronic obstructive pulmonary disease: A review of the English-language literature. Chron Respir Dis 15, 182-219 (2018).

80. Nyberg, A. et al. Adaptations in limb muscle function following pulmonary rehabilitation in patients with COPD - a review. Rev Port Pneumol (2006) 22, 342-350 (2016).

81. Nici, L. et al. American Thoracic Society/European Respiratory Society statement on pulmonary rehabilitation. Am J Respir Crit Care Med 173, 1390-413 (2006).

82. McKeough, Z. J. et al. Exercise capacity and quadriceps muscle metabolism following training in subjects with COPD. Respir Med 100, 1817-25 (2006).

83. Vogiatzis, I. et al. Effect of pulmonary rehabilitation on muscle remodelling in cachectic patients with COPD. Eur Respir J 36, 301-10 (2010).

84. Vogiatzis, I. et al. Effects of rehabilitative exercise on peripheral muscle TNFalpha, IL-6, IGF-I and MyoD expression in patients with COPD. Thorax 62, 950-6 (2007)

85. Vogiatzis, I. et al. Skeletal muscle adaptations to interval training in patients with advanced COPD. Chest 128, 3838-45 (2005).

86. Vogiatzis, I. et al. Effect of pulmonary rehabilitation on peripheral muscle fiber remodeling in patients with COPD in GOLD stages II to IV. Chest 140, 744-752 (2011).

87. Bernard, S. et al. Aerobic and strength training in patients with chronic obstructive pulmonary disease. Am J Respir Crit Care Med 159, 896-901 (1999).

88. Ortega, F. et al. Comparison of effects of strength and endurance training in patients with chronic obstructive pulmonary disease. Am J Respir Crit Care Med 166, 669-74 (2002).

89. Maki, B. E. \& Mcllroy, W. E. Postural control in the older adult. Clin Geriatr Med 12, 635-58 (1996).

90. Moreland, J. D. et al. Muscle weakness and falls in older adults: a systematic review and meta-analysis. J Am Geriatr Soc 52, 1121-9 (2004).

91. Beauchamp, M. K., Brooks, D. \& Goldstein, R. S. Deficits in postural control in individuals with COPD emerging evidence for an important secondary impairment. Multidiscip Respir Med 5, 417-21 (2010).

92. Roig, M. et al. Falls in people with chronic obstructive pulmonary disease: an observational cohort study. Respir Med 105, 461-9 (2011).

93. Berg, W. P. et al. Circumstances and consequences of falls in independent community-dwelling older adults. Age Ageing 26, 261-8 (1997).

94. Kelsey, J. L. et al. Heterogeneity of falls among older adults: implications for public health prevention. Am J Public Health 102, 2149-56 (2012).

95. Li, W. et al. Outdoor falls among middle-aged and older adults: a neglected public health problem. Am J Public Health 96, 1192-200 (2006). 
96. Robinovitch, S. N. et al. Video capture of the circumstances of falls in elderly people residing in long-term care: an observational study. Lancet 381, 47-54 (2013).

97. Tinetti, M. E., Speechley, M. \& Ginter, S. F. Risk factors for falls among elderly persons living in the community. N Engl J Med 319, 1701-7 (1988). 



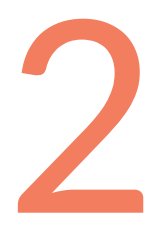

\section{Reproducibility and validity of the} 6-minute walk test using the Gait Real-time Analysis Interactive Lab in patients with COPD and healthy elderly

Wai-Yan Liu, Kenneth Meijer, Jeannet M. Delbressine, Paul J. Willems, Frits M.E. Franssen, Emiel F.M. Wouters, Martijn A. Spruit.

2016. PLOS ONE 11(9): e0162444 


\section{Abstract}

Background and aim The 6-minute walk test (6MWT) in a regular hallway is commonly used to assess functional exercise capacity in patients with chronic obstructive pulmonary disease (COPD). However, treadmill walking might provide additional advantages over overground walking, especially if virtual reality and self-paced treadmill walking are combined. Therefore, this study aimed to assess the reproducibility and validity of the 6MWT using the Gait Real-time Analysis Interactive Lab (GRAIL) in patients with COPD and healthy elderly.

Methods Sixty-one patients with COPD and 48 healthy elderly performed two 6MWTs on the GRAIL. Patients performed two overground 6MWTs and healthy elderly performed one overground test. Differences between consecutive 6MWTs and the test conditions (GRAIL vs. overground) were analyzed.

Results Patients walked further in the second overground test $(24.8 \mathrm{~m}, 95 \% \mathrm{Cl} 15.2-34.4$ $\mathrm{m}, p<0.001)$ and in the second GRAIL test $(26.8 \mathrm{~m}, 95 \% \mathrm{Cl} 13.9-39.6 \mathrm{~m})$. Healthy elderly improved their second GRAIL test $(49.6 \mathrm{~m}, 95 \% \mathrm{Cl} 37.0-62.3 \mathrm{~m})$. The GRAIL 6MWT was reproducible (intra-class coefficients $=0.65-0.80$ ). The best GRAIL 6-minute walk distance (6MWD) in patients was shorter than the best overground 6MWD $(-27.3 \pm 49.1 \mathrm{~m}$, $p<0.001)$. Healthy elderly walked further on the GRAIL than in the overground condition (23.6 $\pm 41.4 \mathrm{~m}, p<0.001)$. Validity of the GRAIL 6MWT was assessed and intra-class coefficient values ranging from $0.74-0.77$ were found.

Discussion/Conclusion The GRAIL is a promising system to assess the 6MWD in patients with COPD and healthy elderly. The GRAIL 6MWD seems to be more comparable to the 6MWDs assessed overground than previous studies on treadmills have reported. Furthermore, good construct validity and reproducibility were established in assessing the 6MWD using the GRAIL in patients with COPD and healthy elderly. 


\section{Introduction}

Chronic obstructive pulmonary disease (COPD) is a highly prevalent chronic disease affecting about $10 \%$ of adults above the age of 40 [1]. COPD affects respiratory function of patients and has systemic consequences as well, including peripheral muscle dysfunction and weakness, which contributes to exercise limitation and impaired quality of life [2, 3]. Exercise intolerance is therefore an important clinical feature in patients with COPD. The 6-minute walk test (6MWT) is a method of obtaining the 6-minute walk distance (6MWD) and is used to evaluate functional exercise capacity. Furthermore, the 6MWT is used to assess response to treatment and predicts morbidity and mortality in patients with COPD [4].

According to the European Respiratory Society/American Thoracic Society (ERS/ATS) guidelines, a flat corridor of at least 30 meters is required to perform a 6MWT [4, 5]. However, not all clinical facilities have such spaces. Therefore, treadmill walking tests offer advantages over overground walking tests, as limited space is needed, providing a safe environment without obstructions [5] and subjects do not have to turn, leading to an increase in walking distance [6].

To date, two studies have been conducted in assessing treadmill-based 6MWDs in patients with COPD. Both studies established a significantly greater mean overground 6MWD compared to a regular treadmill-based 6MWT (+102 and $+51 \mathrm{~m}$, respectively) [7, 8]. However, no difference was found between treadmill-based 6MWT and overground-based 6MWT in healthy subjects in three age groups (48-54 years: $25.1 \mathrm{~m}$; 55-64 years: $15.2 \mathrm{~m}$; 65-75 years: $11.2 \mathrm{~m}$ ) [9].

Both COPD studies with the 6MWT have used regular fixed-paced treadmills. Conversely, the use of a self-paced treadmill, a feedback controlled function that adapts treadmill speed to its user, could be beneficial to adjust the walking speed more naturally and resulting in a more natural gait pattern compared to fixed speed treadmill walking [10]. In addition, the use of virtual reality during treadmill walking is becoming increasingly popular in the area of rehabilitation, since a virtual reality provides an engaging environment and induces a real life sensation [11]. The Gait Real-time Analysis Interactive Lab (GRAIL, Motekforce Link, Amsterdam, the Netherlands) system combines self-paced treadmill walking with virtual reality. Moreover, the GRAIL enables 3D motion capture to analyze gait patterns during walk tests. As the reproducibility of the self-paced treadmillbased 6MWT in patients with COPD remains currently unknown, it is necessary to assess the reproducibility of the 6MWT on the GRAIL and to compare the GRAIL 6MWT with the overground 6MWT. The aims of the current study were therefore to examine the reproducibility and validity of the 6MWT on the GRAIL in patients with COPD and healthy 
elderly.

\section{Materials and methods}

\section{Study design and sample}

A cross-sectional observational study was conducted in CIRO, a center of expertise for chronic organ failure located in Horn, the Netherlands. Sixty-one patients with COPD $\left(\mathrm{FEV}_{1} / \mathrm{FVC}<0.7\right)$ were recruited at pre-rehabilitation assessment between February 2014 and June 2015 [12]. Patients with walking aids, chronic oxygen use, orthopedic ailments and/or neuromuscular co-morbidities affecting their walking patterns were excluded, as well as patients with a history of lung cancer, asthma, sarcoidosis, tuberculosis, and/or lung surgery. Forty-eight healthy elderly, aged 40-85 years, were recruited between July 2014 and October 2015. Healthy elderly were ineligible if respiratory or cardiac diseases, neuromuscular and/or orthopedic ailments were present. The study complied with the Declaration of Helsinki and was approved by the Medical research Ethics Committees United (MEC-U) in the Netherlands (NL46880.060.13). Written consent was obtained from all participants.

\section{Assessment of 6MWD}

The GRAIL (Motekforce Link, Amsterdam, the Netherlands) was used to assess the selfpaced treadmill 6MWDs. The GRAIL comprises of a 3D motion analysis system with a dual-belt, instrumented treadmill and a virtual reality 180 degrees projection screen (Figure 2.1). Four retroflective surface markers were positioned on the anterior superior iliac spine and posterior superior iliac spine of the participant. Marker positions were detected using a ten camera VICON motion analysis system $(100 \mathrm{~Hz}$, Oxford Metrics Ltd., Oxford, UK) and automatically labelled in D-flow (Motekforce Link, Amsterdam, the Netherlands) in order to control treadmill speed via self-paced treadmill walking. The virtual hallway environment was synchronized with the treadmill speed. Participants were not allowed to hold onto the handrails and wore a safety harness during each GRAIL 6MWT. All participants performed one familiarization session on the GRAIL (15-20 minutes) prior to the first GRAIL 6MWT. This session comprised of an explanation of the system and the use of the self-paced function of the treadmill. A four-minute familiarization walk on the treadmill was conducted as well in each participant. Therefore, participants could become accustomed to the virtual hallway environment and self-paced treadmill walking. After the familiarization session, patients performed two GRAIL 6MWTs in two days during the pre-rehabilitation assessment and healthy adults performed the GRAIL 6MWTs 
in one day (Figure 2.2). One GRAlL session took 45 minutes. In addition, all participants performed an overground 6MWT in a 125 meter circular hallway, which took 15-20 minutes. Patients performed two overground 6MWTs during pre-rehabilitation assessment. Healthy elderly performed one overground 6MWT after the GRAIL 6MWTs with a resting period of at least 60 minutes. The overground 6MWD in healthy elderly was considered as the best overground walking distance. All 6MWTs were conducted according to the ERS/ATS guidelines [4]. The 6MWD and average walking speed were assessed. Walking speed was continuously assessed in D-flow and averaged over 6 minutes. Borg scores for both dyspnea and fatigue were recorded before and after the 6MWT, as well as the heart rate and transcutaneous oxygen saturation using a pulse oximeter (Nonin, Care Fusion, San Diego, USA). During the GRAIL 6MWT, post heart rate and transcutaneous oxygen saturation were recorded after the subjects stepped down from the treadmill.

\section{Sample size calculation}

Sample size calculation was based on the results of Stevens et al.[8]. Patients with lung diseases (76\% COPD) achieved on average $374 \pm 78$ meters in the overground 6MWT and $323 \pm 119$ meters on a regular treadmill 6 MWT. Using a posteriori sample size calculation with a power of 0.80 , we calculated a sample size of 36 patients. We hypothesized that the difference in 6MWD will be smaller between overground and GRAIL walking in patients with COPD. We therefore included a larger number of subjects in both groups, which were available for this manuscript.

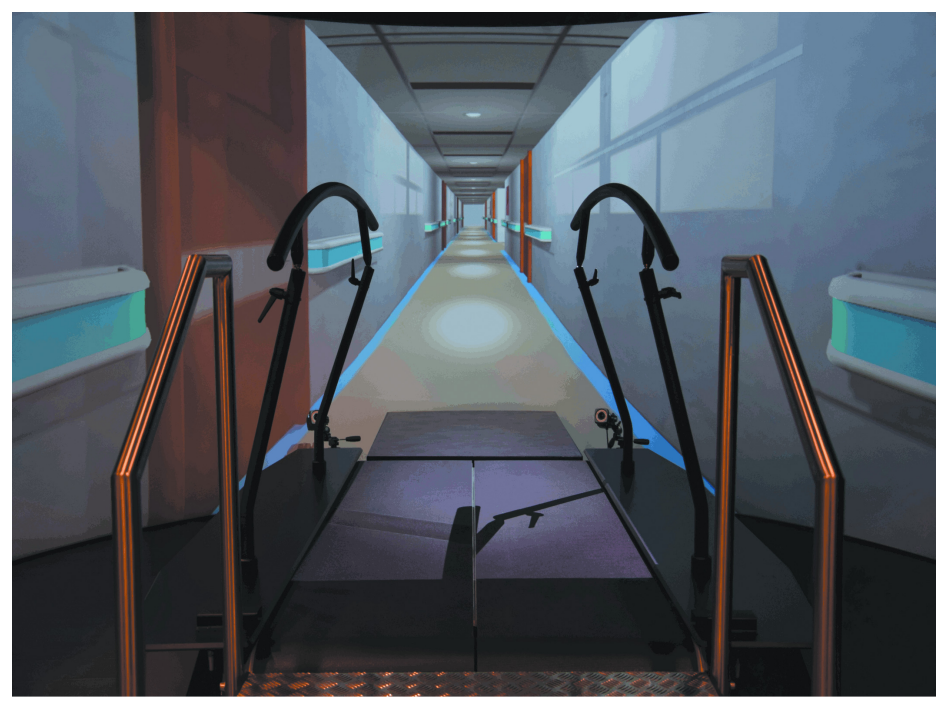

Figure 2.1. The 6MWT of the GRAIL system. 


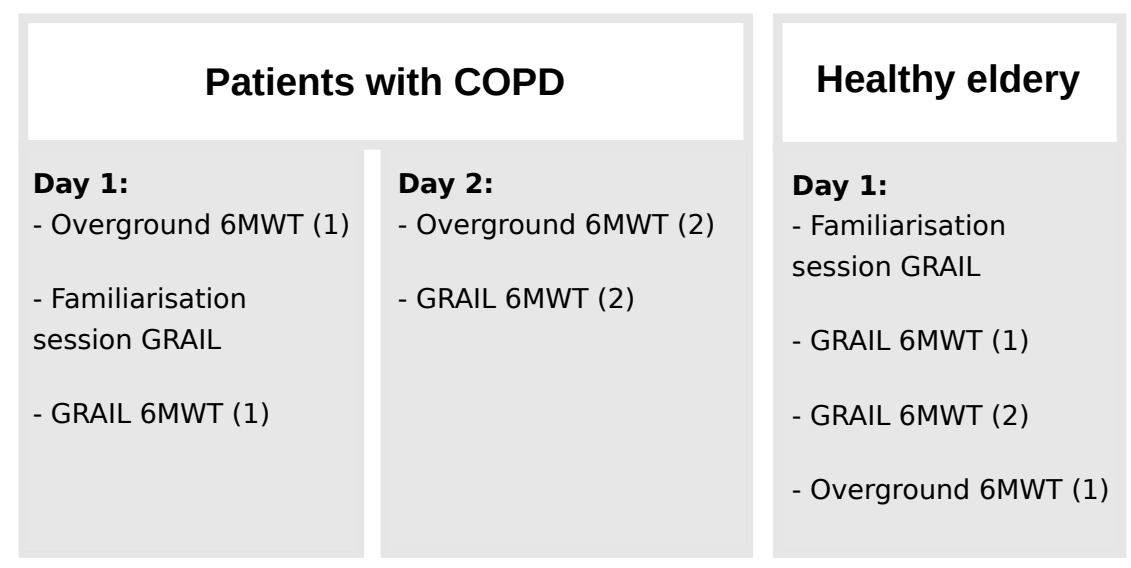

Figure 2.2. Protocol of 6MWT assessment.

\section{Statistical analyses}

The assumption of normally distributed data was checked with the Shapiro-Wilk test. If data were not normally distributed, non-parametric tests were used. Differences between overground 6MWTs in patients, differences between GRAIL 6MWTs in each group and differences between the best GRAIL 6MWT and best overground 6MWT in both groups were identified by paired sample t-tests or two related samples tests. Differences between groups were identified by independent $t$-tests or two independent samples tests. For consistency with previous studies, mean values of non-normally distributed variables are reported. Predicted values of the $6 \mathrm{MWDs}$ for patients and healthy elderly were calculated using the formula of Troosters et al. [13]. The Bland-Altman method was used to assess agreement between the two test conditions. The intra-class correlation coefficient (ICC) values between repetitive GRAIL 6MWTs and between the test conditions were calculated. All analyses were performed using the statistical package SPSS (version 22, IBM SPSS Statistics). Statistical significance was defined as a $p$-value $<0.05$.

\section{Results}

In total 61 patients with COPD and 48 healthy elderly volunteered to participate. Patients had moderate to very severe COPD. Patients and healthy elderly subjects were comparable in age, height, weight and body mass index (BMI). The $\mathrm{FEV}_{1} / \mathrm{FVC}$ and $\mathrm{FEV}_{1} \%$ predicted differed between patients and healthy elderly (Table 2.1). 
Table 2.1. Sample characteristics

\begin{tabular}{lll}
\hline Parameter & $\begin{array}{l}\text { COPD } \\
n=61\end{array}$ & $\begin{array}{l}\text { Healthy } \\
n=48\end{array}$ \\
\hline Age, years & $61.9(6.8)$ & $61.6(6.1)$ \\
Males, \% & 62.3 & 46.8 \\
Height, $\mathrm{m}$ & $1.70(0.09)$ & $1.70(0.09)$ \\
Weight, kg & $78.1(17.7)$ & $77.5(12.8)$ \\
BMI, kg/m ${ }^{2}$ & $27.0(5.1)$ & $26.6(3.2)$ \\
GOLD stage I-IV, $n$ & $9 / 31 / 16 / 5$ & 0 \\
FEV 1 /FVC \% & $41.3(10.6)$ & $77.1(4.5)^{*}$ \\
FEV $1, \%$ predicted & $57.6(20.0)$ & $119.1(16.4)^{*}$ a \\
Non-smoker, $n$ & 1 & 18 \\
Former smoker, $n$ & 56 & 28 \\
Current smoker, $n$ & 4 & 2 \\
\hline
\end{tabular}

Note: Data are presented as number or mean (SD). Non-smoker: $<1$ pack year; Former smoker: quitted smoking before participation at this study with $\geq 1$ pack years; Current smoker: smoked during participation at this study $\geq 1$ pack years. Abbreviations: BMI, body mass index; FEV ${ }_{1}$, forced expiratory volume in $1 \mathrm{~s} ; \mathrm{FVC}$, forced vital capacity.

*: Significant difference between patients with COPD and healthy elderly.

a: Non-parametric test have been used.

\section{Overground 6MWT}

In the overground condition, patients walked $481.4 \mathrm{~m}(95 \% \mathrm{Cl} 465.7-497.0 \mathrm{~m})$ in the first 6MWT and $506.2 \mathrm{~m} \mathrm{(95 \%} \mathrm{Cl} 488.3-524.0 \mathrm{~m})$ in the second 6MWT, with an average increase of $24.8 \mathrm{~m} \mathrm{(95 \%} \mathrm{Cl} \mathrm{15.2-34.4} \mathrm{m;} p<0.001)$. Eighty percent of patients improved during the second overground 6MWT. The best 6MWD in patients was $511.0 \mathrm{~m}(95 \% \mathrm{Cl}$ 494.5-527.6 m), which was $79 \%$ (95\% Cl 76-82\%) of the predicted value. At the end of the best overground 6MWT, patients had Borg dyspnea and fatigue scores of $5.4(95 \% \mathrm{Cl}$ 4.8-6.0) and 5.3 (95\% $\mathrm{Cl} 4.7-5.9)$, respectively. Forty-nine percent of patients desaturated $\left(\mathrm{SpO}_{2}\right.$ nadir $\leq 88 \%$ [14]) during their best overground test. Oxygen saturation decreased by $7.1 \%$ (95\% $\mathrm{Cl} 5.6-8.6 \%$ ) and heart rate increased by $29.5 \mathrm{bpm}(95 \% \mathrm{Cl} 26.4-32.5 \mathrm{bpm})$.

Healthy elderly covered 668.8 m (95\% Cl 647.4-690.1 m), which was 103\% (95\% Cl 100$106 \%$ ) of the predicted value. At the overground 6MWT, healthy elderly had Borg dyspnea and fatigue scores of $1.2(95 \% \mathrm{Cl} 0.9-1.5)$ and 1.2 (95\% Cl 0.9-1.5), respectively. Oxygen saturation decreased by $1.2 \%(95 \% \mathrm{Cl} 0.2-2.2 \%)$ and heart rate increased by $47.3 \mathrm{bpm}$ (95\% $\mathrm{Cl} 42.8-51.9 \mathrm{bpm})$. Difference in best overground 6MWD of patients and healthy elderly was $157.8 \mathrm{~m}$ (95\% Cl 131.5-184.0 m; $p<0.001)$. 
Table 2.2. The GRAIL 6MWTs

\begin{tabular}{|c|c|c|c|c|}
\hline \multirow[b]{2}{*}{ Parameter } & \multicolumn{2}{|c|}{ GRAIL 6MWT (1) } & \multicolumn{2}{|c|}{ GRAIL 6MWT (2) } \\
\hline & COPD & Healthy & COPD & Healthy \\
\hline 6MWD, m & $449.7(89.3)$ & $639.9(77.1)^{\#}$ & $476.4(86.8)^{a^{*}}$ & $689.5(62.2)^{* \#}$ \\
\hline Walking speed, $\mathrm{km} / \mathrm{h}$ & $4.5(0.9)$ & $6.4(0.8)^{\#}$ & $4.8(0.9)^{a^{*}}$ & $6.9(0.7)^{* \#}$ \\
\hline Pre $\mathrm{SpO}_{2}, \%$ & $94.9(1.8)^{\mathrm{ab}}$ & $97.2(1.2)^{\mathrm{a \#}}$ & $95.5(1.6)^{\mathrm{a}^{*}}$ & $97.2(1.0)^{\mathrm{a \#}}$ \\
\hline Post $\mathrm{SpO}_{2}, \%$ & $93.4(4.4)^{\mathrm{a}}$ & $97.4(0.9)^{\mathrm{a} \#}$ & $93.2(4.8)^{\mathrm{a}}$ & $97.2(1.3)^{\mathrm{a \#}}$ \\
\hline$\Delta \mathrm{SpO}_{2}, \%$ & $-1.5(3.9)^{\mathrm{ab} \ddagger}$ & $0.2(1.0)^{\mathrm{a} \ddagger \#}$ & $-2.3(4.3)^{\ddagger^{*}}$ & $0.0(1.0)^{\mathrm{a \#}}$ \\
\hline Pre HR, bpm & $82.4(14.9)^{\mathrm{b}}$ & $65.0(11.1)^{\#}$ & $83.7(14.7)$ & $68.0(12.2)^{* \#}$ \\
\hline Post HR, bpm & $100.9(16.4)$ & $89.3(21.6)^{\#}$ & $102.5(17.9)$ & $99.5(21.1)^{\mathrm{a}^{*}}$ \\
\hline$\Delta \mathrm{HR}, \mathrm{bpm}$ & $18.7(11.0)^{\mathrm{ab} \ddagger}$ & $24.3(15.5)^{\ddagger}$ & $18.8(10.0)^{\text {af }}$ & $31.5(14.2)^{\ddagger^{* \#}}$ \\
\hline Pre dyspnea, score & $1.3(1.1)^{\mathrm{a}}$ & $0.2(0.4)^{\mathrm{a \#}}$ & $1.4(1.2)^{\mathrm{a}}$ & $0.3(0.5)^{\mathrm{a \#}}$ \\
\hline Post dyspnea, score & $4.5(2.0)^{\mathrm{a}}$ & $1.1(1.0)^{\mathrm{a \#}}$ & $4.6(2.3)^{a}$ & $1.2(1.1)^{\mathrm{a} \#}$ \\
\hline$\Delta$ dyspnea, score & $3.2(2.0)^{\ddagger}$ & $0.9(1.0)^{\mathrm{a} \# \#}$ & $3.2(2.2)^{\ddagger}$ & $0.9(0.9)^{\mathrm{a} \# \#}$ \\
\hline Pre fatigue, score & $1.5(1.3)^{\mathrm{a}}$ & $0.3(0.6)^{\mathrm{a \#}}$ & $1.5(1.3)^{\mathrm{a}}$ & $0.3(0.6)^{\mathrm{a \#}}$ \\
\hline Post fatigue, score & $4.3(2.4)^{a}$ & $1.3(1.2)^{\mathrm{a} \#}$ & $4.6(2.3)^{a}$ & $1.3(1.1)^{\mathrm{a} \#}$ \\
\hline$\Delta$ fatigue, score & $2.8(2.3)^{\ddagger}$ & $1.0(1.1)^{\mathrm{a} \# \#}$ & $3.1(2.1)^{\ddagger}$ & $1.0(1.1)^{\mathrm{a} \# \#}$ \\
\hline
\end{tabular}

Note: Data are presented as number or mean (SD). Abbreviations: COPD, chronic obstructive pulmonary disease; 6MWT, 6-minute walk test; 6MWD, 6-minute walk distance; $\mathrm{SpO}_{2}$, pulse oxygen saturation; HR, heart rate; bpm, beats per minute.

‡: Significant difference between pre and post symptoms per trial.

*: Significant difference between the first and second GRAIL 6MWT within each group.

\#: Significant difference between patients with COPD and healthy elderly.

a: Non-parametric test has been used.

b: Missing value in patients with COPD, $n=60$.

\section{GRAIL-based 6MWT}

Patients walked $449.7 \mathrm{~m}(95 \% \mathrm{Cl} 426.8-472.5 \mathrm{~m})$ in the first GRAIL 6MWT and $476.4 \mathrm{~m}$ $(95 \% \mathrm{Cl} 454.2-498.7 \mathrm{~m}$ ) in the second test (Table 2.2), with an average increase of 26.8 $\mathrm{m}$ (95\% Cl 13.9-39.6 m; $p<0.001)$. Seventy-seven percent of all patients performed the second GRAIL test during the pre-rehabilitation assessment. The ICC value of the reproducibility of the GRAIL 6MWTs was 0.80 (95\% $\mathrm{Cl} 0.61-0.89)$ and $75 \%$ of patients improved during the second GRAIL test. The best GRAIL 6MWD in patients was $483.7 \mathrm{~m}(95 \% \mathrm{Cl}$ 462.1-505.3 $\mathrm{m}$ ) (Table 2.3). At the end of the best GRAIL 6MWT, patients had Borg dyspnea and fatigue scores of $4.8(95 \% \mathrm{Cl} 4.2-5.4)$ and $4.6(95 \% \mathrm{Cl} 4.0-5.2)$, respectively. Oxygen saturation decreased by $2.0 \%(95 \% \mathrm{Cl} 0.9-3.13 \%)$ and heart rate increased by 19.1 bpm (95\% Cl 16.4-21.8 bpm). Nine patients (14.8\%) desaturated during their best GRAIL 6MWT. 


\section{Grail versus overground 6MWT}

On average, the best GRAIL-based 6MWD in patients was significantly shorter than the best overground 6MWD $(-27.3 \pm 49.0 \mathrm{~m}, p<0.001)$. Conversely, the GRAIL 6MWD in healthy elderly was significantly greater than the overground 6MWD $(23.6 \pm 41.4 \mathrm{~m}$, $p<0.001$; Table 2.3). The Bland-Altman plot (Figure 2.3) confirms that the majority of patients walked further overground compared to the GRAIL, while the majority of the healthy elderly walked further in the GRAIL condition. Validity of the GRAIL 6MWT compared to overground 6MWT was assessed in both groups, with ICC values of $0.74(95 \% \mathrm{CI}$ $0.51-0.86)$ for patients and $0.77(95 \% \mathrm{Cl} 0.53-0.88)$ for healthy elderly.
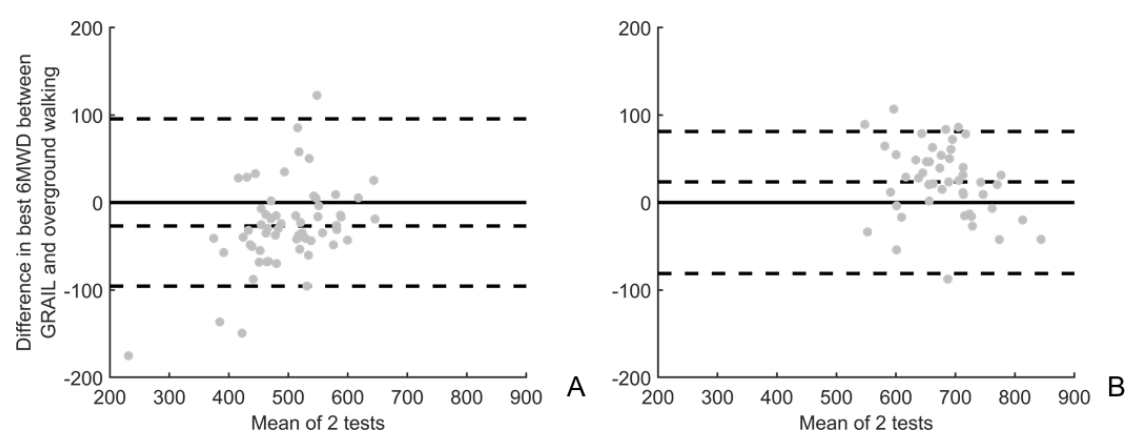

Figure 2.3. Differences between the best overground 6MWT and GRAIL 6MWT in (3A) patients with COPD and (3B) healthy elderly. Bland-Altman plots of the difference between the best overground 6MWT and best GRAIL 6MWT, plotted against the mean value of the two tests in each group. The central solid lines represent the mean difference between the two methods, whereas the lower and upper dashed lines represent the limits of agreement (1.96 SD of mean value).

\section{Discussion}

The present study provides the first evaluation of the reproducibility and validity of the 6MWT assessed by the GRAIL in patients with COPD. It extends previous work on treadmill based $6 \mathrm{MWT}$ by assessing the $6 \mathrm{MWD}$ using virtual reality and self-paced treadmill walking. On average, patients increased their 6MWD between the first and second walk test equally in the overground and GRAIL condition. Furthermore, the 6MWT on the GRAIL showed good reproducibility with ICC values of 0.65 for healthy elderly and 0.80 for patients. The best 6MWD in patients was obtained in overground walking, while healthy elderly covered greater distances using the GRAIL. Moreover, the 6MWT on the GRAIL showed to have good construct validity with ICC values of 0.74 and 0.77 . Therefore, these results indicate that the 6MWD could be reliably and validly assessed by the GRAIL 


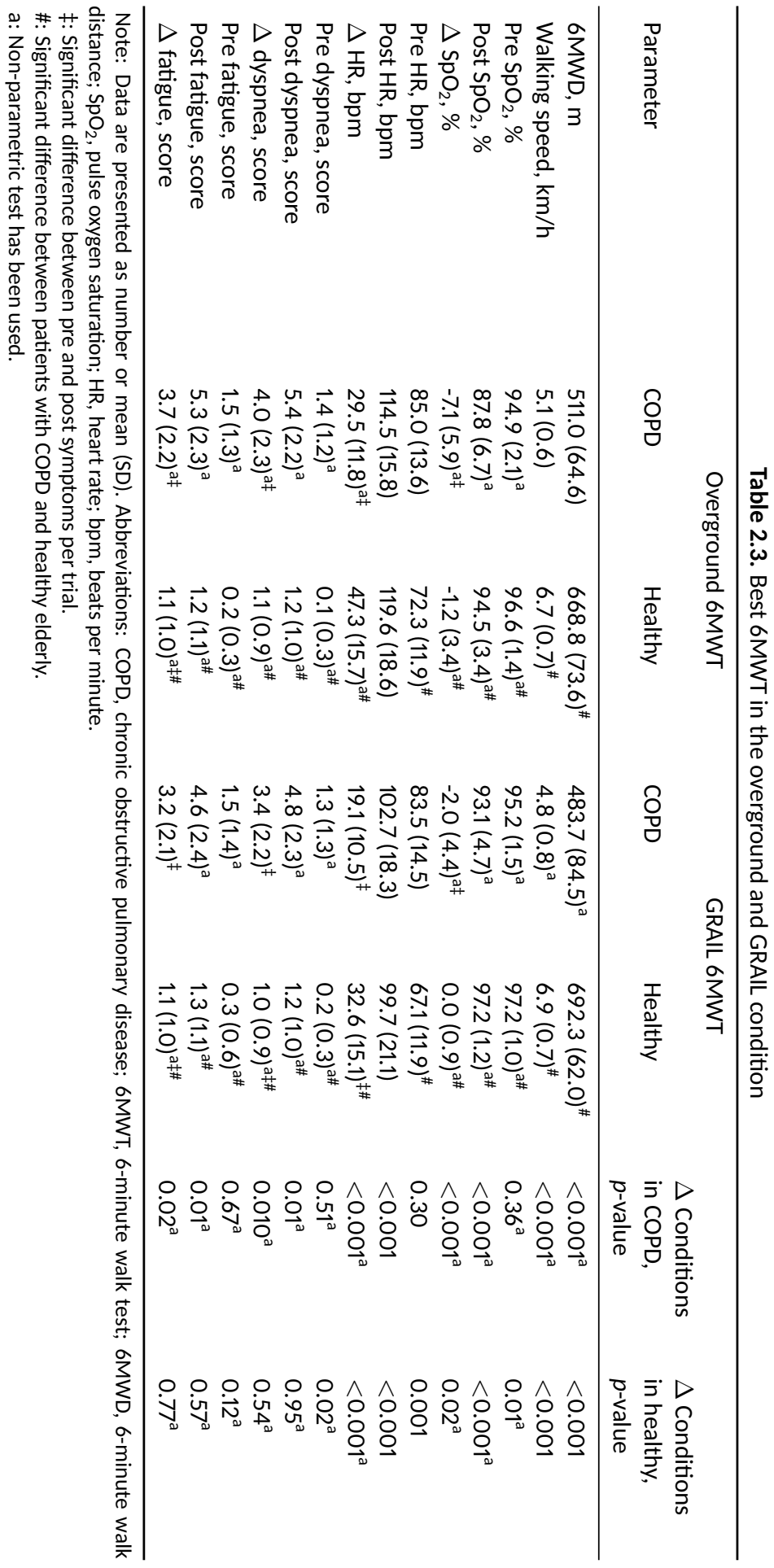


in patients with COPD and healthy elderly.

The 6MWD between the first and second trial improved equally in the overground and GRAIL 6MWT in patients with COPD. These results are similar to existing literature in assessing the $6 \mathrm{MWD}$ within this patient group $[15,16]$. Larger increases in the second GRAIL 6MWT were found in healthy elderly compared to patients, despite all participants undergoing one familiarization session prior to the first GRAIL 6MWT. A possible explanation is that treadmill walking requires different energy expenditure in each of the subject groups [7]. Another possibility could be that self-paced treadmill walking required more effort of the patient than healthy elderly, due to muscle weakness, balance deficits and/or anxiety [17-19]. In addition, adaptability towards learning new tasks might be affected in patients with COPD, as declines in a number of cognitive functions have been observed previously $[20,21]$.

Patients achieved on average a lower 6MWD (-27.5 m) on the GRAIL compared to overground walking. This difference in 6MWD between the test conditions is smaller than previous studies using regular treadmills have established [7, 8]. Our findings are supported by less increase in the Borg dyspnea and fatigue scores, less decrease in oxygen saturation and less increase in heart rate in the GRAIL condition. Healthy elderly, however, achieved greater 6MWDs while walking on the GRAIL, which is in contrast with the findings of Elazzazi et al. [9]. Healthy elderly did not differ in their degree of dyspnea or fatigue between their best overground and best GRAIL test. Therefore, we can assume that healthy elderly experienced equal exertion in performing the 6MWT in each condition. However, this was not seen in the heart rate, as the heart rates were higher after the overground 6MWT compared to the GRAIL 6MWT. Our study showed smaller differences in 6MWD between the test conditions, which might be due to the use of the self-paced treadmill walking. Self-paced treadmill walking offers a more naturally adjustment of walking speed, which could lead to a more natural gait pattern compared to fixed speed treadmill walking [10]. In addition, the overground track in this study required multiple turnarounds compared to the GRAIL condition. However, the turns were larger than most clinical settings use (30 meters). The virtual reality environment could have created a more realistic environment by providing optic flow. By combining the self-paced treadmill walking and virtual reality, a greater 6MWD might have been achieved compared to regular treadmill walking $[11,22]$.

Despite the fact that the familiarization session is only performed prior to the first GRAIL 6MWT, which could have led to less distance covered in the second GRAIL 6MWT, our results indicate that $75 \%$ of patients improved their walk distance during the second GRAIL test. This is comparable to the $80 \%$ of patients who improved during their second overground test. Therefore, we consider this effect to be minimal. Concerning the duration 
of a GRAIL 6MWT. One GRAIL session takes more time to perform compared to the overground 6MWT. In addition, the GRAIL is less accessible to all centers compared to regular treadmills. We do however not argue that the GRAIL should be implemented everywhere to assess the 6MWD only. The GRAIL is a unique method to conduct analysis of gait impairments in patients with COPD, as these patients have reported walking as one of the most problematic activity in daily life [23]. Future study should therefore focus on gait assessment in patients with COPD. Furthermore, predicted distance values and minimal clinical important difference (MCID) of the 6MWT for patients with COPD are available in the overground condition. As these have not been determined in the GRAIL condition, next steps should be to derive new reference values for the GRAIL condition in healthy elderly subjects and to determine MCID, if the GRAIL will be used for further assessment of patients with COPD.

A first limitation of this study is that healthy elderly performed one overground 6MWT, which might have led to a shorter best overground 6MWD. However, a previous study found a minimal difference of 5 meters between two overground 6MWTs in healthy elderly [24]. Therefore, healthy elderly could achieve their best 6MWD during their first overground 6MWT. The second limitation is that there is a time gap between the GRAIL $6 \mathrm{MWT}$ and the post assessment of oxygen saturation levels and heart rates compared to the overground 6MWT. It is possible that conclusions based on the differences in heart rates and oxygen saturation might not explain the differences between the test conditions. A third limitation is that GOLD stage 4 patients are less represented in this study. Therefore, our findings should be carefully interpreted for stage 4 patients. Moreover, complex patients with COPD were excluded, as this is the first study to assess the 6MWT using the GRAIL. Consequently, patients should be able to perform the GRAIL tests without using the handrails and be able to control the self-paced treadmill. A fourth limitation is that this study is a monocentric study, as access to the GRAIL in other centers is currently limited. Moreover, $\mathrm{CIRO}$ is a specialized pulmonary rehabilitation clinic, which may limit the external validity of current findings. A fifth limitation is that the learning effect of 6MWT on the GRAIL in repetitive tests of more than two trials has not been established. Consequently, it is not known if the learning effect attenuates in a third GRAIL 6MWT. Another limitation is that balance during treadmill walking might be affected. However, the GRAIL provides the opportunity to assess quality of gait (e.g. balance) continuously in a virtual reality environment and during self-paced walking, which is not possible using a regular treadmill or in an overground condition. As a result, new insights in determinants of walking balance in patients with COPD could be achieved by using this system.

In conclusion, the GRAIL is a promising system to assess the 6MWD in patients with COPD and healthy elderly. The 6MWD assessed by the GRAIL seems to be more comparable 
to the 6MWDs assessed overground than previous studies on treadmills have reported. Furthermore, good construct validity and reproducibility were established in assessing the 6MWD using the GRAIL in patients with COPD and healthy elderly.

\section{Acknowledgements}

The authors thank the study participants and the staff of CIRO. 


\section{Bibliography}

1. Buist, A. S. et al. International variation in the prevalence of COPD (the BOLD Study): a population-based prevalence study. Lancet 370, 741-50 (2007).

2. Agusti, A. G. et al. Systemic effects of chronic obstructive pulmonary disease. Eur Respir J 21, 347-60 (2003).

3. Wouters, E. F. Chronic obstructive pulmonary disease. 5: systemic effects of COPD. Thorax 57, 1067-70 (2002).

4. Holland, A. E. et al. An official European Respiratory Society/American Thoracic Society technical standard: field walking tests in chronic respiratory disease. Eur Respir J 44, 1428-46 (2014).

5. Society, A. T. ATS statement: guidelines for the six-minute walk test. Am J Respir Crit Care Med 166, 111-7 (2002).

6. Bansal, V. et al. Modifying track layout from straight to circular has a modest effect on the 6-min walk distance. Chest 133, 1155-60 (2008).

7. De Almeida, F. G., Victor, E. G. \& Rizzo, J. A. Hallway versus treadmill 6-minute-walk tests in patients with chronic obstructive pulmonary disease. Respir Care 54, 1712-6 (2009).

8. Stevens, D. et al. Comparison of hallway and treadmill six-minute walk tests. Am J Respir Crit Care Med 160, 1540-3 (1999).

9. Elazzazi, A. et al. Measurement of distance walked and physiologic responses to a 6-minute walk test on level ground and on a treadmill: a comparative study. J Geriatr Phys Ther 35, 2-7 (2012).

10. Sloot, L. H., van der Krogt, M. M. \& Harlaar, J. Self-paced versus fixed speed treadmill walking. Gait \& Posture 39, 478-84 (2014).

11. Sloot, L. H., van der Krogt, M. M. \& Harlaar, J. Effects of adding a virtual reality environment to different modes of treadmill walking. Gait \& Posture 39, 939-45 (2014).

12. Spruit, M. A. et al. Integration of pulmonary rehabilitation in COPD. Lancet 371, 12-3 (2008).

13. Troosters, T., Gosselink, R. \& Decramer, M. Six minute walking distance in healthy elderly subjects. Eur Respir J 14, 270-4 (1999).

14. Andrianopoulos, V. et al. Exercise-induced oxygen desaturation in COPD patients without resting hypoxemia. Respir Physiol Neurobiol 190, 40-6 (2014)

15. Hernandes, N. A. et al. Reproducibility of 6-minute walking test in patients with COPD. Eur Respir J 38, 261-7 (2011).

16. Singh, S. J. et al. An official systematic review of the European Respiratory Society/American Thoracic Society: measurement properties of field walking tests in chronic respiratory disease. Eur Respir J 44, 1447-78 (2014).

17. Bernard, S. et al. Peripheral muscle weakness in patients with chronic obstructive pulmonary disease. Am J Respir Crit Care Med 158, 629-34 (1998).

18. Butcher, S. J., Meshke, J. M. \& Sheppard, M. S. Reductions in functional balance, coordination, and mobility measures among patients with stable chronic obstructive pulmonary disease. J Cardiopulm Rehabil 24, 274-80 (2004).

19. Hellstrom, K. et al. Fear of falling, fall-related self-efficacy, anxiety and depression in individuals with chronic obstructive pulmonary disease. Clin Rehabil 23, 1136-44 (2009).

20. Antonelli-Incalzi, R. et al. Screening of cognitive impairment in chronic obstructive pulmonary disease. Dement Geriatr Cogn Disord 23, 264-70 (2007).

21. Etnier, J. L. \& Berry, M. Fluid intelligence in an older COPD sample after short- or long-term exercise. Med Sci Sports Exerc 33, 1620-8 (2001).

22. Plotnik, M. et al. Self-selected gait speed-over ground versus self-paced treadmill walking, a solution for a paradox. J Neuroeng Rehabil 12, 20 (2015).

23. Annegarn, J. et al. Problematic activities of daily life are weakly associated with clinical characteristics in COPD. J Am Med Dir Assoc 13, 284-90 (2012). 
24. Janaudis-Ferreira, T., Sundelin, G. \& Wadell, K. Comparison of the 6-minute walk distance test performed on a non-motorised treadmill and in a corridor in healthy elderly subjects. Physiotherapy 96, 234-9 (2010). 



\section{CHAPTER}

\section{Spatiotemporal gait characteristics in patients with COPD during the Gait Real-time Analysis Interactive Lab-based 6-minute walk test}

Wai-Yan Liu, Martijn A. Spruit, Jeannet M. Delbressine, Paul J. Willems, Frits M.E. Franssen, Emiel F.M. Wouters, Kenneth Meijer.

2017. PLOS ONE 12(12): e0190099 


\section{Abstract}

Background and aim Overground gait assessment is limited by the analysis of multiple strides or both spatiotemporal gait characteristics, while fixed speed treadmill walking restricts natural gait speed variations. The Gait Real-time Analysis Interactive Lab (GRAIL)based 6-minute walk test (6MWT) enables 3D motion analysis and self-paced treadmill walking, and could provide insight in gait alterations in patients with chronic obstructive pulmonary disease (COPD). The aim of this study is to compare spatiotemporal gait characteristics between patients with COPD and healthy elderly during the GRAIL-based 6MWT.

Materials and methods Eighty COPD patients (60\% male; $62 \pm 7$ years; $\mathrm{FEV}_{1}: 56 \pm 19 \%$ predicted) and 38 healthy elderly ( $63 \%$ male; $62 \pm 6$ years; $\mathrm{FEV}_{1}: 119 \pm 17 \%$ predicted) performed two GRAIL-based 6MWTs. Mean differences and coefficient of variation of spatiotemporal gait characteristics were calculated using the trial with the largest walk distance. Sub-analyses were conducted to account for walking speed differences between groups, and muscle strength and COPD severity within the patient group

Results COPD patients showed increased temporal gait characteristics, decreased stride and step lengths, and increased gait variability compared to healthy elderly $(p<0.01)$. Stride length variability remained increased in COPD after correction for walking speed (MD: $0.98 \%, \mathrm{Cl}: 0.36-1.61, p=0.003$ ). Reduced quadriceps strength did not translate into altered gait characteristics, while COPD severity is associated with swing time (left MD: -0.02s, Cl: -0.04--0.01, p=0.003; right MD: -0.02s, Cl: -0.04-0.01, p=0.003).

Discussion COPD patients performed the GRAIL-based 6MWT differently compared to healthy elderly. Further research should use other variability measures to investigate gait characteristics in COPD, to assess subtle alterations in gait and to enable development of rehabilitation strategies to improve gait, and possibly balance and fall risk in COPD. Other lower limb muscle groups should be considered when investigating gait alterations in COPD.

Conclusion COPD patients have different gait characteristics compared to healthy elderly. Independent of walking speed, COPD patients demonstrate increased stride length variability during the GRAIL-based 6MWT compared to healthy elderly. 


\section{Introduction}

Patients with chronic obstructive pulmonary disease (COPD) report walking as one of the most problematic activity in daily life [1]. Patients with COPD walk less in daily life [2] and achieve shorter walk distances during the 6-minute walk test (6MWT) compared to healthy subjects [3]. Walking distance is an important parameter in the evaluation of treatment and as a prognostic value, in which walking distances $<350 \mathrm{~m}$ on the 6MWT are associated with increased mortality [4]. In addition, patients with COPD demonstrate extra-pulmonary manifestations affecting the muscular system, resulting in muscular dysfunction $[5,6]$ and most probably in gait (walking) alterations.

Gait assessment could provide insight in the biomechanical factors associated with the reduced walk distances in patients with COPD. To date, several studies have explored gait in COPD [3, 7-10]. One study reported that gait alterations, such as limping and shuffling, are associated with disease severity in COPD [11]. Patients with COPD also demonstrate decreased cadence, shorter step lengths, increased time spent in double support and a lack of increase in peak ankle dorsiflexion moment after the onset of breathlessness or leg tiredness compared to healthy subjects, while walking at their comfortable speed [7, $8,10]$. Increased balance disturbances in mediolateral direction in patients with COPD were observed during the 6MWT [3]. Furthermore, patients with COPD walk with an larger step time and smaller step width variability during fixed speed treadmill [12].

Most studies recorded gait in patients with COPD during overground walking, using accelerometry [3], a pressure sensitive mat [7], 2D or 3D motion capture systems [8, 10]. However, the latter three methods can only assess a limited number of consecutive strides, due to space and/or equipment constraints. Gait analysis using instrumented treadmills could be an alternative to overground gait analysis, as they require less laboratory space [13]. However, treadmill walking at fixed speeds restricts subjects to walk with speed variations, resulting in less natural stride variability. Self-paced treadmill walking, involving a feedback-regulated treadmill that allows subjects to walk at their preferred speed, is suggested to be a suitable alternative to fixed speed treadmill walking in gait analysis [13]. In addition, accelerometry enables the recording of multiple strides, but cannot reliably capture the spatial gait characteristics [14].

The Gait Real-time Analysis Interactive Lab (GRAIL) enables self-paced treadmill walking combined with 3D motion capture and could overcome the limitations in overground and fixed speed treadmill walking. Previous studies observed similar spatiotemporal, kinetic and kinematic gait characteristics in self-paced and fixed speed treadmill walking using the GRAIL system $[13,15]$. Moreover, gait speed in self-paced treadmill was comparable to overground walking when using a similar system as the GRAIL [14]. Spatiotemporal gait 
characteristics can therefore be assessed accurately in patients with COPD over multiple consecutive strides and during exercise testing, such as during a 6MWT. As patients with COPD are able to perform the 6MWT using the GRAIL with minimal differences compared to the overground 6MWT [16], additional outcomes can be obtained to identify gait alterations within this population. In addition, as the 6MWT is a submaximal exercise test, challenging the body to walk at speeds outside of the comfortable walking speed can reveal declines in gait or associations in gait characteristics that are otherwise camouflaged at their comfortable walking speed [17].

The aim of the present study is to compare spatiotemporal gait characteristics between patients with COPD and healthy elderly during the GRAIL-based 6MWT (with and without adjustment for expected differences in walking speed; and within the patient sample after stratification for quadriceps muscle strength or COPD severity, described as the degree of airflow limitation). It was hypothesized that patients with COPD would demonstrate alterations in their spatiotemporal gait characteristics as compared to healthy elderly.

\section{Materials and methods}

\section{Study design}

This cross-sectional study was conducted in CIRO, a center of expertise for chronic organ failure in Horn, the Netherlands. Patients with COPD, assessed by spirometry (Carefusion, San Diego, CA, USA) according to the Global Initiative for Chronic Obstructive Lung Disease (GOLD) criteria (post-bronchodilator Forced Expiratory Volume in one second /Forced Vital Capacity; $\mathrm{FEV}_{1} / \mathrm{FVC}$ ratio $<0.70$ ), were recruited at regular pre-rehabilitation assessment. Patients with walking aids, chronic oxygen therapy, orthopedic ailments and/or neuromuscular co-morbidities affecting their walking patterns were excluded, as well as patients with a history of lung cancer, asthma, sarcoidosis, tuberculosis and/or lung surgery. Healthy elderly were recruited amongst healthy subjects who participated in previous trials [18], from co-workers or from healthy relatives of patients. These healthy elderly subjects were included as the non-COPD group (post-bronchodilator $\mathrm{FEV}_{1} / \mathrm{FVC}$ ratio $>0.70$ ), if subjects did not have any self-reported neuromuscular and/or orthopedic ailments. Spirometry and electrocardiography were conducted prior to the GRAIL-based 6MWT. Then, quadriceps muscle strength was measured (Biodex System 4 Pro, Biodex Medical Systems, Inc., New York, USA). Quadriceps muscle strength was calculated as the peak torque of $\%$ predicted, based on Borges et al. [19]. Participants performed thirty volitional maximal contractions at an angular velocity of 90 degrees per second. This study (M13-1374) complied with the Declaration of Helsinki and was approved by the Medical 
research Ethics Committees United (MEC-U) in the Netherlands. This study is registered at the Dutch Trial Register (NTR4421). Written consent was obtained from all participants.

\section{Assessment of gait}

Each participant performed two 6MWT's using the GRAIL (Motekforce Link, Amsterdam, the Netherlands), a 3D motion capture system with an instrumented dual-belt treadmill and virtual reality on a 180 degrees projection screen (Figure 3.1). Optical flow of the virtual reality environment was synchronized with the treadmill velocity. Subjects wore tight fitting shorts. Twenty-five reflective markers were placed on anatomical landmarks of each participant according to the Human Body Model (HBM) of the lower limb (Figure 3.2) [20]. The 3D marker trajectories were collected $(100 \mathrm{~Hz})$ with a ten camera 3D motion capture system (Vicon Nexus, Oxford Metrics Ltd., Oxford, UK) and processed in D-flow (Motekforce Link, Amsterdam, the Netherlands). Ground reaction force data from heel contact to toe off were collected using integrated force plates (Forcelink, 12 channels, sample frequency $1000 \mathrm{~Hz}$ ). Participants were able to self-adjust treadmill speed via a feedback-regulated algorithm in D-flow (Motekforce Link, Amsterdam, the Netherlands). One familiarization session was performed in order to become accustomed to self-paced treadmill walking and the virtual reality hallway environment. The first GRAILbased 6MWT was performed after the familiarization session. Both the familiarization and the first GRAIL-based 6MWT were conducted during pre-rehabilitation assessment. Patients performed the second GRAIL-based 6MWT between pre-rehabilitation assessment and the first week of pulmonary rehabilitation. Healthy elderly performed two GRAIL-based 6MWTs within one day with at least 45 minutes of rest in between the two tests. Perceived dyspnea and fatigue were assessed before and after the GRAIL-based 6MWT using a BORG scale. Heart rate and pulse oxygen saturation $\left(\mathrm{SpO}_{2}\right)$ levels were measured prior and post each GRAlL-based 6MWT using pulse oximetry (Nonin, Care Fusion, San Diego, USA). The instructions of the GRAIL-based 6MWT were provided according to the European Respiratory Society/American Thoracic Society guidelines [21].

The GRAIL-based 6MWT with the longest walk distance for each subject was used for data processing and analysis. The first 60 seconds of the data were excluded to minimize start-up effects and 15 seconds prior to the end of the test were excluded to minimize deceleration of the treadmill speed towards the end of the test (Supplementary Material 3.A). All steps were included for analysis. Gait parameters were determined using a custom program in Matlab (MathWorks Inc., Natick, USA; Supplementary Material 3.B). We computed the following spatiotemporal gait characteristics: walking speed ( $\mathrm{m} / \mathrm{s}$ ), cadence (steps/min), double support time (s), stride time $(\mathrm{s})$, stride length $(\mathrm{m})$ and step width $(\mathrm{m})$. Step time $(\mathrm{s})$, stance time $(\mathrm{s})$, swing time $(\mathrm{s})$ and step length $(\mathrm{m})$ were com- 


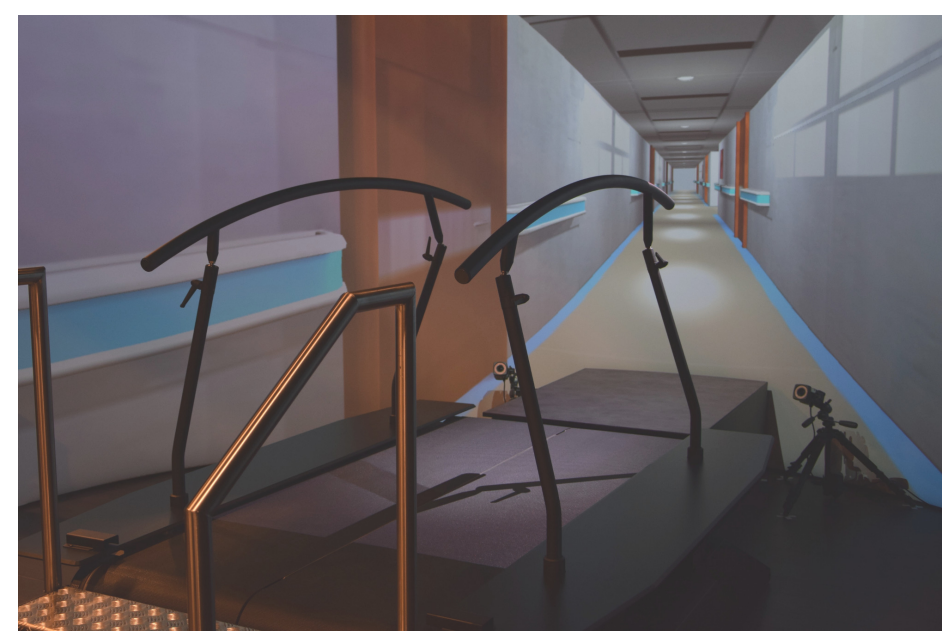

Figure 3.1. The GRAIL system

puted for left and right separately. Double support time was calculated as the duration of both feet on the force plates. Stride time was calculated as the time from one heel contact to the next ipsilateral heel contact. Stride length was defined as the distance between the toe marker and the ipsilateral toe marker at each heel contact in the anterior-posterior direction. Step width was defined as the distance between the toe marker in mediolateral direction between both feet at heel strike. Step time was calculated as the time from one heel contact to the contralateral heel contact. Stance time was calculated as the duration between heel strike and toe off of the ipsilateral leg. Swing time was calculated as the duration between toe of and heel strike of the ipsilateral leg. Step length was defined as the distance between the toe marker in anteroposterior direction between both feet at heel strike (Figure 3.3).

To assess the magnitude of variability in spatiotemporal gait characteristics, coefficient of variation ([standard deviation of the gait characteristic divided by the mean] x 100\%) was calculated for double support time, stride time, stride length, step width, step time, stance time, swing time and step length. In addition, we included commonly reported parameters of the 6MWT, such as 6MWD, mean walking speed, BORG dyspnea score, BORG fatigue score, $\mathrm{SpO}_{2}$ level and heart rate.

\section{Statistical analyses}

Sample size and power sample size calculations were based on outcomes of Annegarn et al. [3] using the inter-stride trunk acceleration variability of patients with COPD and 


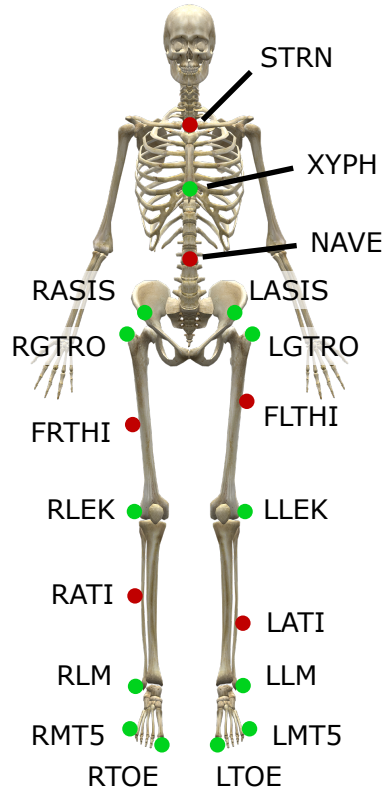

Figure 3.2. The Human Body Model of the lower limb. Anatomical landmarks are the sternum (STRN), xiphoid (XYPH), navel (NAVE), T10, sacral bone, anterior and posterior superior iliac spine (ASIS and PSIS), greater trochanter of the femur (TROCH), lateral between the greater trochanter and lateral femoral epicondyle (LTHI), lateral epicondyle of the knee (LEK), lateral between the lateral femoral epicondyle and lateral malleolus (ATI), lateral malleoli (LM), heel (HEE), tip of the first metatarsal (TOE) and the fifth metatarsal heads (MT5).

healthy elderly. Thirty-five participants in each group would provide $90 \%$ power at alpha 0.05 (two-tailed) to detect differences between patients with COPD and healthy elderly (63.2 $\pm 14.0 \%$ and $73.7 \pm 12.5 \%$, respectively). As spatiotemporal gait characteristics during the GRAIL-based 6MWT have not been assessed, larger sample sizes were used for analyses.

All variables were checked for outliers (Supplementary Material 3.C) and normality distribution using the Shapiro Wilk test. Differences between patients with COPD and healthy elderly were studied using independent samples T-tests and non-parametric independent T-tests. A posteriori, the first sub-analysis of 14 patients and 14 healthy elderly with comparable walking distances was performed to assess the differences between the groups independent of walking speed. To assess possible influences of muscular dysfunction on gait, a second sub-analysis was conducted to assess differences in spatiotemporal gait characteristics between patients with low (isokinetic peak torque $<70 \%$ predicted) and high (isokinetic peak torque $>70 \%$ predicted) quadriceps muscle strength in COPD. This 


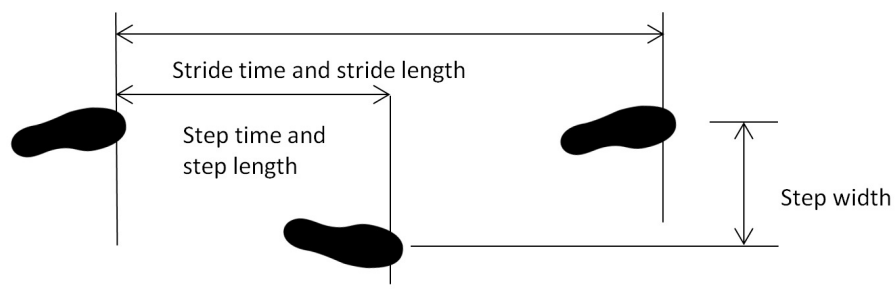

Figure 3.3. Spatiotemporal gait characteristics

value corresponded to the cut-off value of 2 standard deviations below the mean isokinetic peak torque for males and females in age group 60 years [19]. In the third subanalysis, we compared spatiotemporal gait characteristics between patients with mild to moderate COPD (GOLD I-II) and severe to very severe COPD (GOLD III-IV). Mean values, mean differences (MD), standard deviations, coefficients of variation and confidence intervals $(\mathrm{Cl})$ were calculated. A $p$-value below the conventional level of significance $(p<0.01)$ was considered statistically significant. Statistical analyses were performed using SPSS, version 22.0 (IBM, New York, USA).

\section{Results}

\section{Demographics}

A sample of 80 patients and 38 healthy elderly were analyzed for this study. Baseline characteristics of the study population are presented in Table 3.1. Majority of the patients had moderate to severe COPD.

The GRAIL-based 6MWT and spatiotemporal gait characteristics

Patients with COPD achieved on average a shorter 6MWD compared to healthy elderly (494 $\pm 80 \mathrm{~m}$ and $689 \pm 64 \mathrm{~m}, p<0.001$, respectively, Table 3.2). Oxygen saturation level decreased significantly during the GRAIL-based 6MWT in patients $(-2.7 \pm 4.3 \%, p<0.001)$ and exercise induced oxygen desaturation was found in $20 \%$ of the patients $\left(\mathrm{SpO}_{2}\right.$ nadir $\leq 88 \%$ ). Patients with COPD showed less increase in heart rate and experienced more dyspnea and fatigue after the GRAIL-based 6MWT compared to healthy elderly.

Patients with COPD demonstrated decreased cadence compared to healthy elderly (MD: 
Table 3.1. Demographics of subjects

\begin{tabular}{|c|c|c|c|c|}
\hline \multicolumn{2}{|l|}{ Parameter } & $\begin{array}{l}\text { COPD } \\
n=80\end{array}$ & $\begin{array}{c}\text { Healthy } \\
n=38\end{array}$ & $p$-value \\
\hline \multicolumn{2}{|l|}{ Age, years } & $62.3(7.2)$ & $62.1(6.3)$ & 0.718 \\
\hline \multicolumn{2}{|l|}{ Males, $n$} & $48(60.0)$ & $24(63.2)$ & \\
\hline \multicolumn{2}{|l|}{ Weight, kg } & $75.9(16.9)^{\ddagger}$ & $79.3(13.0)$ & 0.273 \\
\hline \multicolumn{2}{|l|}{ Height, $m$} & $1.70(0.09)$ & $1.72(0.08)^{\ddagger}$ & 0.182 \\
\hline \multicolumn{2}{|l|}{$\mathrm{BMI}, \mathrm{kg} / \mathrm{m}^{2}$} & $26.3(5.1)$ & $26.8(3.1)$ & 0.444 \\
\hline \multicolumn{2}{|l|}{$\mathrm{FEV}_{1} / \mathrm{FVC}$} & $0.41(0.11)$ & $0.77(0.05)^{\ddagger}$ & $<0.001$ \\
\hline \multicolumn{2}{|l|}{$\mathrm{FEV}_{1} \%$ predicted } & $55.8(19.4)$ & $118.6(16.5)^{\ddagger}$ & $<0.001$ \\
\hline \multicolumn{2}{|l|}{ Never smoker, $n$} & $1(1.3)$ & $14(36.8)$ & \\
\hline \multicolumn{2}{|l|}{ Former smoker, $n$} & $63(78.8)$ & $23(60.5)$ & \\
\hline \multicolumn{2}{|l|}{ Current smoker, $n$} & $6(7.5)$ & $1(2.6)$ & \\
\hline \multirow[t]{4}{*}{ GOLD group, $n$} & 1 & $10(12.5)$ & & \\
\hline & II & $35(43.8)$ & & \\
\hline & III & $29(36.3)$ & & \\
\hline & IV & $6(7.5)$ & & \\
\hline
\end{tabular}

Note: Data are presented as number (\%) or mean (SD), unless otherwise stated. Abbreviations: COPD, chronic obstructive pulmonary disease; $\mathrm{BMI}$, body mass index; $\mathrm{FEV}_{1}$, forced expiratory volume in $1 \mathrm{~s}$; FVC, forced vital capacity; GOLD, Global Initiative for Chronic Obstructive Lung Disease.

*: Significant difference between patients with COPD and healthy elderly.

$\dagger$ : Non-normal distributed variable.

Table 3.2. Best GRAIL-based 6MWT outcomes in patients with COPD and healthy elderly

\begin{tabular}{|c|c|c|c|}
\hline Parameter & COPD & Healthy & $p$-value \\
\hline 6MWD, m & $493.5(79.7)$ & $689.3(64.3)$ & $<0.001^{*}$ \\
\hline Walking speed, $\mathrm{m} / \mathrm{s}$ & $1.4(0.2)$ & $1.9(0.2)$ & $<0.001^{*}$ \\
\hline Pre $\mathrm{SpO}_{2}, \%$ & $95.1(1.5)^{\dagger}$ & $97.1(1.0)^{\dagger}$ & $<0.001^{*}$ \\
\hline Post $\mathrm{SpO}_{2}, \%$ & $92.3(4.6)^{\# \dagger}$ & $97.1(1.2)^{\dagger}$ & $<0.001^{*}$ \\
\hline Pre HR, bpm & $82.0(13.9)$ & $65.9(11.9)$ & $<0.001^{*}$ \\
\hline Post HR, bpm & $103.0\left(18.1^{\#}\right.$ & $98.6\left(22.7^{\#}\right.$ & 0.295 \\
\hline Pre dyspnea, score & $1.2(1.2)^{\dagger}$ & $0.2(0.3)^{\dagger}$ & $<0.001^{*}$ \\
\hline Post dyspnea, score & $4.8(2.3)^{\# \dagger}$ & $1.1(1.0)^{\# \dagger}$ & $<0.001^{*}$ \\
\hline Pre fatigue, score & $1.3(1.3)^{\dagger}$ & $0.3(0.4)^{\dagger}$ & $<0.001^{*}$ \\
\hline Post fatigue, score & $4.5(2.3)^{\# \dagger}$ & $1.3(1.1)^{\# \dagger}$ & $<0.001^{*}$ \\
\hline
\end{tabular}

Note: Data are presented as mean (SD). Abbreviations: COPD, chronic obstructive pulmonary disease; 6MWT, 6-minute walk test; $6 \mathrm{MWD}$, 6-minute walk distance; $\mathrm{SpO}_{2}$, peripheral capillary oxygen saturation; $\mathrm{HR}$, heart rate; bpm, beats per minute.

*: Significant difference between patients with COPD and healthy elderly.

\#: Significant difference between pre and post values.

t: Non-normal distributed variable. 
-17.36 steps/min, Cl: -21.10- -13.61, $p<0.001$, Table 3.3). Mean duration of temporal gait characteristics was significantly longer in patients with COPD, while stride length was shorter. No differences were found between left and right spatiotemporal gait characteristics. Patients show increased variability in double support time (MD: 1.18\%, Cl: 0.691.66, $p=0.001$ ), stride length (MD: $1.82 \%, \mathrm{Cl}: 1.39-2.25, p<0.001$ ), stance time (left MD: $0.58 \%, \mathrm{Cl}: 0.29-0.87, p=0.002$; right $\mathrm{MD}: 0.57 \%, \mathrm{Cl}: 0.28-0.86, p=0.003)$, and step length (left MD: 1.77\%, Cl: 1.32-2.22, $p<0.001$; right $\mathrm{MD}: 1.74 \%, \mathrm{Cl}: 1.29-2.19, p<0.001$ ). Step width variability did not reach a statistical significant difference between the groups (MD: $-2.98 \%, \mathrm{Cl}$ : $-5.39--0.57, p=0.011)$. The distribution of spatiotemporal gait characteristics for both groups is presented in Supplementary Material 3.D.

\section{Sub-analyses}

Sub-analysis of 14 patients and 14 healthy elderly subjects with comparable walking distances was performed to assess differences between the groups independent of walking speed (MD: -10.2 m, Cl: -36.7-16.3, p=0.437, Table 3.4). Heart rate, dyspnea and fatigue scores significantly differed in pre and post exercise testing within each group and between the groups. Mean spatiotemporal gait characteristics in patients with COPD did not differ with healthy elderly. Variability in gait characteristics showed differences in stride length. (MD: $0.98 \%, \mathrm{Cl}: 0.35-1.61, p=0.003$ ) and partly in step length (left MD: 1.07\%, Cl: 0.36-1.78, $p=0.005$; right MD: $1.10 \%, \mathrm{Cl}: 0.23-1.97, p=0.014$ ) between patients and healthy subjects (Table 3.5 ). No asymmetry was found between left and right spatiotemporal gait characteristics. Distribution of spatiotemporal gait characteristics for both groups is presented in Supplementary Material 3.E.

In the second sub-analysis, the influence of muscular dysfunction on gait characteristics was assessed within the COPD group. Female COPD subjects were less represented in the high quadriceps muscle strength group $(n=8)$ compared to male COPD subjects in the high muscle strength group $(n=25)$. Therefore, comparisons between quadriceps muscle strength were conducted for males and females separately (Table 3.6). Difference in muscle strength in male subjects was $-23.64 \%$ predicted ( $\mathrm{Cl}:-29.7--17.6, p<0.001)$ and $29.7 \%$ predicted ( $\mathrm{Cl}:-35.5--24.0, p<0.001)$ in female subjects. No significant differences in mean spatiotemporal gait characteristics were found between low and high quadriceps muscle strength groups within male and female subjects.

In the third sub-analysis, the influence of the degree of airflow limitation on gait characteristics was assessed within the COPD group. Spatiotemporal gait characteristics were compared between patients with mild-moderate COPD $(68.9 \pm 1.9 \%$ predicted $)$ and severevery severe COPD (38.0 $\pm 8.0 \%$ predicted; Table 3.7$)$. Mean swing time was significantly different between the groups (left MD: -0.02 s, $\mathrm{Cl}$ : $-0.04--0.01, p=0.003$; right MD: -0.02 
Table 3.3. Spatiotemporal gait characteristics in patients with COPD and healthy elderly

\begin{tabular}{|c|c|c|c|c|}
\hline Parameter & & COPD & Healthy & $p$-value \\
\hline \multicolumn{5}{|c|}{ Mean values } \\
\hline & Cadence, steps/min & $118.6(10.3)$ & $136.0(8.0)$ & $<0.001^{*}$ \\
\hline & Double support time, $\mathrm{s}$ & $0.28(0.04)$ & $0.24(0.02)$ & $<0.001^{*}$ \\
\hline & Stride time, $\mathrm{s}$ & $1.02(0.09)$ & $0.89(0.05)$ & $<0.001^{*}$ \\
\hline & Stride length, $\mathrm{m}$ & $1.43(0.18)$ & $1.73(0.14)$ & $<0.001^{*}$ \\
\hline & Step width, m & $0.18(0.04)$ & $0.17(0.05)$ & 0.056 \\
\hline \multirow[t]{4}{*}{ Left } & Step time, s & $0.51(0.04)$ & $0.44(0.03)$ & $<0.001^{*}$ \\
\hline & Stance time, s & $0.65(0.06)$ & $0.56(0.03)$ & $<0.001^{*}$ \\
\hline & Swing time, s & $0.37(0.04)$ & $0.33(0.02)$ & $<0.001^{*}$ \\
\hline & Step length, $\mathrm{m}$ & $0.71(0.09)$ & $0.86(0.07)$ & $<0.001^{*}$ \\
\hline \multirow[t]{4}{*}{ Right } & Step time, s & $0.51(0.05)$ & $0.44(0.03)$ & $<0.001^{*}$ \\
\hline & Stance time, s & $0.65(0.06)$ & $0.56(0.03)$ & $<0.001^{*}$ \\
\hline & Swing time, s & $0.37(0.04)$ & $0.33(0.02)$ & $<0.001^{*}$ \\
\hline & Step length, m & $0.72(0.10)$ & $0.87(0.07)$ & $<0.001^{*}$ \\
\hline & Cadence, \% & $2.99(1.26)^{\dagger}$ & $2.65(0.73)^{\dagger}$ & 0.154 \\
\hline & Double support time, \% & $6.37(1.90)^{\dagger}$ & $5.19(0.77)$ & $0.001^{*}$ \\
\hline & Stride time, \% & $1.97(0.77)^{\dagger}$ & $1.64(0.32)$ & 0.102 \\
\hline & Stride length, \% & $3.87(1.78)^{\dagger}$ & $2.03(0.54)^{\dagger}$ & $<0.001^{*}$ \\
\hline & Step width, \% & $13.97(5.35)^{\dagger}$ & $16.95(7.66)^{\dagger}$ & 0.011 \\
\hline \multirow[t]{4}{*}{ Left } & Step time, \% & $2.54(0.82)^{\dagger}$ & $2.23(0.46)^{\dagger}$ & 0.087 \\
\hline & Stance time, \% & $2.94(1.05)^{\dagger}$ & $2.36(0.54)^{\dagger}$ & $0.002^{*}$ \\
\hline & Swing time, \% & $2.36(0.56)^{\dagger}$ & $2.70(0.46)^{\dagger}$ & 0.580 \\
\hline & Step length, \% & $4.35(1.85)^{\dagger}$ & $2.58(0.58)$ & $<0.001^{*}$ \\
\hline \multirow[t]{4}{*}{ Right } & Step time, \% & $2.58(0.82)^{\dagger}$ & $2.26(0.50)^{\dagger}$ & 0.081 \\
\hline & Stance time, \% & $2.91(1.06)^{\dagger}$ & $2.34(0.53)$ & $0.003^{*}$ \\
\hline & Swing time, \% & $2.41(0.65)^{\dagger}$ & $2.28(0.47)^{\dagger}$ & 0.561 \\
\hline & Step length, \% & $4.35(1.82)^{\dagger}$ & $2.61(0.61)^{\dagger}$ & $<0.001^{*}$ \\
\hline
\end{tabular}

Note: Data are presented as mean (SD). Abbreviation: COPD, chronic obstructive pulmonary disease.

*: Significant difference between patients with COPD and healthy elderly.

†: Non-normal distributed variable. 
Table 3.4. Sub-analysis of the GRAIL-based 6MWT outcomes in groups with comparable walking speeds

\begin{tabular}{llll}
\hline Parameter & $\begin{array}{l}\text { COPD } \\
n=14\end{array}$ & $\begin{array}{c}\text { Healthy } \\
n=14\end{array}$ & $p$-value \\
\hline $\mathrm{FEV}_{1} / \mathrm{FVC}$ & $44.3(10.1)$ & $77.7(5.8)$ & $<0.001$ \\
$\mathrm{FEV}_{1}, \%$ predicted & $64.2(18.2)$ & $116.7(23.1)$ & $<0.001$ \\
6MWD, m & $611.8(30.2)$ & $622.0(37.7)$ & 0.437 \\
Walking speed, m/s & $1.7(0.1)$ & $1.7(0.1)$ & 0.710 \\
Pre $\mathrm{SpO}_{2}, \%$ & $95.8(1.7)$ & $97.4(0.6)^{\dagger}$ & $0.003^{*}$ \\
Post $\mathrm{SpO}_{2}, \%$ & $94.2(3.7)^{\dagger}$ & $97.0(1.0)^{\dagger}$ & 0.015 \\
Pre $\mathrm{HR}, \mathrm{bpm}$ & $78.7(14.8)$ & $65.6(9.9)$ & 0.010 \\
Post $\mathrm{HR}$, bpm & $108.8(19.1)^{\#}$ & $92.4(18.3)^{\#}$ & 0.028 \\
Pre dyspnea, score & $1.1(0.9)^{\dagger}$ & $0.2(0.3)^{\dagger}$ & $0.001^{*}$ \\
Post dyspnea, score & $5.0(2.2)^{\# \dagger}$ & $0.8(0.5)^{\# \dagger}$ & $<0.001^{*}$ \\
Pre fatigue, score & $0.9(0.9)^{\dagger}$ & $0.3(0.5)^{\dagger}$ & $0.006^{*}$ \\
Post fatigue, score & $4.8(2.4)^{\# \dagger}$ & $0.9(0.9)^{\# \dagger}$ & $<0.001^{*}$ \\
\hline
\end{tabular}

Note: Data are presented as mean (SD). Abbreviations: COPD, chronic obstructive pulmonary disease; FEV 1 , forced expiratory volume in $1 \mathrm{~s}$; FVC, forced vital capacity; 6MWT, 6-minute walk test; 6MWD, 6-minute walk distance; $\mathrm{SpO}_{2}$, peripheral capillary oxygen saturation; $\mathrm{HR}$, heart rate; bpm, beats per minute.

*: Significant difference between patients with COPD and healthy elderly.

\#: Significant difference between pre and post values.

$\dagger$ : Non-normal distributed variable.

s, Cl:-0.04 -0.01, $p=0.003)$.

\section{Discussion}

This study demonstrates that patients with COPD have different spatiotemporal gait characteristics as compared to healthy elderly during a self-paced treadmill based 6MWT. Patients achieved shorter walk distances with a lower cadence and increased duration of temporal gait characteristics. Patients took shorter steps and showed increased variability in double support time, stride length, stance time and step length. Sub-analysis showed that differences in mean spatiotemporal gait characteristics are mainly attributed to differences in walking speed between the groups. However, variability in stride length remained higher in patients with COPD compared to healthy elderly in the sub-analysis of subject groups with comparable walking speeds. Stratifying patients by quadriceps muscle strength did not distinguish differences in spatiotemporal gait characteristics. Patients with mild-moderate COPD showed decreased swing time as compared to severe-very severe COPD.

This is the first study to investigate spatiotemporal gait characteristics during a self-paced 
Table 3.5. Sub-analysis of the spatiotemporal gait characteristics in groups with comparable walking speeds

\begin{tabular}{|c|c|c|c|c|}
\hline Parameter & & $\begin{array}{c}\text { COPD } \\
n=14\end{array}$ & $\begin{array}{c}\text { Healthy } \\
n=14\end{array}$ & $p$-value \\
\hline \multicolumn{5}{|c|}{ Mean values } \\
\hline & Cadence, steps/min & $128.40(12.00)$ & $132.00(4.86)$ & 0.306 \\
\hline & Double support time, s & $0.25(0.02)^{\dagger}$ & $0.24(0.02)$ & 0.227 \\
\hline & Stride time, $\mathrm{s}$ & $0.94(0.08)$ & $0.91(0.03)$ & 0.212 \\
\hline & Stride length, $\mathrm{m}$ & $1.62(0.17)$ & $1.59(0.07)$ & 0.552 \\
\hline & Step width, m & $0.18(0.04)$ & $0.16(0.05)^{\dagger}$ & 0.260 \\
\hline \multirow[t]{4}{*}{ Left } & Step time, s & $0.47(0.04)$ & $0.46(0.02)$ & 0.209 \\
\hline & Stance time, s & $0.59(0.04)$ & $0.57(0.03)$ & 0.158 \\
\hline & Swing time, s & $0.35(0.04)$ & $0.34(0.02)$ & 0.350 \\
\hline & Step length, m & $0.82(0.09)$ & $0.80(0.04)$ & 0.512 \\
\hline \multirow[t]{4}{*}{ Right } & Step time, s & $0.47(0.04)$ & $0.45(0.02)$ & 0.213 \\
\hline & Stance time, $\mathrm{s}$ & $0.60(0.05)$ & $0.58(0.03)$ & 0.180 \\
\hline & Swing time, s & $0.34(0.04)$ & $0.33(0.02)$ & 0.281 \\
\hline & Step length, $\mathrm{m}$ & $0.82(0.08)$ & $0.80(0.04)$ & 0.422 \\
\hline \multicolumn{5}{|l|}{$\begin{array}{l}\text { Coefficient } \\
\text { of variation }\end{array}$} \\
\hline & Cadence, $\%$ & $2.81(0.84)$ & $2.44(0.87)^{\dagger}$ & 0.104 \\
\hline & Double support time, \% & $5.81(1.36)$ & $4.74(0.75)$ & 0.015 \\
\hline & Stride time, $\%$ & $1.74(0.48)$ & $1.45(0.27)$ & 0.067 \\
\hline & Stride length, $\%$ & $3.03(0.98)$ & $2.04(0.59)$ & $0.003^{*}$ \\
\hline & Step width, \% & $16.17(5.92)^{\dagger}$ & $15.09(4.82)$ & 0.963 \\
\hline \multirow[t]{4}{*}{ Left } & Step time, \% & $2.41(0.81)^{\dagger}$ & $1.93(0.27)$ & 0.178 \\
\hline & Stance time, \% & $2.64(0.90)^{\dagger}$ & $2.09(0.34)$ & 0.050 \\
\hline & Swing time, \% & $2.35(0.70)^{\dagger}$ & $2.02(0.28)$ & 0.352 \\
\hline & Step length, \% & $3.61(1.12)$ & $2.54(0.63)$ & $0.005^{*}$ \\
\hline \multirow[t]{4}{*}{ Right } & Step time, \% & $2.43(0.85)^{\dagger}$ & $2.05(0.40)$ & 0.265 \\
\hline & Stance time, \% & $2.62(0.89)^{\dagger}$ & $2.02(0.33)$ & 0.062 \\
\hline & Swing time, $\%$ & $2.35(0.73)^{\dagger}$ & $2.10(0.33)$ & 0.839 \\
\hline & Step length, \% & $3.72(1.40)^{\dagger}$ & $2.62(0.67)$ & 0.014 \\
\hline
\end{tabular}

Note: Data are presented as mean (SD). Abbreviation: COPD, chronic obstructive pulmonary disease.

*: Significant difference between patients with COPD and healthy elderly.

†: Non-normal distributed variable. 


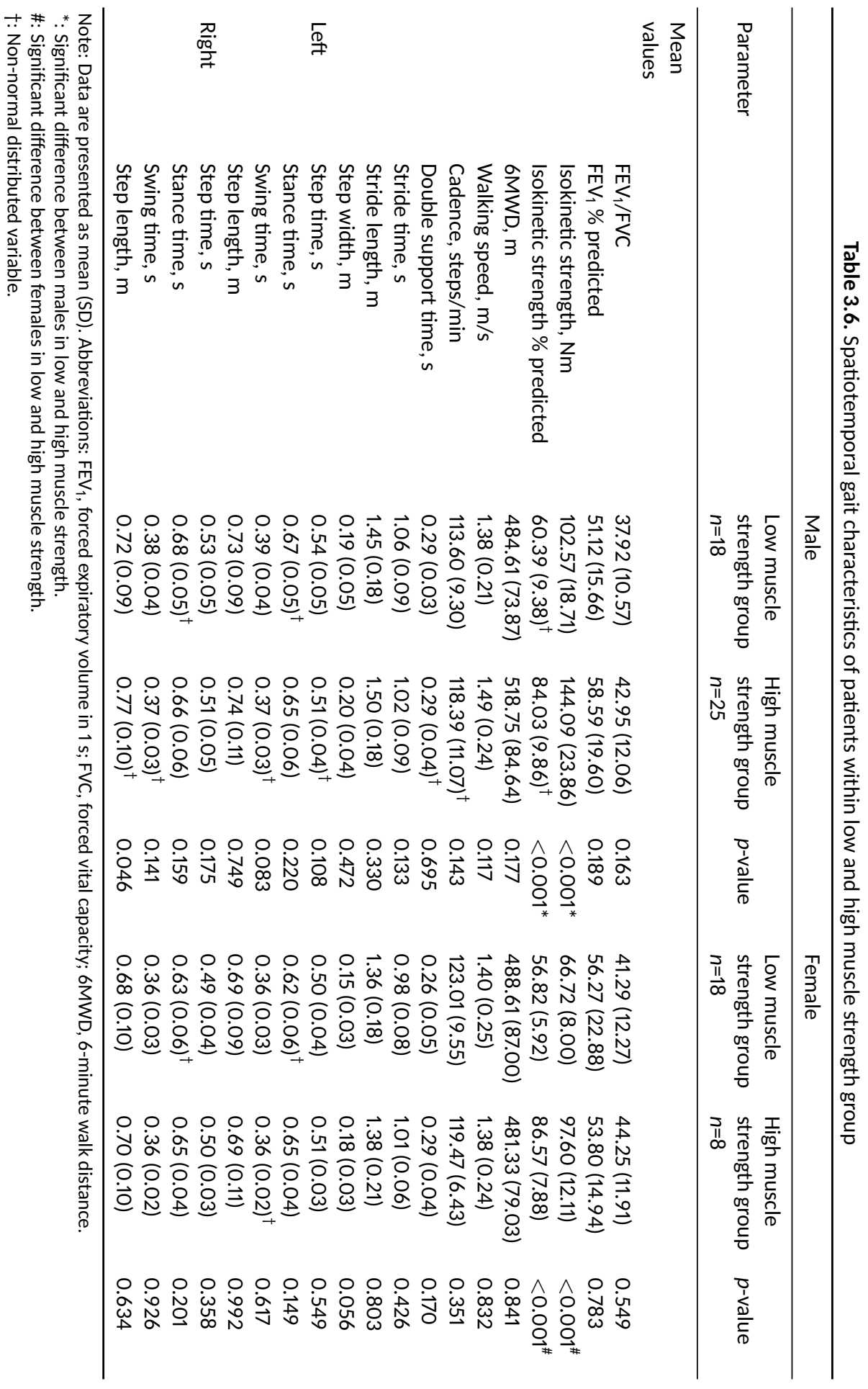


Table 3.7. Spatiotemporal gait characteristics of patients within mild-moderate and severe-very severe COPD

\begin{tabular}{|c|c|c|c|c|}
\hline Parameter & & $\begin{array}{l}\text { Mild-moderate } \\
\text { COPD } \\
n=45\end{array}$ & $\begin{array}{l}\text { Severe-very sev } \\
\text { COPD } \\
n=35\end{array}$ & $p$-value \\
\hline \multicolumn{5}{|c|}{ Mean values } \\
\hline & $\mathrm{FEV}_{1} / \mathrm{FVC}$ & $47.98(8.55)$ & $32.98(8.86)^{\dagger}$ & $<0.001^{*}$ \\
\hline & $\mathrm{FEV}_{1} \%$ predicted & $69.70(13.24)$ & $37.98(8.04)$ & $<0.001^{*}$ \\
\hline & 6MWD, m & 507.05 (87.98) & $480.55(65.70)$ & 0.141 \\
\hline & Walking speed, $\mathrm{m} / \mathrm{s}$ & $1.45(0.25)$ & $1.37(0.18)$ & 0.093 \\
\hline & Cadence, steps/min & $120.54(10.84)$ & $116.12(9.00)$ & 0.055 \\
\hline & Double support time, s & $0.29(0.03)$ & $0.28(0.04)$ & 0.234 \\
\hline & Stride time, $\mathrm{s}$ & $1.00(0.09)$ & $1.04(0.08)$ & 0.048 \\
\hline & Stride length, $\mathrm{m}$ & $1.44(0.21)$ & $1.41(0.14)$ & 0.534 \\
\hline & Step width, $\mathrm{m}$ & $0.19(0.04)$ & $0.17(0.04)^{\dagger}$ & 0.043 \\
\hline \multirow[t]{4}{*}{ Left } & Step time, s & $0.51(0.05)$ & $0.52(0.04)$ & 0.088 \\
\hline & Stance time, s & $0.64(0.06)$ & $0.66(0.06)$ & 0.315 \\
\hline & Swing time, s & $0.36(0.04)^{\dagger}$ & $0.38(0.03)$ & $0.002^{*}$ \\
\hline & Step length, $m$ & $0.72(0.10)$ & $0.70(0.08)$ & 0.332 \\
\hline \multirow[t]{4}{*}{ Right } & Step time, s & $0.50(0.05)$ & $0.52(0.04)$ & 0.055 \\
\hline & Stance time, s & $0.65(0.06)$ & $0.66(0.06)$ & 0.313 \\
\hline & Swing time, s & $0.36(0.04)$ & $0.38(0.03)$ & $0.003^{*}$ \\
\hline & Step length, $\mathrm{m}$ & $0.72(0.11)$ & $0.71(0.08)^{\dagger}$ & 0.186 \\
\hline
\end{tabular}

Note: Data are presented as mean (SD). Abbreviations: COPD, chronic obstructive pulmonary disease; FEV forced expiratory volume in $1 \mathrm{~s}$; FVC, forced vital capacity; 6MWD, 6-minute walk distance.

*: Significant difference between mild-moderate COPD and severe-very severe COPD.

†: Non-normal distributed variable. 
treadmill based 6MWT in patients with COPD using the GRAIL. Walking speed and cadence were decreased in patients with COPD, which is in line with previous studies [3, $22,23]$. Reduced gait speed and cadence could reflect respiratory limitations associated with muscular dysfunction in COPD [22, 24, 25]. These gait alterations might be an adaptation mechanism to cope with impaired walking endurance due to the lack of oxygen supply and lower limb muscle weakness. Taking slower steps decreases the oxygen demands of the leg muscles and thus allows for increased walking distance in patients with impaired lung function [26]. Increased step width could be an adaptation to increase the base of support during the double support phase of gait [10]. However, our results did not support differences in step width between patients with COPD and healthy elderly.

Increased variability in double support time, stride length, stance time and step length were found in patients with COPD as compared to healthy elderly. These findings show that patients are less consistent in their walking pattern during the GRAIL-based 6MWT. Step length and stance time variability are increased in older adult fallers as compared to older adult non-fallers [27, 28]. Increased gait variability in COPD could therefore be associated with an increased fall risk within this population. Decreased step width variability in patients with COPD as compared to healthy elderly subjects did not reach a statistical significant difference. However, Yentes et al. [12] suggested that reduced variability in step width may be related to an increased likelihood to a crossover gait. This might indicate that patients are less able to maintain stability, thus predisposing an individual to a fall $[29,30]$.

Variability in spatiotemporal gait characteristics was calculated as the coefficient of variation, describing the magnitude of variation in each spatiotemporal gait characteristic. When examining the individual variations per gait characteristic, differences between the two groups are observed. However, these differences are cancelled out when comparing the mean values in a subgroup with similar walking speeds. Therefore, other measures that do take the temporal structure of variation into account could provide additional insight into the control of the system while walking [31-34]. Future research might investigate temporal fluctuations of gait characteristics in patients with COPD, to determine subtle alterations in gait in patients with COPD and its associations with fall risk.

The first sub-analysis showed increased variability in stride length in patients with COPD compared to healthy elderly, which indicates that patients with COPD are more variable in their stride lengths irrespective of walking speed differences. Patients with COPD seem to display a larger distribution of variability in their gait characteristics as compared to healthy elderly when walking at similar walking speeds. These patients experienced greater elevated heart rates and report higher dyspnea and fatigue scores for both pre and post values compared to healthy elderly. These findings direct to the systemic conse- 
quences of COPD, resulting in muscular dysfunction and deconditioning [5, 35], in which pulmonary rehabilitation has proven to enhance exercise performance in patients with COPD [36]. Patients with COPD are more susceptible to fatigue [37], possibly explaining the increased fatigue scores in our patients as compared to healthy elderly. Gait alterations in patients with COPD could result in decreased exercise capacity and increased symptoms after the 6MWT. Therefore, gait training could be incorporated within pulmonary rehabilitation programs to improve walking ability and symptoms after exercise testing in COPD.

Previous literature reported correlations between the severity of the disease and functional exercise capacity [38, 39]. However, the current study does not provide evidence for influences of COPD severity on spatiotemporal gait characteristics. Airflow limitation might not have translated to alterations in mean spatiotemporal gait characteristics in COPD. This could be explained by that patients were mostly diagnosed as GOLD II (moderate) and III (severe), whereas patients with GOLD I (mild) and IV (very severe) were less represented in the disease severity groups. Skeletal muscle dysfunction, in particular the quadriceps, is well recognized in COPD and is associated with impaired functional capacity, such as the 6MWT [40,41]. Our results however did not support differences in spatiotemporal gait characteristics between patients with low and high quadriceps muscle strength. A possible explanation could be that reduced quadriceps muscle strength is not directly related to impaired spatiotemporal gait characteristics in COPD. Alternatively, more distal located lower limb muscles might be more appropriate to be associated with gait characteristics. Previous studies have demonstrated the importance of the triceps surae for propulsion during gait [42-45]. Weakness in ankle dorsiflexors and plantarflexors has been reported to be greater than weakness present in the quadriceps of patients with COPD [46, 47]. In addition, patients with COPD demonstrate a lack of increase in peak ankle dorsiflexion moment after a fatiguing treadmill protocol [10]. Patients with COPD might therefore experience problems in lowering their forefoot after heel strike. Future studies may examine the association between decreased muscle strength in other muscle groups in the lower limbs and altered gait characteristics.

The current study has some limitations. First, subjects walked on a motorized treadmill, which may impede natural walking pattern and its variability. However, previous studies have reported equivocal findings [48-50]. Self-paced treadmill walking was used to provide a more natural control of the walking speed [14, 51], while virtual reality environment could have created a more realistic environment due to the use of a virtual environment enabling optic flow [15]. In addition, self-paced treadmill walking has been validated for healthy subjects during comfortable walking speed [14, 51]. Second, the sample size of female patients in the high quadriceps muscle strength group is small. Consequently, these 
subjects might not be a comprehensive representation of female patients within the high muscle strength group.

In conclusion, patients with COPD have different spatiotemporal gait characteristics during a self-paced treadmill 6MWT compared to healthy elderly as assessed by the GRAIL. Independent of walking speed, patients with COPD show increased stride length variability during the GRAIL-based 6MWT. Reduced quadriceps strength does not translate into altered spatiotemporal gait characteristics, while COPD severity has impact on swing time. Further research should investigate gait characteristics in patients with COPD using other variability measures, to assess subtle alterations in gait and to enable development of rehabilitation strategies to improve gait, and possibly balance and fall risk in these patients. In addition, other muscle groups in the lower limbs should be taken into account when investigating gait alterations in COPD.

\section{Acknowledgements}

The authors thank the study participants and the staff of CIRO. 


\section{Bibliography}

1. Annegarn, J. et al. Problematic activities of daily life are weakly associated with clinical characteristics in COPD. J Am Med Dir Assoc 13, 284-90 (2012).

2. Pitta, F. et al. Characteristics of physical activities in daily life in chronic obstructive pulmonary disease. Am J Respir Crit Care Med 171, 972-7 (2005).

3. Annegarn, J. et al. Differences in walking pattern during 6-min walk test between patients with COPD and healthy subjects. PLoS One 7, e37329 (2012).

4. Spruit, M. A. et al. Predicting outcomes from 6-minute walk distance in chronic obstructive pulmonary disease. J Am Med Dir Assoc 13, 291-7 (2012).

5. Gosker, H. R. et al. Skeletal muscle fibre-type shifting and metabolic profile in patients with chronic obstructive pulmonary disease. Eur Respir J 19, 617-25 (2002).

6. Wouters, E. F. Chronic obstructive pulmonary disease. 5: systemic effects of COPD. Thorax 57, 1067-70 (2002).

7. Lahousse, L. et al. Gait patterns in COPD: the Rotterdam Study. Eur Respir J 46, 88-95 (2015).

8. Nantsupawat, N. et al. Gait Characteristics in Patients With Chronic Obstructive Pulmonary Disease. J Prim Care Community Health 6, 222-6 (2015).

9. Yentes, J. M. et al. Patients with COPD Walk with Altered Step Time and Step Width Variability as Compared to Healthy Controls. Ann Am Thorac Soc (2017).

10. Yentes, J. M. et al. Gait mechanics in patients with chronic obstructive pulmonary disease. Respir Res 16, 31 (2015).

11. Yentes, J. M. et al. Walking abnormalities are associated with COPD: An investigation of the NHANES III dataset. Respir Med 105, 80-7 (2011).

12. Yentes, J. M. et al. Patients with Chronic Obstructive Pulmonary Disease Walk with Altered Step Time and Step Width Variability as Compared with Healthy Control Subjects. Ann Am Thorac Soc 14, 858-866 (2017).

13. Sloot, L. H., van der Krogt, M. M. \& Harlaar, J. Self-paced versus fixed speed treadmill walking. Gait Posture 39, 478-84 (2014).

14. Plotnik, M. et al. Self-selected gait speed-over ground versus self-paced treadmill walking, a solution for a paradox. J Neuroeng Rehabil 12, 20 (2015).

15. Sloot, L. H., van der Krogt, M. M. \& Harlaar, J. Effects of adding a virtual reality environment to different modes of treadmill walking. Gait Posture 39, 939-45 (2014).

16. Liu, W. Y. et al. Reproducibility and Validity of the 6-Minute Walk Test Using the Gait Real-Time Analysis Interactive Lab in Patients with COPD and Healthy Elderly. PLoS One 11, e0162444 (2016).

17. Helbostad, J. L. \& Moe-Nilssen, R. The effect of gait speed on lateral balance control during walking in healthy elderly. Gait Posture 18, 27-36 (2003).

18. Rutten, E. P. et al. Various Mechanistic Pathways Representing the Aging Process Are Altered in COPD. Chest 149, 53-61 (2016).

19. Borges, O. Isometric and isokinetic knee extension and flexion torque in men and women aged 20-70. Scand J Rehabil Med 21, 45-53 (1989).

20. Van den Bogert, A. J. et al. A real-time system for biomechanical analysis of human movement and muscle function. Med Biol Eng Comput 51, 1069-77 (2013).

21. Holland, A. E. et al. An official European Respiratory Society/American Thoracic Society technical standard: field walking tests in chronic respiratory disease. Eur Respir J 44, 1428-46 (2014).

22. Butcher, S. J., Meshke, J. M. \& Sheppard, M. S. Reductions in functional balance, coordination, and mobility measures among patients with stable chronic obstructive pulmonary disease. J Cardiopulm Rehabil 24, 274-80 (2004).

23. Menard-Rothe, K. et al. Self-selected walking velocity for functional ambulation in patients with endstage emphysema. J Cardiopulm Rehabil 17, 85-91 (1997). 
24. Mador, M. J., Krauza, M. \& Shaffer, M. Effect of exercise training in patients with chronic obstructive pulmonary disease compared with healthy elderly subjects. J Cardiopulm Rehabil Prev 32, 155-62 (2012).

25. Roig, M. et al. Falls in people with chronic obstructive pulmonary disease: an observational cohort study. Respir Med 105, 461-9 (2011).

26. Roig, M. et al. Falls in patients with chronic obstructive pulmonary disease: a call for further research. Respir Med 103, 1257-69 (2009).

27. Hausdorff, J. M. et al. Increased gait unsteadiness in community-dwelling elderly fallers. Arch Phys Med Rehabil 78, 278-83 (1997).

28. Mbourou, G. A., Lajoie, Y. \& Teasdale, N. Step length variability at gait initiation in elderly fallers and non-fallers, and young adults. Gerontology 49, 21-6 (2003).

29. Gabell, A. \& Nayak, U. S. The effect of age on variability in gait. J Gerontol 39, 662-6 (1984).

30. Maki, B. E. Gait changes in older adults: predictors of falls or indicators of fear. J Am Geriatr Soc 45, 313-20 (1997).

31. Hausdorff, J. M. Gait dynamics, fractals and falls: finding meaning in the stride-to-stride fluctuations of human walking. Hum Mov Sci 26, 555-89 (2007).

32. Hausdorff, J. M. et al. Altered fractal dynamics of gait: reduced stride-interval correlations with aging and Huntington's disease. J Appl Physiol (1985) 82, 262-9 (1997).

33. Stergiou, N., Harbourne, R. \& Cavanaugh, J. Optimal movement variability: a new theoretical perspective for neurologic physical therapy. J Neurol Phys Ther 30, 120-9 (2006).

34. Toebes, M. J. et al. Local dynamic stability and variability of gait are associated with fall history in elderly subjects. Gait Posture 36, 527-31 (2012).

35. Gea, J. et al. Muscle dysfunction in chronic obstructive pulmonary disease: update on causes and biological findings. J Thorac Dis 7, E418-38 (2015).

36. Barreiro, E. et al. Guidelines for the evaluation and treatment of muscle dysfunction in patients with chronic obstructive pulmonary disease. Arch Bronconeumol 51, 384-95 (2015).

37. Donaldson, A. V. et al. Muscle function in COPD: a complex interplay. Int J Chron Obstruct Pulmon Dis 7 , 523-35 (2012).

38. Agrawal, M. B. \& Awad, N. T. Correlation between Six Minute Walk Test and Spirometry in Chronic Pulmonary Disease. J Clin Diagn Res 9, OC01-4 (2015).

39. Wijkstra, P. J. et al. Relation of lung function, maximal inspiratory pressure, dyspnoea, and quality of life with exercise capacity in patients with chronic obstructive pulmonary disease. Thorax 49, 468-72 (1994).

40. Gosker, H. R. et al. Skeletal muscle dysfunction in chronic obstructive pulmonary disease and chronic heart failure: underlying mechanisms and therapy perspectives. Am J Clin Nutr 71, 1033-47 (2000).

41. Gosselink, R., Troosters, T. \& Decramer, M. Peripheral muscle weakness contributes to exercise limitation in COPD. Am J Respir Crit Care Med 153, 976-80 (1996).

42. Liu, M. Q. et al. Muscle contributions to support and progression over a range of walking speeds. $J$ Biomech 41, 3243-52 (2008).

43. Murray, M. P. et al. Function of the triceps surae during gait. Compensatory mechanisms for unilateral loss. J Bone Joint Surg Am 60, 473-6 (1978).

44. Requiao, L. F. et al. Quantification of level of effort at the plantarflexors and hip extensors and flexor muscles in healthy subjects walking at different cadences. J Electromyogr Kinesiol 15, 393-405 (2005).

45. Sutherland, D. H., Cooper, L. \& Daniel, D. The role of the ankle plantar flexors in normal walking. J Bone Joint Surg Am 62, 354-63 (1980).

46. Gagnon, P. et al. Distal leg muscle function in patients with COPD. COPD 10, 235-42 (2013).

47. Maddocks, M. et al. Ankle dorsiflexor muscle size, composition and force with ageing and chronic obstructive pulmonary disease. Exp Physiol 99, 1078-88 (2014). 
48. Alton, F. et al. A kinematic comparison of overground and treadmill walking. Clin Biomech (Bristol, Avon) 13, 434-440 (1998).

49. Lee, S. J. \& Hidler, J. Biomechanics of overground vs. treadmill walking in healthy individuals. J Appl Physiol (1985) 104, 747-55 (2008).

50. Riley, P. O. et al. A kinematic and kinetic comparison of overground and treadmill walking in healthy subjects. Gait \& Posture 26, 17-24 (2007).

51. Sloot, L. H., van der Krogt, M. M. \& Harlaar, J. Self-paced versus fixed speed treadmill walking. Gait \& Posture 39 (2013). 



\section{Supplementary Material}

\section{Data processing}

Walking speed of fifteen patients with COPD and fifteen healthy elderly were analysed to assess the duration of acceleration and deceleration of walking speed of subjects during the GRAIL-based 6MWT. Subjects needed time to get accustomed to split-belt, self-paced treadmill walking. Patients with COPD seemed to need a longer period to achieve their preferred walking speed to perform the 6MWT compared to healthy elderly (Figure 3.A.1 and Figure 3.A.2). Therefore, the first minute was removed for analysis. According to the ERS/ATS statement, subjects will be informed that the 6MWT is about to end [1]. To avoid deceleration influencing gait characteristics 15 seconds prior to the end of the 6MWT will be removed for analysis. Therefore, data were cropped from 60 seconds to 345 seconds, to minimize start-up and deceleration effects.

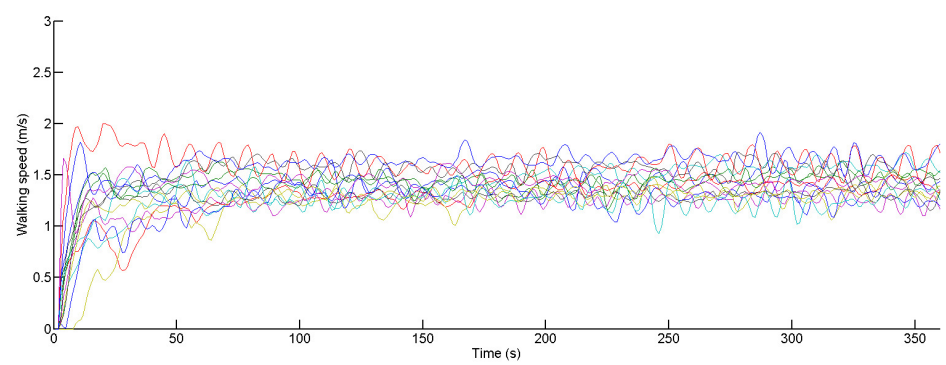

Figure 3.A.1. Walking speed of patients with COPD during the GRAIL-based 6MWT 


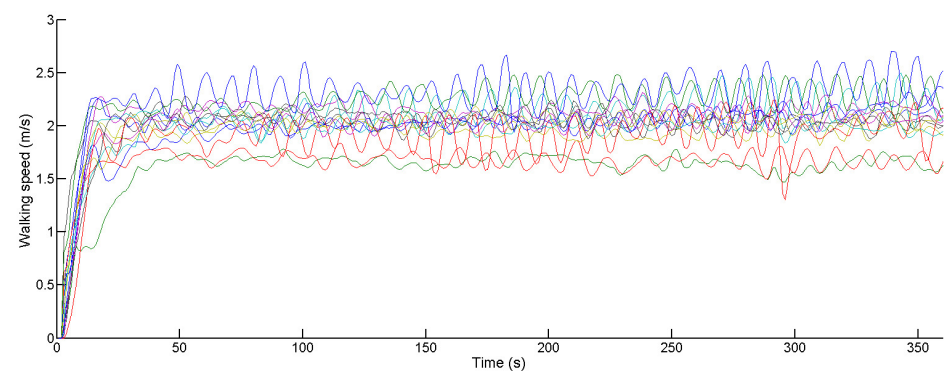

Figure 3.A.2. Walking speed of healthy elderly subjects during the GRAIL-based 6MWT

\section{Data correction and event detection}

All gaps were reconstructed using a spline interpolation in MATLAB (MathWorks Inc., Natick, USA). The tracks were then filtered using a low pass second order Butterworth filter, with a cut-off frequency at $12 \mathrm{~Hz}$. All gaps, exceeding 3 samples at the start and end, were reconstructed using the filtered tracks.

Gait events were calculated using the treadmill velocity based method of Zeni et al. [2]. Foot marker position data were filtered using a second order Butterworth filter, with a cut-off frequency of $10 \mathrm{~Hz}$, before velocity was calculated. Gait events were determined based on force plate data [3-5], using a 50 Newton threshold. The phase shift between the results of both methods was determined for steps in which the force exceeded the threshold. Outliers were detected and removed using a number of samples threshold and when the phase shift exceeded a value of more than 2 times the standard deviation. All events, calculated with the velocity based method, were then corrected with the calculated phase shift. Then spatiotemporal gait characteristics were determined using a custom Matlab program.

\section{Heterogeneity in COPD and healthy elderly group}

The patient group seems to be more heterogeneous in the variability of temporal gait characteristics, stride length and step length compared to healthy elderly (Table 3.3), due 
to the increased standard deviation of these gait characteristics in the patient group for both analyses. Extreme values in mean gait characteristics were more prone in the patient group ( $>1.5$ interquartile range: 13 patients and 3 healthy elderly; $>3.0$ interquartile range: 1 patient). Seventeen patients with COPD could be regarded as extreme values (>1.5 interquartile range) in contrast to 8 healthy elderly subjects. Four patients had interquartile ranges above 3.0 compared to 2 healthy elderly. All subjects were included for analysis, as these values represent the heterogeneity of the subjects in each group. 


\section{Boxplots of spatiotemporal gait characteristics in the total sample}
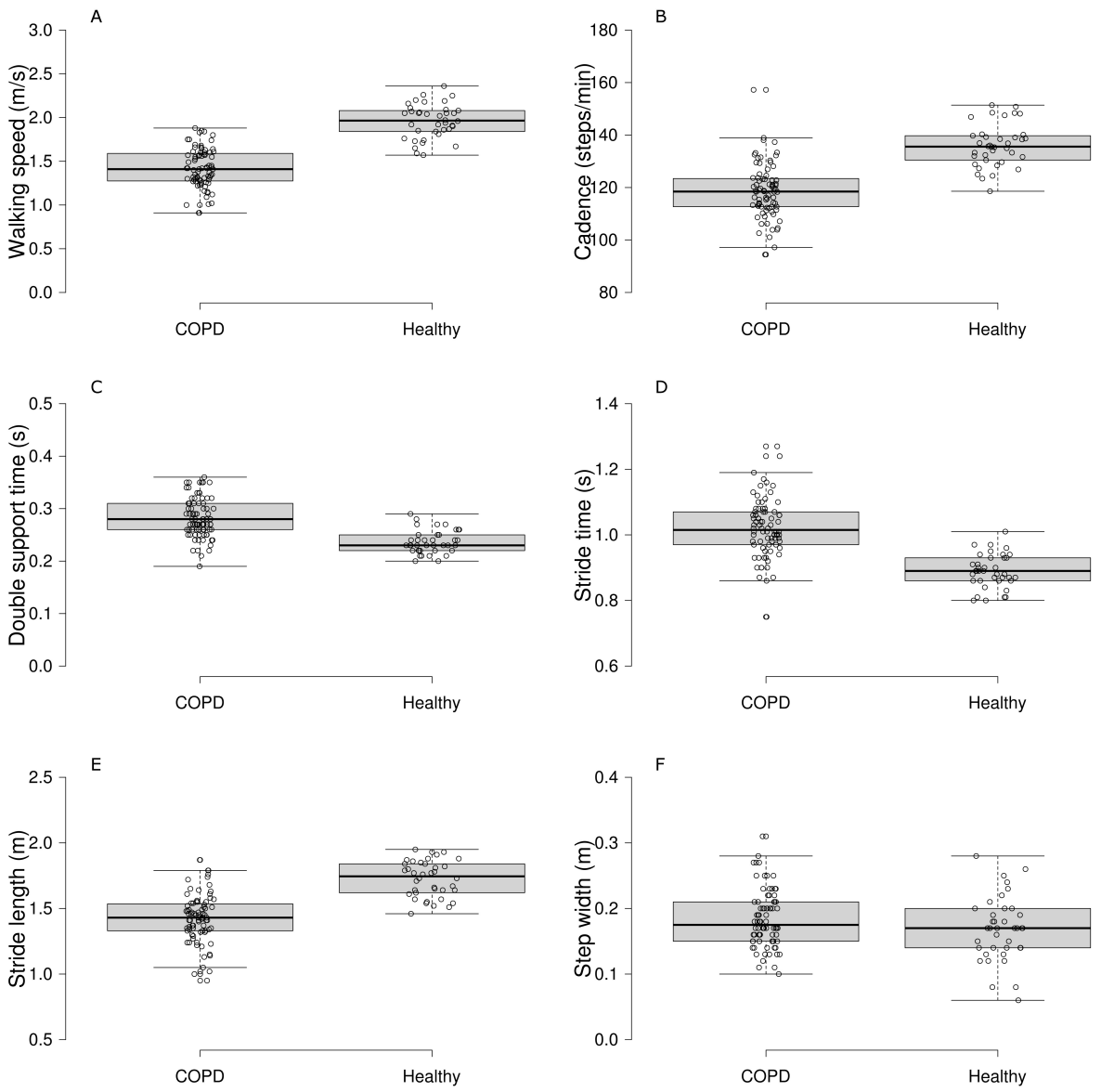

Figure 3.D.1. Boxplots of spatiotemporal gait characteristics in the total sample. Patients with COPD $(n=80)$ and healthy elderly $(n=38)$ are depicted. Centre lines show the medians; box limits indicate the 25th and 75th percentiles; whiskers extend 1.5 times the interquartile range from the 25th and 75th percentiles, outliers are represented by dots. 
A

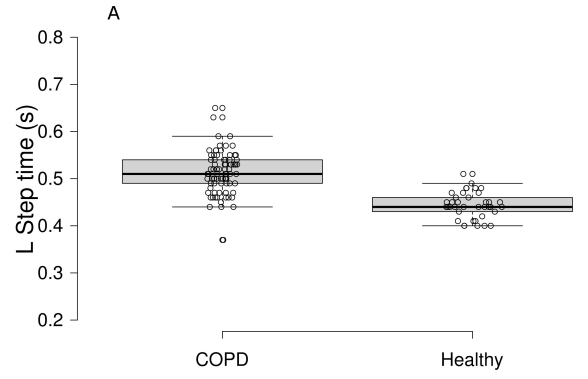

C

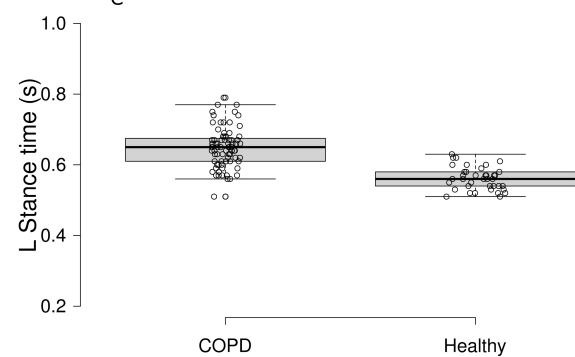

E

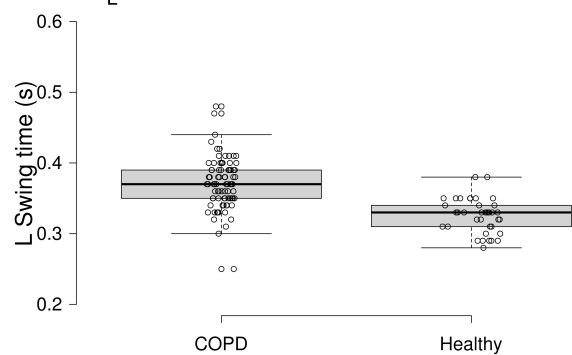

G

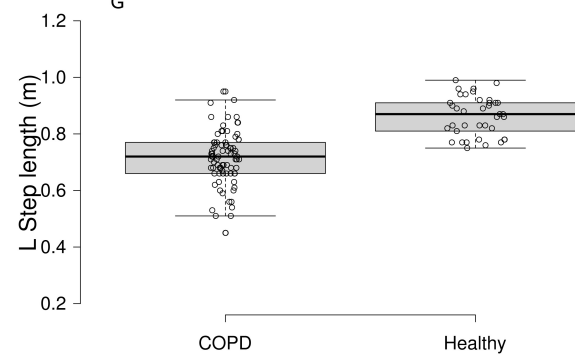

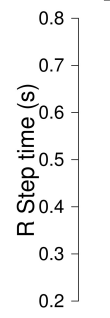

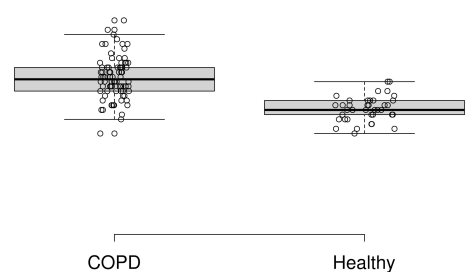

$\mathrm{D}$
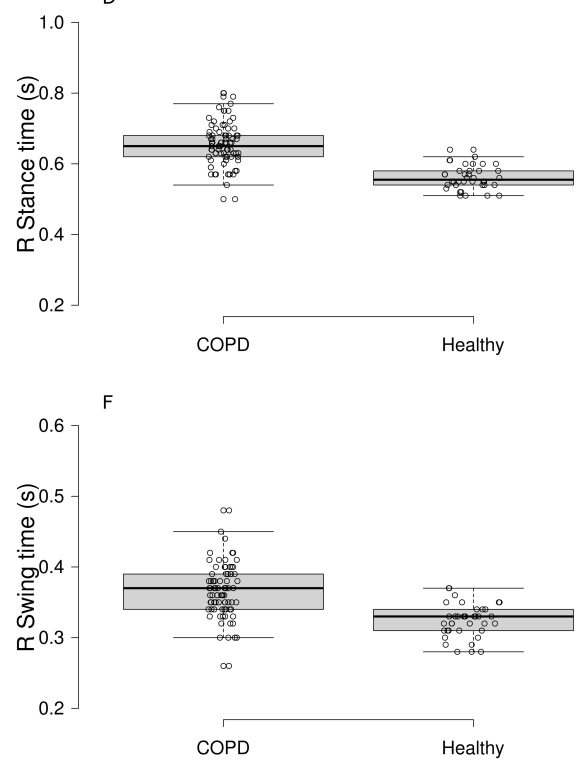

$\mathrm{H}$

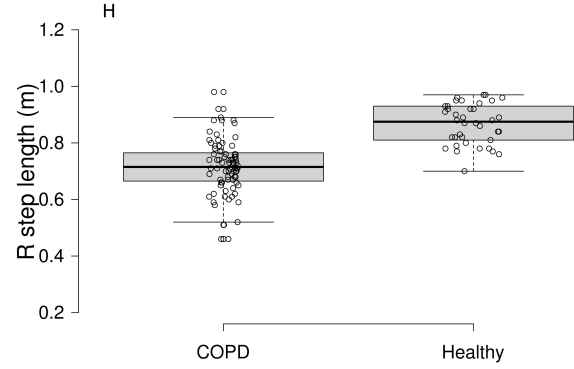

Figure 3.D.2. Boxplots of spatiotemporal gait characteristics separated for left and right side in the total sample. Patients with COPD $(n=80)$ and healthy elderly $(n=38)$ are depicted. Centre lines show the medians; box limits indicate the 25 th and 75th percentiles; whiskers extend 1.5 times the interquartile range from the 25 th and 75 th percentiles, outliers are represented by dots. 


\section{Boxplots of spatiotemporal gait characteristics in the sub-analysis}
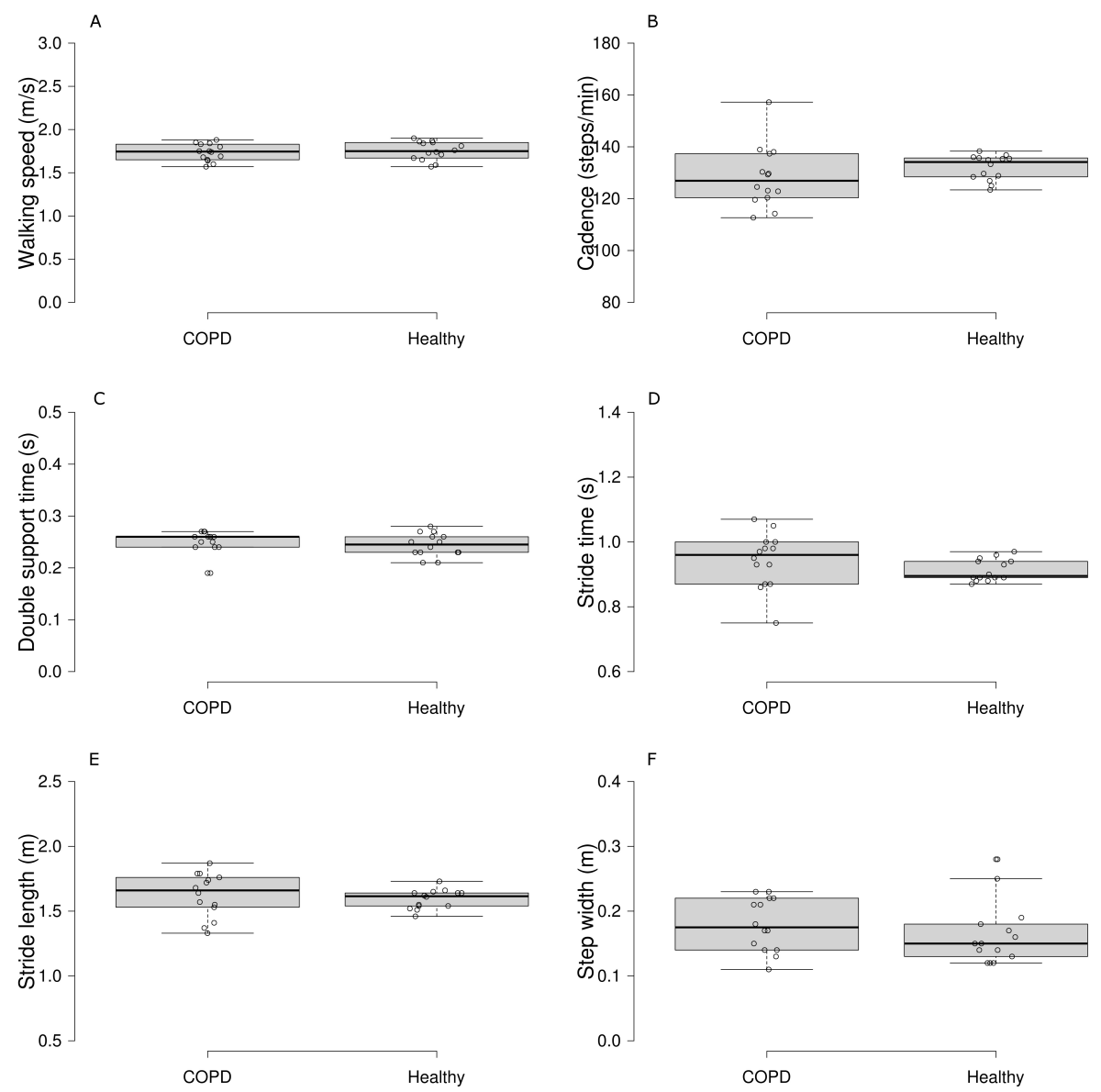

Figure 3.E.1. Boxplots of spatiotemporal gait characteristics in the sub-analysis. Patients with COPD $(n=14)$ and healthy elderly $(n=14)$ are depicted. Centre lines show the medians; box limits indicate the 25th and 75th percentiles; whiskers extend 1.5 times the interquartile range from the 25th and 75th percentiles, outliers are represented by dots. 
A

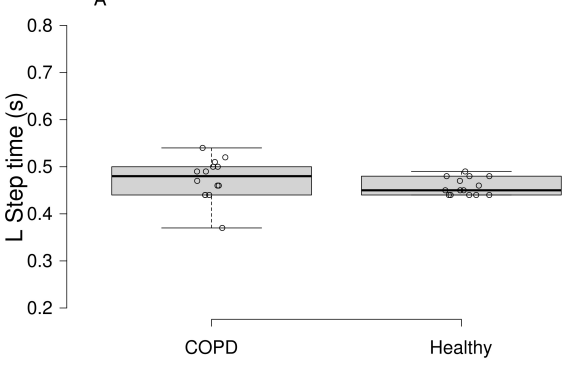

C

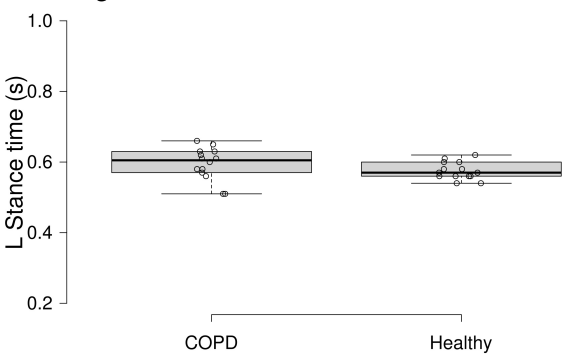

E

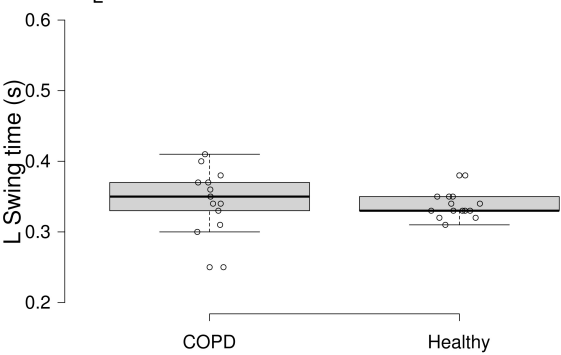

G

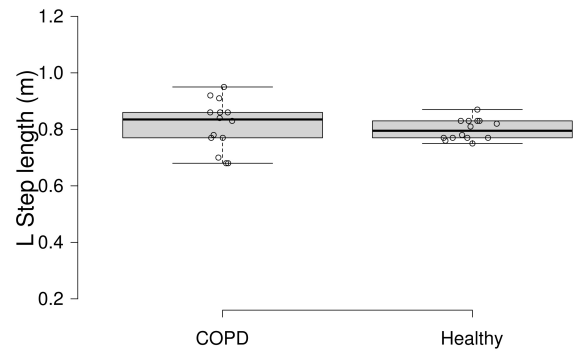

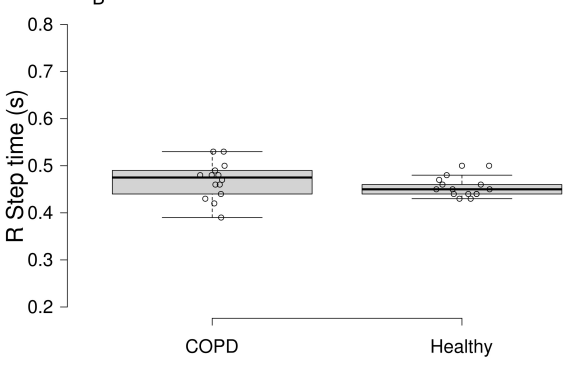

D

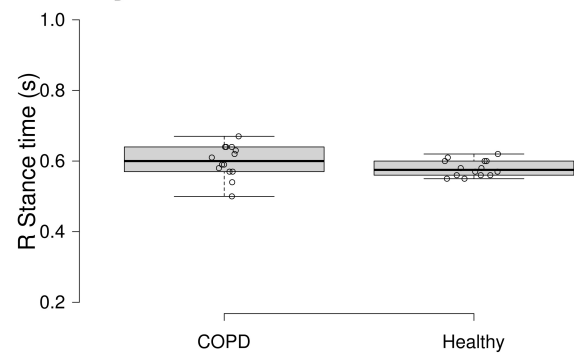

$\mathrm{F}$

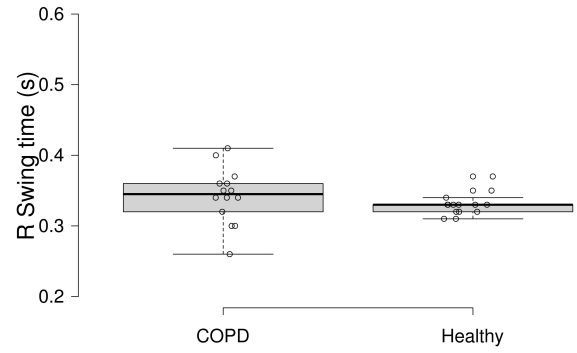

$\mathrm{H}$

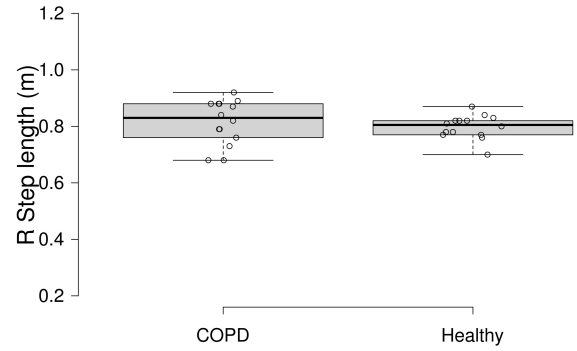

Figure 3.E.2. Boxplots of spatiotemporal gait characteristics separated for left and right side in the sub-analysis. Patients with COPD $(n=14)$ and healthy elderly $(n=14)$ are depicted. Centre lines show the medians; box limits indicate the 25th and 75th percentiles; whiskers extend 1.5 times the interquartile range from the 25 th and 75 th percentiles, outliers are represented by dots. 


\section{Bibliography}

1. Holland, A. E. et al. An official European Respiratory Society/American Thoracic Society technical standard: field walking tests in chronic respiratory disease. Eur Respir J 44, 1428-46 (2014).

2. Zeni J. A., J., Richards, J. G. \& Higginson, J. S. Two simple methods for determining gait events during treadmill and overground walking using kinematic data. Gait \& Posture 27, 710-4 (2008).

3. Ghoussayni, S. et al. Assessment and validation of a simple automated method for the detection of gait events and intervals. Gait Posture 20, 266-72 (2004).

4. Hansen, A. H., Childress, D. S. \& Meier, M. R. A simple method for determination of gait events. J Biomech 35, 135-8 (2002).

5. Hreljac, A. \& Marshall, R. N. Algorithms to determine event timing during normal walking using kinematic data. J Biomech 33, 783-6 (2000). 


\section{Alterations in stride-to-stride fluctuations in patients with chronic obstructive} pulmonary disease during the self-paced treadmill 6-minute walk test

Wai-Yan Liu, Martijn A. Spruit, Jeannet M. Delbressine, Paul J. Willems, Jennifer M. Yentes, Sjoerd M. Bruijn, Frits M.E. Franssen, Emiel F.M. Wouters, Kenneth Meijer.

Submitted for publication 


\section{Abstract}

Background The 6-minute walk test (6MWT) is regularly used for walking prescription for patients with chronic obstructive pulmonary disease (COPD) and can be administered validly on a self-paced treadmill, enabling gait assessment for improving gait function in COPD. This study aimed to assess stride-to-stride fluctuations in patients with COPD during a self-paced treadmill 6MWT compared to healthy elderly subjects.

Methods Eighty patients with COPD (mean age 62.3, SD 7.2 years; mean FEV 1 55.8, SD $19.4 \%$ predicted) and 39 healthy elderly subjects (mean age 62.1, SD 6.5 years; mean FEV 119.4, SD 17.0\% predicted) performed a self-paced, treadmill 6MWTs (Gait Real-time Analysis Interactive Lab), while spatiotemporal parameters and center of mass position were recorded (100 Hz, Vicon Nexus). Standard deviation, coefficient of variation (CoV), predictability (sample entropy) and local dynamic stability were calculated from 444 steps. Data were analyzed using ANCOVA with walking speed as a covariate.

Findings Patients with COPD walked slower than healthy elderly subjects, and demonstrated increased mean stride length $(p=0.033)$, increased standard deviation and CoV in stride length ( $p=0.005$ and $p=0.019$, respectively), and increased stride length predictability $(p=0.049)$.

Interpretation Patients with COPD demonstrate more variable stride lengths, while stride length patterns were more predictable than healthy elderly subjects during the 6MWT. These gait alterations may be associated with increased fall risk in patients with COPD. Further studies are recommended to identify the clinical value of gait assessment in COPD and its relation with falls in COPD. 


\section{Introduction}

Walking is one of the most problematic activities in daily life for patients with chronic obstructive pulmonary disease (COPD) [1]. Patients with COPD demonstrate gait alterations, including slower walking speed, shorter step lengths, increased time spent in double support, reduced step width and increased mediolateral trunk movements [2-4]. A better understanding of gait alterations in COPD and its relation to walking difficulties could provide targets for specific training programs and be beneficial for improving the overall health outcome in COPD.

The 6-minute walk test (6MWT) is an important measure of functional exercise capacity and prognosis, and evaluates response to treatment in patients with COPD [5]. In addition, the $6 \mathrm{MWT}$ is used to for health care decision-making $[6,7]$. This test is usually administered overground, which allows the subject to stop during the test. However, recently the 6MWT was shown to be valid on a self-paced treadmill [8]. Though the 6minute walk distance is the primary outcome of the 6MWT, gait assessment could be of added value for clinical purposes $[2,9,10]$.

The locomotor system integrates input from the motor cortex, cerebellum, basal ganglia and spinal cord, with feedback from visual, vestibular and proprioceptive sensors to control muscles and limbs [11]. In COPD, there are changes to the neuromuscular system [12, 13], proprioception [14-16], and brain function [17, 18]. The natural variations that occur from stride to stride while walking could be described as an output of the complex processing within the locomotor system. Furthermore, stride-to-stride fluctuations in gait of healthy individuals are characterized by a healthy amount of variability and predictability within the patterns of these fluctuations [19]. Alterations in stride-to-stride fluctuations may reflect a loss of flexible adaptations within the locomotor system and are associated with gait impairments within diseases $[20,21]$. This loss of flexible adaptations could be associated with serious accidents like falls.

Gait function can be assessed by calculating stride-to-stride fluctuations of spatiotemporal (time and distance) gait parameters and by evaluating the movement of the center of mass (COM) of the human body. The amount of fluctuations can be quantified by the standard deviation or coefficient of variation (CoV). However, this measure ignores the underlying patterns of stride-to-stride fluctuations over time [22]. Patterns over many gait cycles can be assessed using sample entropy [23] and local dynamic stability [24]. Sample entropy quantifies predictability of gait patterns [23]. Reduced sample entropy values indicate limitations in gait function, as has been found in patients with knee osteoarthritis [25]. Local dynamic stability assesses the stability of gait patterns. This measure serves as an early indicator for the risk of falling [24], and can differentiate healthy 
from unhealthy gait patterns in patients with peripheral arterial disease [21].

Alterations in stride-to-stride fluctuations are associated with fall risk in older adults [24, 26,27 ] and are therefore of interest in COPD, a population at increased risk for falls [15]. No differences in step time and step width predictability between patients with COPD and healthy subjects during fixed speed treadmill walking have been reported [4]. However, fixed speed treadmill walking interferes with natural stride-to-stride fluctuations in walking speed as compared to overground walking [28, 29]. Self-paced treadmill walking allows belt speed to fluctuate and may facilitate the assessment of stride-to-stride fluctuations that is more similar to overground walking [30]. Therefore, the aim of this study is to investigate whether altered stride-to-stride fluctuations are present in patients with COPD compared to healthy elderly subjects during a self-paced, treadmill 6MWT. It was hypothesized that patients with COPD show increased amount of variability in stride-tostride fluctuations, more predictable patterns of fluctuations, and reduced local dynamic stability of the center of mass patterns as compared to healthy elderly subjects.

\section{Methods}

\section{Participants}

The study recruited eighty patients with COPD (mean 62.3 SD 7.2 years; mean FEV 1 : 55.8 SD $19.4 \%$ predicted; 48 male, Table 4.1). As controls, thirty-nine healthy elderly subjects (mean 62.1 SD 6.5 years; mean $\mathrm{FEV}_{1}$ : 119.4 SD 17.0\% predicted; 25 male) were recruited. COPD was determined by spirometry using a post-bronchodilator $\mathrm{FEV}_{1} / \mathrm{FVC}$ cut-off ratio <0.7. Spirometry and electrocardiographic assessment were conducted prior to the 6MWT. Subjects with orthopedic ailments and/or neuromuscular diseases affecting their walking were excluded. Patients requiring walking aids or supplemental oxygen were excluded. Healthy elderly subjects with co-morbidities affecting the pulmonary and/or cardiovascular system were excluded. This study complied with the Declaration of Helsinki and was approved by the Medical research Ethics Committees United (MEC-U, M13-1374) in the Netherlands. All subjects gave informed consent prior to participation.

\section{Data collection}

Subjects walked on a split-belt instrumented treadmill placed in a virtual reality environment (Gait Real-time Analysis Interactive Lab, GRAIL, Motek Medical B.V., the Netherlands). Self-paced treadmill walking was enabled to regulate the belts' speed [10]. Reflective markers were placed on anatomical landmarks of each subject according to the 
Table 4.1. Demographics of sample subjects

\begin{tabular}{|c|c|c|c|c|}
\hline Parameter & & $\begin{array}{l}\text { COPD } \\
n=80\end{array}$ & $\begin{array}{l}\text { Healthy } \\
n=39\end{array}$ & $p$-value \\
\hline Age, years & & $62.3(7.2)$ & $62.1(6.5)$ & 0.878 \\
\hline Male, $n$ & & $48(60.0)$ & $25(64.1)$ & \\
\hline Weight, kg & & $75.9(16.9)$ & $79.1(12.8)$ & $0.301^{\#}$ \\
\hline Height, m & & $1.70(0.09)$ & $1.72(0.08)$ & $0.159^{\#}$ \\
\hline $\mathrm{BMI}, \mathrm{kg} / \mathrm{m}^{2}$ & & $26.3(5.1)$ & $26.8(3.1)$ & 0.499 \\
\hline $\mathrm{FEV}_{1} / \mathrm{FVC}$ & & $0.41(0.11)$ & $0.77(0.05)$ & $<0.001^{\#}$ \\
\hline $\mathrm{FEV}_{1} \%$ pred. & & $55.8(19.4)$ & $119.4(17.0)$ & $<0.001$ \\
\hline \multirow[t]{4}{*}{ GOLD group, $n$} & I & $10(12.5)$ & & \\
\hline & ॥ & $35(43.8)$ & & \\
\hline & III & $29(36.3)$ & & \\
\hline & IV & $6(7.5)$ & & \\
\hline Pack years, $n$ & & $41.2(19.2)$ & $8.3(12.1)$ & $<0.001^{\#}$ \\
\hline Never smoker, $n$ & & $1(1.3)$ & $15(38.5)$ & \\
\hline Former smoker, $n$ & & $73(91.3)$ & $23(59.0)$ & \\
\hline Current smoker, $n$ & & $6(7.5)$ & $1(2.6)$ & \\
\hline
\end{tabular}

Note: Data are presented as mean (SD), or if other specified as number (\%). Abbbreviations: COPD, chronic obstructive pulmonary disease; $\mathrm{BMI}$, body mass index; $\mathrm{FEV}_{1}$, forced expiratory volume in one second; FVC, forced vital capacity; GOLD, Global Initiative for Chronic Obstructive Disease.

\#: Non-parametric test.

Human Body Model (HBM1) of the lower limb [31]. Three-dimensional marker trajectories were recorded ( $100 \mathrm{~Hz}, 10$ Bonita cameras, Vicon Nexus, Oxford, UK). Treadmill force plate data were sampled at $1000 \mathrm{~Hz}$ in synchronization with the motion capture system.

Subjects performed one familiarization session of minimal 3 minutes. Then, two 6MWTs were performed. Patients performed the 6MWTs between pre pulmonary rehabilitation assessment and the first week of pulmonary rehabilitation. Healthy elderly subjects performed the 6MWTs within one day with minimal 45 minutes of rest in between the tests [10]. Perceived dyspnea and fatigue (Borg scale), heart rate and pulse oxygen saturation levels were assessed before and after each 6MWT. The instructions for the 6MWT were provided according to the European Respiratory Society/American Thoracic Society guidelines [5].

\section{Data analysis}

The 6MWT with the longest walk distance for each subject and without any stops was used for analysis. The first minute of the data (to minimize start-up effects) and the last 15 seconds (to minimize effects of deceleration of the treadmill) were excluded. Marker and force plate data were processed in custom MATLAB software (MathWorks Inc., Natick, USA) [10]. Gait events were calculated using a treadmill velocity based method and force 
plate data, as previously described [10]. Stride time (time from one heel contact to the next ipsilateral heel contact), stride length (distance between the toe marker and the ipsilateral toe marker at each heel contact in the anteroposterior direction, corrected for treadmill speed) and step width (distance between the toe marker in mediolateral direction between both feet at heel strike) were computed. The velocity of the center of mass $\left(\mathrm{CoM}_{\mathrm{vel}}\right)$ was computed using the position of the four pelvis markers. All subjects walked a minimum of 444 steps. Therefore, all time series were cut to 444 steps, as time series length affects the outcome parameters [23, 24].

\section{Stride-to-stride fluctuation analysis}

The standard deviation and CoV quantify the amount of variability in time series. The CoV was calculated as the standard deviation divided by the mean times 100 . The CoV was calculated over stride time, stride length and step width.

Sample entropy quantifies predictability and describes the probability that similar patterns in a time series are repeated. The method to compute this has been described previously [23]. A perfectly repeatable time series would reflect a sample entropy value of approximately 0 , while a completely random time series would reflect a sample entropy value extending towards infinity. The relative consistency of the group averages was examined for several combinations of input parameters of the tolerance radius $r$ and vector length $m$ [23]. The $r$ was chosen as $0.2^{*}$ standard deviation of the time series and $m$ was chosen as 2 for this study. In contrast to stride length and step width time series, stride time sample entropy seems to be a product of the parameter selection. Sample entropy values tended to direct to predictability in stride time with increasing $r$. Predictability of fluctuations in stride time itself could not be determined. Stride time sample entropy was therefore not included for statistical analysis [23].

Local dynamic stability is assessed by calculating the local divergence exponent, e.g. the exponential rate of divergence of adjacent trajectories in state space and has been described elsewhere [24]. A lower local divergence exponent reflects a more stable system. An embedding dimension of 6 was used for the mediolateral, 7 for the anteroposterior and 6 for the vertical direction. The $\mathrm{CoM}_{\mathrm{vel}}$ data were normalized to 100 samples in length per stride. A time delay of 10 samples was chosen as all the time series had the same frequency. The slope of the divergence curve provided an estimate of the local divergence exponent. The local divergence exponent was calculated over the $\mathrm{CoM}_{\mathrm{vel}}$ in three directions. To overcome non-stationarities, velocity time series was used to estimate local dynamic stability of the CoM patterns [24]. 
Table 4.2. The 6-minute walk test outcomes

\begin{tabular}{llll}
\hline Parameter & $\begin{array}{l}\text { COPD } \\
n=80\end{array}$ & $\begin{array}{l}\text { Healthy } \\
n=39\end{array}$ & $p$-value \\
\hline 6MWD, m & $496.7(79.3)$ & $691.1(64.4)$ & $<0.001$ \\
Walking speed, $\mathrm{m} / \mathrm{s}$ & $1.4(0.2)$ & $1.9(0.2)$ & $<0.001$ \\
Pre SpO $_{2}, \%$ & $95.2(1.5)$ & $97.0(1.0)$ & $<0.001^{\#}$ \\
Post $\mathrm{SpO}_{2}, \%$ & $92.3(4.6)$ & $97.1(1.4)$ & $<0.001^{\#}$ \\
Pre HR, bpm & $82.1(13.8)$ & $66.3(11.9)$ & $<0.001$ \\
Post HR, bpm & $103.0(18.1)$ & $98.8(22.5)$ & 0.309 \\
Pre dyspnea, score & $1.2(1.2)$ & $0.2(0.3)$ & $<0.001^{\#}$ \\
Post dyspnea, score & $4.7(2.3)$ & $1.1(1.0)$ & $<0.001^{\#}$ \\
Pre fatigue, score & $1.4(1.4)$ & $0.2(0.4)$ & $<0.001^{\#}$ \\
Post fatigue, score & $4.5(2.3)$ & $1.3(1.1)$ & $<0.001^{\#}$ \\
\hline
\end{tabular}

Note: Data are presented as mean (SD). Abbreviations: COPD, chronic obstructive pulmonary disease; $\mathrm{SpO}_{2}$, pulse oxygen saturation level; HR, heart rate; bpm, beats per minute.

\#: Non-parametric test.

\section{Statistics}

For demographic and clinical characteristics, either an independent T test or a Mann Whitney $U$ test was conducted to determine differences between the groups. Group comparisons were performed using an analysis of covariance (ANCOVA) with each gait parameter as dependent factor, group as independent factor, and walking speed as a covariate to correct for gait speed. A significance level of 0.05 was used. All statistical analyses were performed using SPSS 22.0 software (IBM Corp, Armonk, NY).

\section{Results}

Patients with COPD walked a shorter total distance than healthy elderly subjects and patients reported increased fatigue and dyspnea scores after the 6MWT (Table 4.2). Table 4.3 shows the spatiotemporal gait parameters and stride-to-stride fluctuations of gait parameters. Patients took larger stride lengths as compared to healthy elderly subjects $(F(1,116)=4.672, p=0.033)$. The standard deviation and CoV of stride length were increased in COPD as compared to healthy elderly subjects $(F(1,116)=8.089, p=0.005$ and $F(1,116)=5.658, p=0.019$, respectively). Patterns within stride length fluctuations were more predictable in patients with COPD as compared to healthy elderly subjects $(F(1,116)$ $=3.959, p=0.049$ ). No group differences were found for local dynamic stability of the $\mathrm{CoM}_{\text {vel }}$ patterns. The covariate, walking speed, was significantly related to mean stride time, mean stride length, standard deviation of stride time, standard deviation of step 
Table 4.3. Gait parameters of patients with COPD and healthy elderly subjects

\begin{tabular}{|c|c|c|c|c|}
\hline Parameter & $\begin{array}{l}\text { COPD } \\
n=80\end{array}$ & $\begin{array}{l}\text { Healthy } \\
n=39\end{array}$ & $\begin{array}{l}\text { Group } \\
\mathrm{F}, p\end{array}$ & $\begin{array}{l}\text { Speed } \\
\text { F, } p\end{array}$ \\
\hline Mean stride time, $\mathrm{s}$ & $1.02(0.09)$ & $0.89(0.05)$ & $0.618,0.434$ & $74.779,<0.001$ \\
\hline Mean stride length, $m$ & $1.43(0.18)$ & $1.73(0.14)$ & $4.672,0.033$ & $234.592,<0.001$ \\
\hline Mean step width, m & $0.18(0.04)$ & $0.17(0.05)$ & $1.513,0.221$ & $0.080,0.778$ \\
\hline SD stride time, $\mathrm{s}$ & $0.02(0.01)$ & $0.01(0.00)$ & $0.120,0.729$ & $18.541,<0.001$ \\
\hline SD stride length, $\mathrm{m}$ & $0.05(0.02)$ & $0.04(0.01)$ & $8.089,0.005$ & $2.134,0.147$ \\
\hline SD step width, m & $0.02(0.01)$ & $0.03(0.01)$ & $3.251,0.074$ & $15.884,<0.001$ \\
\hline CoV stride time, $\%$ & $1.96(0.77)$ & $1.64(0.32)$ & $0.273,0.602$ & $7.020,0.009$ \\
\hline CoV stride length, \% & $3.87(1.78)$ & $2.04(0.54)$ & $5.658,0.019$ & $21.396,<0.001$ \\
\hline CoV step width, \% & $13.97(5.35)$ & $17.51(8.32)$ & $0.028,0.868$ & $6.346,0.013$ \\
\hline SE stride length & $1.12(0.18)$ & $1.33(0.10)$ & $3.959,0.049$ & $7.353,0.008$ \\
\hline SE step width & $1.43(0.04)$ & $1.44(0.04)$ & $0.202,0.654$ & $0.984,0.323$ \\
\hline LDE CoM ${ }_{\text {vel }}-M L$ & $2.87(0.21)$ & $2.81(0.23)$ & $1.003,0.318$ & 6.647, 0.011 \\
\hline LDE CoMvel $-A P$ & $2.84(0.17)$ & $2.97(0.24)$ & $0.006,0.939$ & $6.844,0.010$ \\
\hline LDE CoMvel-V & $2.80(0.20)$ & $2.70(0.23)$ & $0.035,0.851$ & $2.976,0.087$ \\
\hline
\end{tabular}

Note: Data are presented as mean (SD). Abbreviations: COPD, chronic obstructive pulmonary disease; SD, standard deviation; CoV, coefficient of variation; SE, sample entropy; LDE, local divergence exponent; $C_{\text {M }} \mathrm{M}_{\text {vel }}$, center of mass velocity; $\mathrm{ML}$, mediolateral; $\mathrm{AP}$, anteroposterior; $\mathrm{V}$, vertical. All values were logarithmic transformed.

width, CoV of stride time, CoV of stride length, CoV of step width, predictability in stride length, and local divergence exponents of the $\mathrm{CoM}_{\mathrm{vel}}$ in mediolateral and anteroposterior direction $(p<0.05)$.

\section{Discussion}

The present study aimed to determine whether altered stride-to-stride fluctuations were present in patients with COPD compared to healthy elderly subjects during a self-paced treadmill 6MWT. We hypothesized that patients with COPD show increased amount of variability, increased predictability within the patterns of fluctuations, and less stability of movement patterns as compared to healthy elderly subjects. Our results demonstrated that patients with COPD achieved shorter walk distances than healthy elderly subjects, with increased amount of stride length variability and predictability of stride length patterns. These findings support the view that patients with COPD have a reduced gait function in comparison with healthy elderly subjects. In the current study however, local dynamic stability of the center of mass did not seem to be affected in patients with COPD. Increases in the amount of variability have been associated with reduced gait function. Increased amount of variability in gait parameters is associated with fall risk in the aging population [32, 33] and in pathological groups [34-36]. These changes may reflect less 
cooperative behavior between the different components of the locomotor system [19]. The present study showed an increased amount of stride-to-stride fluctuations in stride length in patients with COPD as compared to healthy elderly subjects. This is in line with earlier findings in step time in COPD [4].

Increased predictability (i.e. rigidity) of the locomotor system may be a sign of poor health $[19,21,37]$. Our patients displayed more predictable patterns of stride length fluctuations as compared to healthy elderly subjects. This finding reflects a lower rate of new information generation by the locomotor system and a more predictable behavior over time [23]. Our finding is in agreement with reduced entropy values in other physiological signals in COPD as compared to healthy individuals, including airflow and heart rate patterns [38].

Patients with COPD are characterized by alterations in gait [2-4, 10, 39-41] and diminished functioning [8]. However, the mechanisms for these changes are unclear and most probably multifactorial [42]. Skeletal muscle dysfunction, including muscle weakness and impaired muscle metabolism [13, 42], and fatigue [43] may be the reason for these impairments and could be associated with the alterations in stride-to-stride fluctuations in COPD. If true, the altered stride-to-stride fluctuations might be partially reversible following exercise-based pulmonary rehabilitation, which has shown to increase lower-limb muscle strength [44] and functional exercise capacity [5].

Falls in the aging population have been associated with stride-to-stride fluctuation measures $[24,33,45]$. Increased variability of stride lengths may increase the risk of falling during walking, for example, due to errors in foot placement and/or center of mass displacement [33]. Changes in the patterns within stride-to-stride fluctuations reflect the underlying mechanism of the locomotor system. Within the aging population, patients with COPD have a higher risk for falls [15]. Alterations in stride-to-stride fluctuations, both the amount and patterns of these fluctuations, are therefore of interest to identify the relationship between stride-to-stride fluctuation measures and fall risk in this specific patient population. Once this relationship is established, the coefficient of variation and sample entropy might be used as indicators for gait function and as targets for specific training programs.

In the current study however, patients with COPD did not demonstrate reduced local dynamic stability compared to healthy elderly subjects. This is in contrast to reduced local dynamic stability as found in the aging population [27]. A possible explanation could be that our patients were relatively in good condition and could have exhibited less gait and balance impairments, as they were able to perform the self-paced treadmill 6MWT without any stops or falls. Future studies are therefore recommended exploring stability measures in patients with COPD or exposing patients to different walking conditions, as 
this measure has been proposed as an indicator for fall risk [24, 27].

Several limitations within this study should be mentioned. The first limitation is that patients in GOLD 1 and GOLD 4 categories were less represented in this study. Patients classified into GOLD stage 4 were not able to perform the self-paced, treadmill 6MWT without any stops or using the handrails. Consequently, these patients were excluded for this study. Moreover, patients classified into GOLD stage 1 are less likely to be referred for pulmonary rehabilitation. The patients in this study do not reflect the heterogenic COPD patient population within all GOLD stages. The second limitation is that the effect of optic flow provided by the virtual reality environment was not examined in the specific patient or age group. This could have presented a challenge or helped those due to the increase in visual information during walking. However, a previous study assessed self-paced treadmill walking and virtual reality in healthy young adults [46]. The effects of virtual reality on gait were too small to be relevant and subjects reported walking with virtual reality as more similar to overground walking. The third limitation is the adaptation to self-paced treadmill walking. Subjects walked a minimum of 3 minutes to familiarize with self-paced treadmill walking. Due to the symptoms patients with COPD experience and their limited exercise capacity, they may not have been completely accustomed to adjust their walking speed. Though self-paced treadmill walking has been studied in healthy subjects [29], it has not been tested in this patient or age group.

In conclusion, patients with COPD demonstrate more variable stride lengths, while stride length patterns were more predictable as compared to healthy elderly subjects during the 6MWT. These gait alterations may be associated with increased fall risk in patients with COPD. Further studies are recommended to identify the clinical value of gait assessment in COPD and its relation with falls in COPD. 


\section{Bibliography}

1. Annegarn, J. et al. Problematic activities of daily life are weakly associated with clinical characteristics in COPD. J Am Med Dir Assoc 13, 284-90 (2012).

2. Annegarn, J. et al. Differences in walking pattern during 6-min walk test between patients with COPD and healthy subjects. PLoS One 7, e37329 (2012).

3. Nantsupawat, N. et al. Gait Characteristics in Patients With Chronic Obstructive Pulmonary Disease. J Prim Care Community Health 6, 222-6 (2015).

4. Yentes, J. M. et al. Patients with Chronic Obstructive Pulmonary Disease Walk with Altered Step Time and Step Width Variability as Compared with Healthy Control Subjects. Ann Am Thorac Soc 14, 858-866 (2017).

5. Holland, A. E. et al. An official European Respiratory Society/American Thoracic Society technical standard: field walking tests in chronic respiratory disease. Eur Respir J 44, 1428-46 (2014).

6. Jenkins, S. C. 6-Minute walk test in patients with COPD: clinical applications in pulmonary rehabilitation. Physiotherapy 93, 175-182 (2007).

7. Rodrigues, A. et al. Is the six-minute walk test a useful tool to prescribe high-intensity exercise in patients with chronic obstructive pulmonary disease? Heart Lung 45, 550-556 (2016).

8. Liu, W. Y. et al. Reproducibility and Validity of the 6-Minute Walk Test Using the Gait Real-Time Analysis Interactive Lab in Patients with COPD and Healthy Elderly. PLoS One 11, e0162444 (2016).

9. Liu, W. et al. Patients with COPD walk with less consistent organization of movement patterns of the lower extremity. Submitted.

10. Liu, W. et al. Spatiotemporal gait characteristics in patients with COPD during the Gait Real-time Analysis Interactive Lab-based 6-minute walk test. PLoS One 12, e0190099 (2017).

11. Hausdorff, J. M. Gait dynamics, fractals and falls: finding meaning in the stride-to-stride fluctuations of human walking. Hum Mov Sci 26, 555-89 (2007).

12. Gea, J. et al. Muscle dysfunction in chronic obstructive pulmonary disease: update on causes and biological findings. J Thorac Dis 7, E418-38 (2015).

13. Mathur, S., Brooks, D. \& Carvalho, C. R. Structural alterations of skeletal muscle in COPD. Front Physiol 5, 104 (2014).

14. Janssens, L. et al. Proprioceptive changes impair balance control in individuals with chronic obstructive pulmonary disease. PLoS One 8, e57949 (2013).

15. Roig, M. et al. Falls in people with chronic obstructive pulmonary disease: an observational cohort study. Respir Med 105, 461-9 (2011).

16. Voica, A. S. et al. Chronic obstructive pulmonary disease phenotypes and balance impairment. Int J Chron Obstruct Pulmon Dis 11, 919-25 (2016).

17. Alexandre, F. et al. Cortical implication in lower voluntary muscle force production in non-hypoxemic COPD patients. PLoS One 9, e100961 (2014).

18. Alexandre, F. et al. Brain Damage and Motor Cortex Impairment in Chronic Obstructive Pulmonary Disease: Implication of Nonrapid Eye Movement Sleep Desaturation. Sleep 39, 327-35 (2016).

19. Stergiou, N., Harbourne, R. \& Cavanaugh, J. Optimal movement variability: a new theoretical perspective for neurologic physical therapy. J Neurol Phys Ther 30, 120-9 (2006).

20. Hausdorff, J. M. et al. Is walking a random walk? Evidence for long-range correlations in stride interval of human gait. J Appl Physiol (1985) 78, 349-58 (1995).

21. Myers, S. A. et al. Gait variability is altered in patients with peripheral arterial disease. J Vasc Surg 49, 924-931 e1 (2009).

22. Dingwell, J. B. \& Cusumano, J. P. Nonlinear time series analysis of normal and pathological human walking. Chaos 10, 848-863 (2000).

23. Yentes, J. M. et al. The appropriate use of approximate entropy and sample entropy with short data sets. Ann Biomed Eng 41, 349-65 (2013). 
24. Bruijn, S. M. et al. Assessing the stability of human locomotion: a review of current measures. J R Soc Interface 10, 20120999 (2013).

25. Tochigi, Y. et al. Entropy analysis of tri-axial leg acceleration signal waveforms for measurement of decrease of physiological variability in human gait. J Orthop Res 30, 897-904 (2012).

26. Hausdorff, J. M. et al. Increased gait unsteadiness in community-dwelling elderly fallers. Arch Phys Med Rehabil 78, 278-83 (1997).

27. Toebes, M. J. et al. Local dynamic stability and variability of gait are associated with fall history in elderly subjects. Gait Posture 36, 527-31 (2012).

28. Dingwell, J. B. \& Cusumano, J. P. Re-interpreting detrended fluctuation analyses of stride-to-stride variability in human walking. Gait Posture 32, 348-53 (2010).

29. Sloot, L. H., van der Krogt, M. M. \& Harlaar, J. Self-paced versus fixed speed treadmill walking. Gait Posture 39, 478-84 (2014).

30. Wiens, C. et al. Walking speed and spatiotemporal step mean measures are reliable during feedbackcontrolled treadmill walking; however, spatiotemporal step variability is not reliable. J Biomech $\mathbf{8 3 , 2 2 1 - ~}$ 226 (2019).

31. Van den Bogert, A. J. et al. A real-time system for biomechanical analysis of human movement and muscle function. Med Biol Eng Comput 51, 1069-77 (2013).

32. Chamberlin, M. E. et al. Does fear of falling influence spatial and temporal gait parameters in elderly persons beyond changes associated with normal aging? J Gerontol A Biol Sci Med Sci 60, 1163-7 (2005).

33. Maki, B. E. Gait changes in older adults: predictors of falls or indicators of fear. J Am Geriatr Soc 45, 313-20 (1997).

34. Hausdorff, J. M. et al. Gait variability and basal ganglia disorders: stride-to-stride variations of gait cycle timing in Parkinson's disease and Huntington's disease. Mov Disord 13, 428-37 (1998).

35. Socie, M. J. et al. Gait variability and disability in multiple sclerosis. Gait Posture 38, $51-5$ (2013).

36. Webster, K. E., Merory, J. R. \& Wittwer, J. E. Gait variability in community dwelling adults with Alzheimer disease. Alzheimer Dis Assoc Disord 20, 37-40 (2006).

37. Lamoth, C. J. et al. Gait stability and variability measures show effects of impaired cognition and dual tasking in frail people. J Neuroeng Rehabil 8, 2 (2011).

38. Jin, Y. et al. Entropy change of biological dynamics in COPD. Int J Chron Obstruct Pulmon Dis 12, 29973005 (2017).

39. Lahousse, L. et al. Gait patterns in COPD: the Rotterdam Study. Eur Respir J 46, 88-95 (2015).

40. Yentes, J. M. et al. Walking abnormalities are associated with COPD: An investigation of the NHANES III dataset. Respir Med 105, 80-7 (2011).

41. Yentes, J. M. et al. Gait mechanics in patients with chronic obstructive pulmonary disease. Respir Res 16, 31 (2015).

42. Eisner, M. D. et al. COPD as a systemic disease: impact on physical functional limitations. Am J Med 121, 789-96 (2008).

43. Theander, K. et al. Severity of fatigue is related to functional limitation and health in patients with chronic obstructive pulmonary disease. Int J Nurs Pract 14, 455-62 (2008).

44. Spruit, M. A. et al. Resistance versus endurance training in patients with COPD and peripheral muscle weakness. Eur Respir J 19, 1072-8 (2002).

45. Callisaya, M. L. et al. Gait, gait variability and the risk of multiple incident falls in older people: a populationbased study. Age Ageing 40, 481-7 (2011).

46. Sloot, L. H., van der Krogt, M. M. \& Harlaar, J. Effects of adding a virtual reality environment to different modes of treadmill walking. Gait Posture 39, 939-45 (2014). 


\section{CHAPTER}

\section{Patients with COPD walk with less consistent organization of movement patterns of the lower extremity}

Wai-Yan Liu, Kendra K. Schmid, Kenneth Meijer, Martijn A. Spruit, Jennifer M. Yentes. Respiratory Care (in press). 


\section{Abstract}

Background The inherent stride-to-stride fluctuations during walking are altered in the aging population and could provide insight into gait impairments and falls in patients with chronic obstructive pulmonary disease (COPD). Stride-to-stride fluctuations are quantified two ways: 1) variability of the fluctuations (e.g. standard deviation) and 2) movement patterns within the fluctuations. Our objective was to investigate stride-to-stride fluctuations by evaluating the variability and movement patterns in lower limb joints of subjects with COPD compared to non-COPD, control subjects.

Methods In this cross-sectional study, 22 subjects with COPD (aged $63 \pm 9$ yrs.; FEV $154 \pm 19$ \%pred.) and 22 non-COPD control subjects (aged $62 \pm 9$ yrs.; FEV $195 \pm 18 \%$ pred.) walked for 3 minutes on a treadmill while their gait was recorded. The amount of variability (standard deviation and coefficient of variation) and movement patterns (predictability and consistency in organization) were quantified for the range of motion or joint angle of the hip, knee, and ankle, at three walking speeds (self-selected, FAST and SLOW). General linear mixed models were used for analysis.

Results Control subjects had a more consistent organization of the hip and knee joint movement patterns compared to subjects with $\operatorname{COPD}$ ( $p=0.015$ and $p=0.019$, respectively). Further, control subjects adapted to speed changes by demonstrating more consistent organization of movement patterns with faster speeds whereas subjects with COPD did not. Within the FAST walking speed, subjects with COPD demonstrated less consistent organization of knee and hip joint movement patterns as compared to non-COPD control subjects ( $p=0.025$ and $p=0.005$, respectively). Amount of variability did not differ between groups.

Conclusions Though subjects with COPD did not demonstrate decreased amount of variability, hip and knee joint movement patterns were less consistent in organization during walking. Reduced consistency in organization of movement patterns may be a contributing factor to falls and mobility problems experienced by subjects with COPD. 


\section{Introduction}

Chronic obstructive pulmonary disease (COPD) is characterized by progressive and persistent expiratory airflow limitations associated with chronic inflammation of the airway [1]. Patients with COPD are limited in their functional mobility and demonstrate lower limb muscle dysfunction [2, 3]. In addition, gait abnormalities have been found in patients with COPD, including loss of function at the ankle joint, slower cadence, shorter step length, and narrower step width [4-8].

Gait abnormalities can also be described by the inherent stride-to-stride fluctuations in gait parameters during walking [9]. Traditional methods quantify the variability, or the amount, of stride-to-stride fluctuations (e.g. the standard deviation or coefficient of variation). Complementary to traditional methods, other methods quantify movement patterns within the stride-to-stride fluctuations, such as how repeatable the fluctuations are within a walking bout. There are several methods available to quantify the movement patterns within stride-to-stride fluctuations. Sample entropy quantifies the predictability of the movement patterns, by measuring the probability that patterns will not be followed by additional similar patterns [10]. This method has been used to identify abnormal heart rate characteristics in COPD [11]. The local divergence exponent measures the consistency in the organization of movement patterns over time (referred to as local dynamic stability) [12]. Less consistent organization of movement patterns during walking have been found in fall-prone elderly subjects [13]. Quantifying movement patterns is important, as flexibility from stride-to-stride is needed to execute adaptations to the changing environment while walking [14]. Disease and aging have been associated with a loss of flexibility, reflected by either too rigid or too irregular movement patterns [15].

Stride-to-stride fluctuations have been studied scarcely in patients with COPD. A few studies have reported reduced variability in step width, and increased variability in step time and stride lengths in patients with COPD $[7,16]$. Similar gait changes have been related to increased fall risk in older adults $[17,18]$. However, these spatiotemporal gait variables provide only a snapshot of gait. Joint movements may provide a more global view of the lower extremity movements [7]. Previous studies describe altered lower limb joint movement patterns in older adults compared to young adults, and in patients with peripheral arterial disease compared to healthy subjects $[19,20]$. In addition, variability in spatiotemporal gait variables is not always discriminative between healthy older adults and older adults with a cautious gait [21]. Therefore, quantifying joint movement patterns in patients with COPD could provide additional insight into gait alterations and might explain increased balance disturbances, fall risk, and mobility problems in this population $[22,23]$. 
The aim of this study was to investigate stride-to-stride fluctuations by evaluating the variability and the movement patterns in lower limb joints while walking of subjects with COPD compared to non-COPD, control subjects. We hypothesized that subjects with COPD would demonstrate a more restricted and a less consistent organization of gait patterns compared to non-COPD control subjects, as reflected by decreased variability, increased predictability, and reduced consistency in organization in lower limb joint movement. To reveal adaptation needed to accommodate to alterations in demand, participants were also asked to walk outside of their comfortable walking speed.

\section{Material and methods}

\section{Participants}

In this cross-sectional study, subjects with COPD were recruited from the University of Nebraska Medical Center and the Omaha Veterans' Affairs Healthcare Center. COPD was previously diagnosed by a physician and confirmed by spirometry, using a ratio of forced expiratory volume in one second to forced vital capacity (FEV $1 / F V C)$ of less than 0.7 [1]. $\mathrm{FEV}_{1} \%$ predicted was calculated using the reference data source of the NHANES III survey [24]. Healthy control subjects were recruited through the community in Omaha and were considered healthy if they had an $\mathrm{FEV}_{1} / \mathrm{FVC}$ ratio above 0.7. Participants were excluded if they presented with a history of injury or disease that affected their mobility or another comorbidity that may affect the musculoskeletal, neurological, pulmonary, and/or cardiovascular systems. The Institutional Review Boards at both institutions approved the study. All participants provided written informed consent. In total, 22 subjects with COPD and 22 non-COPD control subjects were included (Table 5.1). This sample size was based upon a pilot study of stride-to-stride fluctuations of step length and step time in subjects with COPD prior to the current study.

\section{Data collection}

All participants wore a tight-fitting suit (i.e., wrestling singlet). Retroreflective markers were placed on bony landmarks of the body, bilaterally, according to a modified Helen Hayes marker set [25]. Prior to data collection, participants were asked to walk on a treadmill in order to determine their self-selected walking speed (SSWS). The procedure to assess the SSWS comprised of a walking trial in which if a subject indicated that a speed was comfortable, the subject continued to walk at that speed for 1 minute and then asked again if the speed was too fast or too slow. If the subject indicated it was too fast, the treadmill was slowed or vice versa. This continued until a comfortable speed was 
Table 5.1. Demographics of subjects used for analysis

\begin{tabular}{llll}
\hline Parameter & $\begin{array}{l}\text { Control } \\
n=22\end{array}$ & $\begin{array}{l}\text { COPD } \\
n=22\end{array}$ & $p$-value \\
\hline Age, years & $62.1(8.6)$ & $62.7(9.0)$ & 0.825 \\
Male/Female, $n$ & $6 / 16$ & $13 / 9$ & $0.033^{\dagger}$ \\
FEV $_{1}, \mathrm{~L}$ & $2.4(0.6)$ & $1.8(0.8)$ & $0.011^{*}$ \\
FVC, L & $3.1(0.7)$ & $3.2(1.1)$ & 0.703 \\
FEV $1 /$ FVC & $0.78(0.06)$ & $0.55(0.12)$ & $<0.001^{*}$ \\
FEV $_{1} \%$ pred. & $94.5(17.7)$ & $53.7(18.5)$ & $<0.001^{*}$ \\
Height, cm $_{\text {Weight, kg }}$ & $165.0(9.4)$ & $172.5(13.3)$ & $0.038^{*}$ \\
BMI, kg/m & $73.6(15.6)$ & $94.5(30.7)$ & $0.007^{*}$ \\
SLOW, m/s & $27.0(4.9)$ & $31.6(8.7)$ & $0.049^{*}$ \\
SSWS, m/s & $0.75(0.13)$ & $0.68(0.21)$ & 0.230 \\
FAST, m/s & $0.93(0.16)$ & $0.85(0.26)$ & 0.230 \\
\hline
\end{tabular}

Note: Data are presented as mean (SD) or as frequencies. Abbreviations: $\mathrm{FEV}_{1}$, forced expiratory volume in 1 second; FVC, forced vital capacity; BMI, body mass index; SSWS, self-selected walking speed.

*: Significant difference between groups.

$\dagger$ : An association was found between gender and group $\left(X^{2}(1)=4.539, p=0.033\right)$.

found, which was similar to the participant's normal pace. This procedure may have taken up to fifteen minutes, in which subjects also became accustomed to treadmill walking itself. The SSWS corresponded to the participant's normal pace, for example, when they would walk from the car into a building. Then, participants were given a minimum fiveminute rest to prevent fatigue. Participants completed 3.5-minute trials at their SSWS, in which data were recorded during the last three minutes. Participants completed two additional trials at $-20 \%$ (SLOW) and $+20 \%$ (FAST) of their SSWS. The order of the latter two walking speeds was randomized for all participants. Participants rested a minimum of two minutes to recover between trials.

Three-dimensional marker trajectories were collected with a high-speed motion capture system (Motion Analysis Corp., Santa Rosa, CA; $60 \mathrm{~Hz}$ ) while participants walked on a treadmill (Figure 5.1). Unfiltered three-dimensional marker data were used to calculate sagittal joint angle time series for the ankle, knee, and hip (Visual 3D, C-Motion, Inc., Germantown, MD). The range of motion (RoM) was calculated for every right and left step from the joint angle time series using custom Matlab program (Mathworks, Inc., Natick, MA) (Figure 5.2). Generated time series for RoM data was cut to 162 steps. This was based upon the participant with the lowest number of steps of all trials. To quantify the variability of fluctuations, standard deviations, and coefficients of variation were calculated from the RoM time series. To quantify the movement patterns, RoM time series were subjected to predictability analysis (see description below). In addition, the right leg 


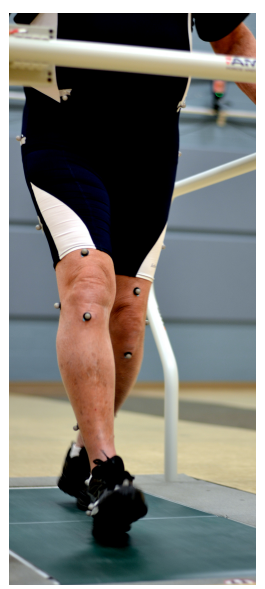

Figure 5.1. Gait assessment setup. Notes: Infrared cameras are placed throughout the laboratory. Calibrated cameras triangulate the position of each marker based on the reflection of infrared light back to the camera lens off the retroflective marker. The positions of markers were used to calculate joint angles and range of motion of the joints.

joint angles from the ankle, knee and hip were subjected to consistency of organization analysis (see description below).

Sample entropy was used to assess the predictability of the RoM time series. The method to compute sample entropy has been described elsewhere [10]. Briefly, sample entropy compares patterns within the time series to determine the probability that the same pattern will not appear again in the time series. A sample entropy value near zero reflected a near perfect predictable time series of RoM values, whereas, a sample entropy value extending toward infinity reflected a completely random time series of RoM values. A decrease in predictability has possible associations with a loss of flexible adaptation. Sample entropy was calculated using the $m$ parameter, the number of data points that are to be compared $(m=2)$, the $r$ parameter, the similarity criterion $(r=0.25)$ and the $N$ parameter, the length of the entire data set $(\mathrm{N}=162)$, on the RoM time series for each joint (see Supplementary Material 5.A for justification of parameter selection) [10].

Lower limb joint angles were subjected to consistency of organization analysis (i.e. local dynamic stability) [26]. To describe this analysis, imagine drawing a circle on a piece of paper over and over in a continuous fashion. Likely each iteration of the circle does not perfectly overlap the last circle. The further apart each iteration is, the less consistent the path is over time; whereas, if each iteration closely mimicked the iteration before, the path would be considered highly consistent. Similarly, this is true of the movement of the joint in space. Briefly, the joint angle time series was reconstructed into a multidi- 

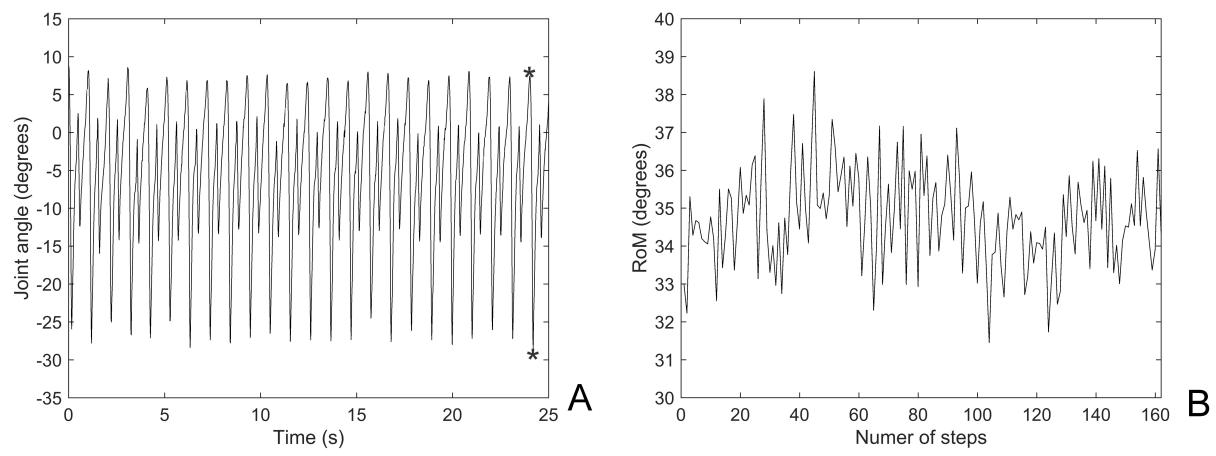

Figure 5.2. A graphic representation of the joint angle and range of motion time series of the right ankle of a healthy subject. Notes: A) Represents a selection of the joint angle time series of the ankle (0-25 seconds). The asterisks reflect the maximum and minimum value of the joint angle in one stride, (e.g. the range of motion in one stride; RoM). B) Represents the RoM time series calculated over the entire joint angle time series.

mensional space and the distances between these points were calculated as a function of time and averaged over all data point pairs to obtain the average logarithmic rate of divergence (Figure 5.3). The slope of the divergence curve provided an estimate of the local divergence exponent, which quantifies the separation of the joint angle trajectories over time. A higher value of the local divergence exponent indicates a less consistent organization of movement pattern [27].

Demographics were compared using a t-test or a chi square test. A linear mixed model was used to assess differences in mean, standard deviation and coefficient of variation, and predictability of RoM time series between groups (COPD vs. controls) and over the three walking speed conditions (SLOW, SSWS, and FAST). A linear mixed model was used to determine group and speed differences of the consistency of organization in each joint angle (ankle, knee, hip). This method enables the determination of differences within and between groups while accounting for correlation due to repeated measurements and adjusting for potentially confounding variables. All interactions between group and speed were investigated. Adjustments for multiple comparisons were made using the simulation technique. In all models, the actual speed at each (SLOW, SSWS and FAST) was used as a covariate so comparisons could be made between speed groups independent of the starting speed. Mean differences (MDs) between groups or speeds, $95 \%$ confidence intervals (Cls), and effect sizes (Cohen's d)[30] were calculated. All statistical analyses were conducted in SAS (SAS institute, Inc., Cary, NC). A significance level of 0.05 was set a priori. 

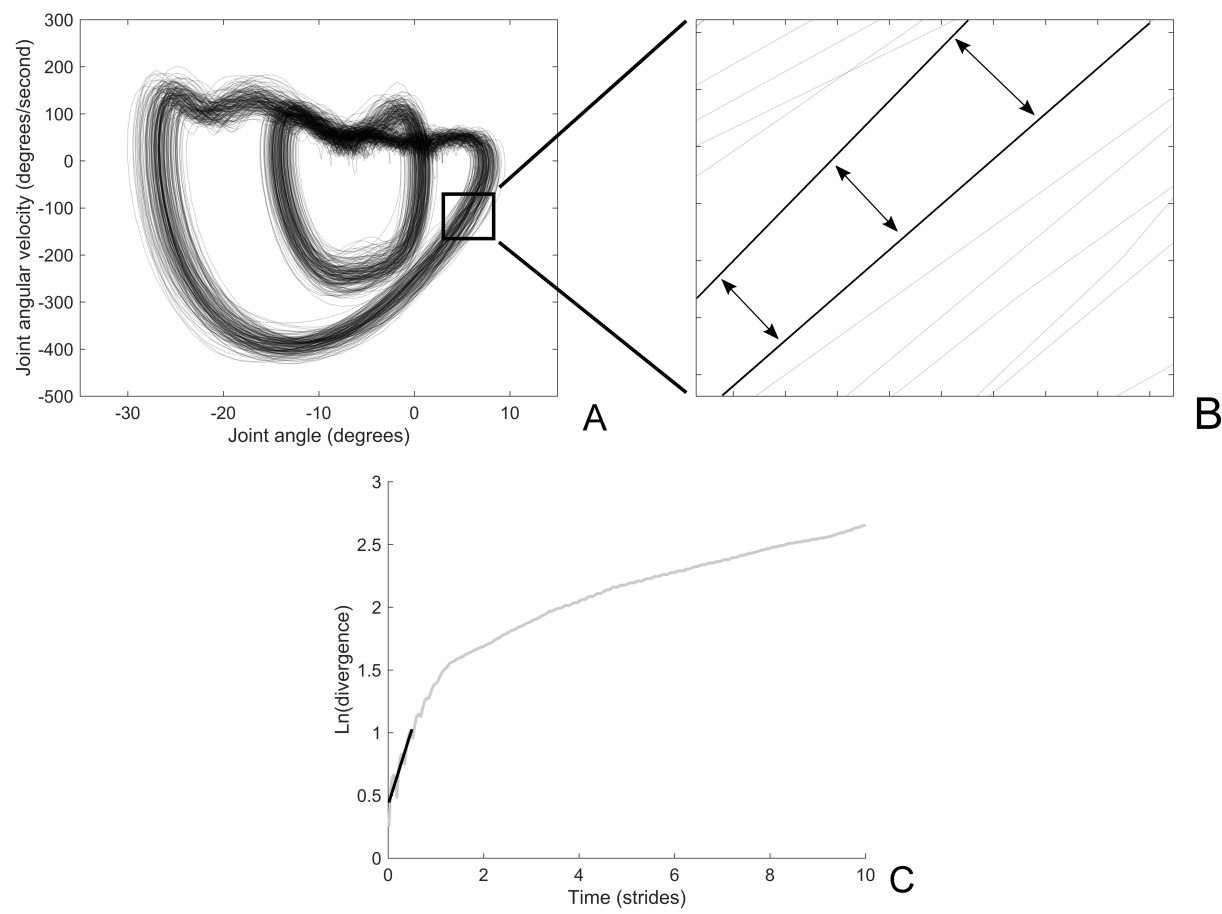

Figure 5.3. A graphic representation shows the ankle joint angle time series of a healthy subject in two dimensions and the calculation of the local divergence exponent (figure adapted from [12, 20]). Notes: A) The phase portrait of a representative ankle joint angle (angle vs. angular velocity). B) A close-up view of one portion of the phase portrait where the divergence of neighboring trajectories is outlined. Distances between points were calculated as a function of time. C) The local divergence exponent is calculated as the slope of the strides vs. the natural log of the divergence plot at 0 0.5 strides (grey line). To reconstruct the angles in a multi-dimensional space, time delay of 10 points[28] and embedding dimension (ankle and knee: 7; hip: 6 dimensions)[29] were calculated and used. Each joint angle time series was normalized to 100 data points per stride.

\section{Results}

\section{Variability of fluctuations}

A significant interaction between group and speed for mean ankle RoM and mean hip RoM was found ( $p=0.016$ and $p=0.035$, respectively, see Figure 5.4 and Figure 5.5 , and Supplementary Material 5.B for additional information). COPD and control subjects seem to have a similar ankle and hip RoM at slow speed, however, only control subjects seem to increase their RoM with faster speeds.

Knee RoM standard deviation demonstrated a main effect of speed for both groups com- 

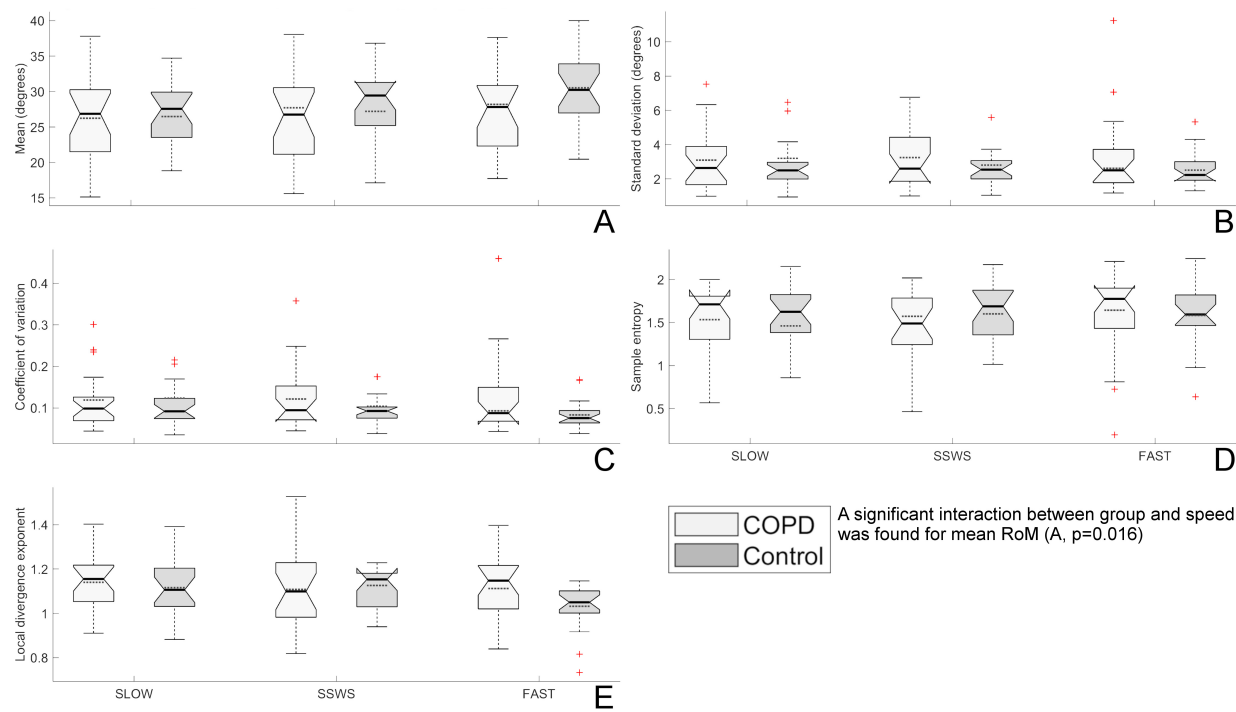

Figure 5.4. Boxplots of the gait parameters of the ankle joint between subjects with COPD (light grey) and control subjects (dark grey). A) Mean ankle range of motion; B) standard deviation of the ankle range of motion; C) coefficient of variation of the ankle range of motion; D) predictability of the ankle range of motion; E) consistency in the organization of the ankle joint angle. Note: Centerlines show the medians, dotted centerlines show the means; box limits indicate the 25th and 75th percentiles; whiskers extend to the most extreme data points not considered outliers; outliers are plotted using the ' + ' symbol.

bined ( $p=0.015$, see Figure 5.6). Knee RoM was more variable in the FAST condition compared to SSWS ( $p=0.01$, see Table 5.2) and SLOW condition ( $p=0.049$, Table 5.2).

Similar to standard deviation, knee RoM coefficient of variation demonstrated a significant main effect of speed $(p=0.010)$. Walking at the FAST speed was more variable in knee RoM compared to SSWS $(p=0.01, d=0.64)$ and SLOW speed $(p=0.02)$. A main effect of speed on hip RoM coefficient of variation was found ( $p=0.036)$. Walking at the FAST speed was more variable in hip RoM compared to SSWS $(p=0.046)$ and SLOW speed $(p=0.033)$.

\section{Movement patterns within the fluctuations}

No main effect of group was found for predictability across any of the joints. A main effect of speed for hip RoM predictability was found ( $p=0.016)$. During the FAST condition, hip RoM was more predictable compared to SSWS $(p=0.0495)$ and SLOW speed $(p=0.01)$. No interactions were found. 
Table 5.2. Significant adjusted post-hoc analyses

\begin{tabular}{|c|c|c|c|c|c|}
\hline Parameter & Comparison & $\begin{array}{l}\text { Mean } \\
\text { difference }\end{array}$ & $95 \% \mathrm{Cl}$ & $\begin{array}{l}\text { Effect } \\
\text { size }\end{array}$ & $p$-value \\
\hline \multirow{2}{*}{$\begin{array}{l}\text { Standard deviation } \\
\text { of knee RoM }\end{array}$} & FAST vs. SSWS & 0.40 & $0.08-0.72$ & 0.63 & $0.010^{*}$ \\
\hline & FAST vs. SLOW & 0.46 & $0.00-0.92$ & 0.51 & $0.049^{*}$ \\
\hline \multirow{2}{*}{$\begin{array}{l}\text { Coefficient of variation } \\
\text { of knee RoM }\end{array}$} & FAST vs. SSWS & 0.01 & $0.00-0.02$ & 0.64 & $0.010^{*}$ \\
\hline & FAST vs. SLOW & 0.01 & $0.00-0.03$ & 0.57 & $0.022^{*}$ \\
\hline \multirow{2}{*}{$\begin{array}{l}\text { Coefficient of } \\
\text { variation of hip RoM }\end{array}$} & FAST vs. SSWS & 0.01 & $0.00-0.01$ & 0.51 & $0.046^{*}$ \\
\hline & FAST vs. SLOW & 0.01 & $0.00-0.02$ & 0.54 & $0.033^{*}$ \\
\hline \multirow[t]{2}{*}{ Predictability of hip RoM } & FAST vs. SSWS & -0.11 & $-0.23-0.00$ & 0.50 & 0.0495 \\
\hline & FAST vs. SLOW & -0.20 & $-0.37--0.04$ & 0.62 & $0.010^{*}$ \\
\hline $\begin{array}{l}\text { Consistency in organization } \\
\text { of knee joint angles }\end{array}$ & $\begin{array}{l}\text { COPD vs. } \\
\text { controls at FAST }\end{array}$ & 0.11 & $0.01-0.21$ & 0.91 & $0.025^{*}$ \\
\hline $\begin{array}{l}\text { Consistency in organization } \\
\text { of hip joint angles }\end{array}$ & $\begin{array}{l}\text { COPD vs. } \\
\text { controls at FAST }\end{array}$ & 0.15 & $0.03-0.28$ & 1.07 & $0.005^{*}$ \\
\hline $\begin{array}{l}\text { Consistency in organization } \\
\text { of hip joint angles in COPD }\end{array}$ & FAST vs. SSWS & 0.10 & $0.00-0.19$ & 0.85 & $0.045^{*}$ \\
\hline $\begin{array}{l}\text { Consistency in organization } \\
\text { of hip joint angles in COPD }\end{array}$ & FAST vs. SLOW & 0.11 & $0.00-0.23$ & 0.85 & $0.045^{*}$ \\
\hline
\end{tabular}

Note: Data are presented as mean difference. Abbreviations: RoM, range of motion; SSWS, self-selected walking speed; COPD, chronic obstructive pulmonary disease; Cl Confidence Interval.

${ }^{*}$ : Significant difference between conditions or groups. 

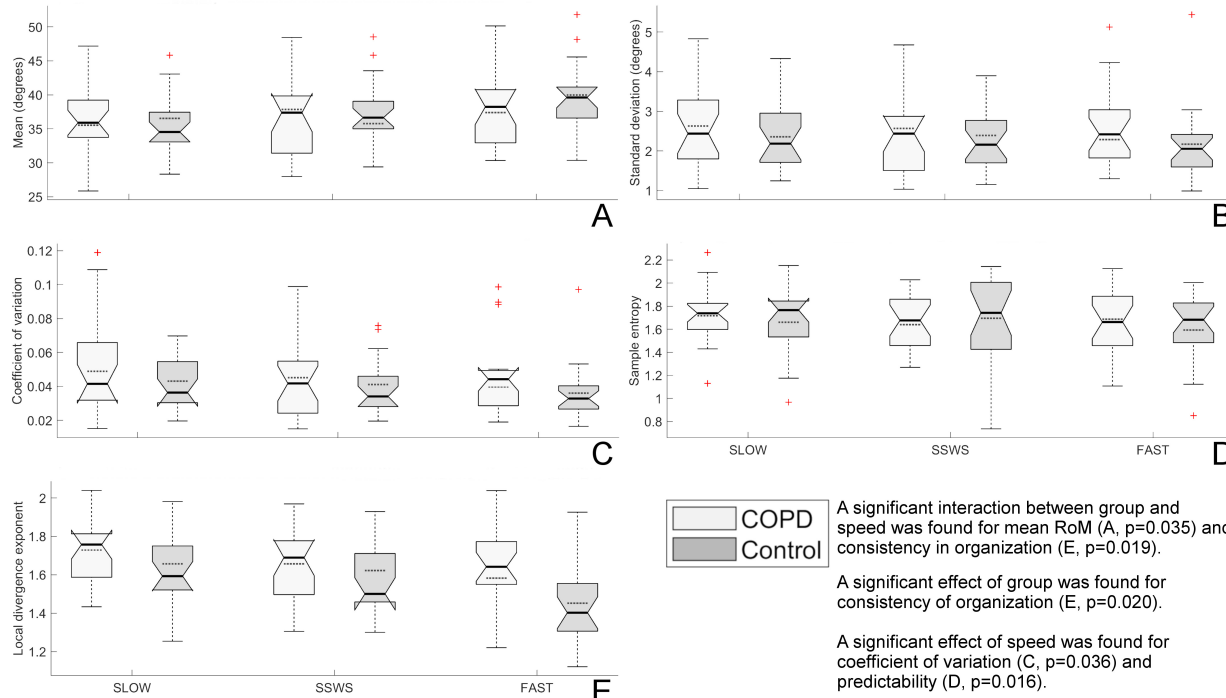

1 COPD A significant interaction between group and

speed was found for mean RoM $(A, p=0.035)$ and

Control consistency in organization $(E, p=0.019)$

A significant effect of group was found for consistency of organization $(E, p=0.020)$.

A significant effect of speed was found for coefficient of variation $(C, p=0.036)$ and predictability $(D, p=0.016)$.

Figure 5.5. Boxplots of the gait parameters of the hip joint between subjects with COPD (light grey) and control subjects (dark grey). A) Mean hip range of motion; B) standard deviation of the hip range of motion; C) coefficient of variation of the hip range of motion; D) predictability of the hip range of motion; E) consistency in the organization of the hip joint angle. Note: Centerlines show the medians, dotted centerlines show the means; box limits indicate the 25th and 75th percentiles; whiskers extend to the most extreme data points not considered outliers; outliers are plotted using the ' + ' symbol.

A significant interaction between group and speed was found in the consistency in the organization of the knee $(p=0.015)$ and hip $(p=0.019)$ joint angle. Non-COPD, control subjects increased consistency in organization (e.g. lower local divergence exponent values) in the hip and knee angle movement patterns as speed increased, while subjects with COPD did not demonstrate as much improvements. In the FAST condition, subjects with COPD showed significantly reduced consistency in organization of the knee joint angle as compared to non-COPD, control subjects $(p=0.025)$ and for the hip joint angle $(p=0.005)$. Within the COPD group, hip joint angles demonstrated reduced consistency in organization (e.g. greater local divergence exponent values) in the FAST condition as compared to the SSWS and SLOW speed ( $p=0.045$ and $p=0.045$, respectively). 

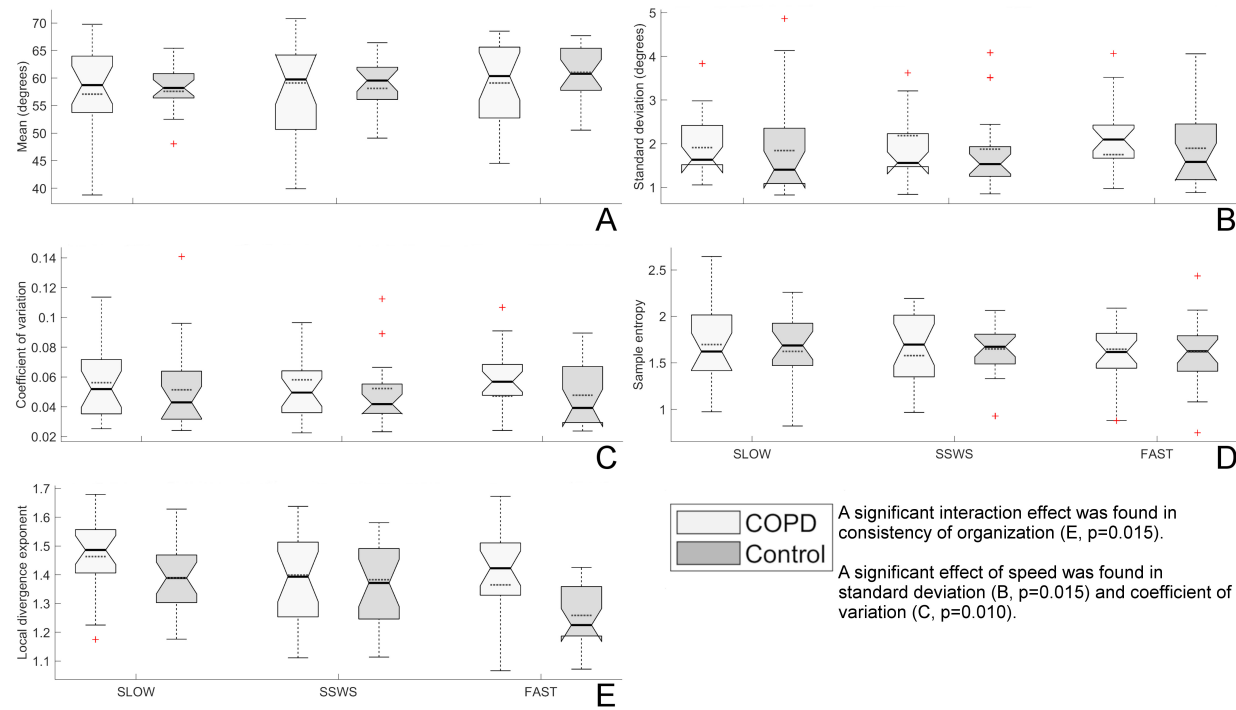

COPD A significant interaction effect was found in Control

consistency of organization $(E, p=0.015)$.

A significant effect of speed was found in standard deviation $(B, p=0.015)$ and coefficient of

Figure 5.6. Boxplots of the gait parameters of the knee joint between subjects with COPD (light grey) and control subjects (dark grey). A) Mean knee range of motion; B) standard deviation of the knee range of motion; C) coefficient of variation of the knee range of motion; D) predictability of the knee range of motion; E) consistency in the organization of the knee joint angle. Note: Centerlines show the medians, dotted centerlines show the means; box limits indicate the 25th and 75th percentiles; whiskers extend to the most extreme data points not considered outliers; outliers are plotted using the ' + ' symbol.

\section{Discussion}

The present study aimed to investigate stride-to-stride fluctuations by evaluating the variability and movement patterns in lower limb joints while walking of subjects with COPD as compared to non-COPD, control subjects. We hypothesized that subjects with COPD would demonstrate more restricted and less consistent organization of movement patterns, as reflected by a reduced variability of fluctuations, increased predictability, and reduced consistency in the organization of joint movement patterns within the stride-tostride fluctuations. In addition, it was hypothesized that this would become more apparent at walking speeds that differed from the SSWS.

Data partially supported our hypothesis. In agreement, subjects with COPD demonstrated less consistent organization of the knee and hip joint angles as compared to control subjects. However, control subjects adapted to speed changes by demonstrating more consistent organization of movement patterns with faster speeds than subjects with COPD did. During FAST walking, subjects with COPD, compared to controls, showed less con- 
sistency in the organization of both knee and hip joint angles, suggesting that gait instability impairments in the locomotor system becomes apparent when accommodating to increase in task demands. In contrast to our hypothesis, no differences between groups were found for the variability of fluctuations, nor predictability.

Healthy joint angle movement patterns represent a coordinated locomotor system able to make flexible adaptations to demands placed on the body during walking [9]. Subjects with COPD might have been unable to select the required response when forced to accommodate to alterations in task demands (e.g. a change from SSWS) [20]. Reduced consistency in the organization of joint movement patterns suggests the presence of neuromuscular deterioration in the locomotor system, reflecting less healthy movement patterns [20]. Reduced consistency in the organization of movement patterns during walking are present in subjects with peripheral arterial disease [20] and fall-prone elderly subjects [13].

In those with COPD, reduced consistency in the organization of the joint movement patterns could be related to extrapulmonary manifestations, such as skeletal muscle dysfunction present in COPD [2]. A loss of muscle mass and strength in the lower limb muscles has been found in subjects with COPD, including the quadriceps, the tibialis anterior and gastrocnemius [31, 32]. Alterations in these skeletal muscles are especially important because these muscles are essential for walking and adjusting to task and environmental demands. Therefore, reduced strength in the lower limb muscles could be associated with gait alterations and its stride-to-stride fluctuations [4-7, 16]. In addition, the lack of ability in subjects with COPD to adjust the movement patterns with changes in walking speed, may be associated with an increased susceptibility for balance disturbances and falls present in this patient population [22, 23].

Subjects in the current study did not demonstrate a more restricted gait, as reflected by a reduced variability of fluctuations, as compared to control subjects. A possible explanation could be that variability is a measure of magnitude and may not be sensitive enough to changes in fluctuations between groups, unless these changes are drastic or have a lot of outliers [33]. Our results showed that subjects with COPD did not differ in predictability of movement patterns from control subjects, whereas the consistency in the organization of movement patterns was different between the groups. A possible explanation could be that predictability and consistency measures quantify different aspects of movement patterns; both related to the flexibility of the locomotor system as found in healthy subjects. In addition, conflicting results have been reported on sample entropy in COPD. Yentes et al. [7] reported that predictability in step time and step width was not sensitive to differentiate subjects with COPD from non-COPD subjects. However, differences between patients and healthy subjects were reported in heart rate and airflow 
patterns [11,34]. Less consistency in the organization of movement patterns was found in various patient populations as compared to healthy older adults $[12,13,20]$, however this measure has not been applied in subjects with COPD. As existing knowledge of variability of and patterns within stride-to-stride fluctuations in subjects with COPD is limited, these measures should be used to complement each other to gain further insight into gait alterations in COPD.

The patterns of the joint movement pattern within the strides differed between the groups, as measured by the consistency in the organization of movement patterns. There are two likely explanations for these differences: 1 ) biomechanical limits of the joint movement and 2) the nature of the data that was used in each method. First, the anatomy of the human body limits the RoM. Consequently, the RoM is less variable due to the anatomical limits of the minimum and maximum that a joint can move. However, within the movement from minimum to maximum, the joint angle can be variable and fluctuate, and may provide information about the joint movement pattern that the RoM does not take into account. Second, only continuous joint angle data demonstrated differences between groups as compared to the discrete, RoM data. Discrete time series (i.e. time series of distinct events within the gait cycle) are more appropriate for the calculation of sample entropy. Therefore, RoM time series was used for this analysis [14]. The consistency in organization analysis was calculated using the continuous data of joint angles, as this is more traditional for this measure. Consequently, consistency measures in joint angle time series may be more appropriate to differentiate gait alterations in COPD from controls subjects. Further research is warranted to identify appropriate measures to assess movement patterns in COPD and the clinical importance of these measures.

The present study supports the effect of walking speed on both the variability and movement patterns of stride-to-stride fluctuations [35-41]. Variability of fluctuations in the knee and hip joint RoM, was greatest at the FAST walking speeds in both groups; that is, variability was greater in the FAST speed compared to both the SWSS and SLOW speeds. However, no consistent relationship was found for the variability of stride-to-stride fluctuations and walking speed, as studies reported a negative linear relationship [35, 39] or a U-shaped relationship [37, 41]. One study by Kang and Dingwell [42] reported, though not statically significant, a trend of higher mean standard deviations in knee and hip flexionextension, and ankle plantarflexion-dorsiflexion, in older adults with increasing walking speed (from preferred walking speed to $+20 \%$ of preferred walking speed). This is similar to the changes in RoM observed in present study. A possible explanation could be that faster walking speeds poses increased difficulty for the subject, which resulted in increased variability at FAST walking speed. Similar to variability, walking speed alters movement patterns within stride-to-stride fluctuations [36-40]. Our findings showed in- 
creased predictability of the hip RoM during FAST walking, while Costa et al. [36] found highest predictability values during walking at a normal speed. A possible explanation for these differences may lie in the difference between overground, as used by Costa et al. [36], and treadmill walking, as used in the present study. In addition, previous studies reported reduced consistency in the organization of gait movement patterns with increasing walking speeds $[38,40]$. This contrasts with our findings, as no clear trend was found in the consistency in the organization of the joint angles over the walking speeds. However, differences in walking speed might have affected these results, as our participants walked at their SSWS, whereas one other study based the walking speed on fast walking determined during the 6-minute walk test [40], while another study scaled the walking speed to the leg length and pendulum dynamics [38].

This study has some limitations. The use of $\mathrm{FEV}_{1} / \mathrm{FVC}$ ratio of $<0.70$ as confirmatory of airflow obstruction may misclassify some individuals in the current study. However, all subjects in the COPD group also had a physician diagnosis of COPD. In addition, a potential effect of the male to female ratio, height, weight and body mass index on the study results should be mentioned, as differences were found between COPD and control subjects. In the current study, we did not record if subjects with COPD took a bronchodilator prior to their visit to our research facility. In addition, subjects with COPD have a reduced walking capacity due to the pathophysiology of the disease. The duration of treadmill walking is therefore limited, resulting in shorter data lengths per trial in which the biological complexity of the movement pattern could not be captured. However, sample entropy and local divergence exponents are quite robust against different data lengths and tend to respond better to short data lengths $[10,43]$. Another limitation is the speed perturbations of $20 \%$ of the participant's SSWS may not have been challenging enough. Therefore, measures to assess the amount of variability and patterns within the stride-to-stride fluctuations to identify gait impairments may not have surfaced in subjects with COPD. In addition, treadmill walking differs from overground walking. The treadmill might have opposed a constraint on the participants by limiting the fluctuations in walking that are normally present in overground walking [44]. Little differences in joint angles have been reported between treadmill and overground walking [45]. However, it is possible that the stride-to-stride fluctuations of the data were affected by the use of the treadmill, as differences in stride-to-stride fluctuations in spatiotemporal gait parameters have been reported between overground and treadmill walking [16, 46-48]. Another limitation is the potentially heterogeneous sample of subjects with COPD in this study. The present study included a sample size of 44 ( 22 subjects per group). Therefore, further studies involving a larger population are suggested to determine additional parameters for gait impairments in subjects with COPD. Contractile fatigue occurs during exercise in some subjects with COPD and may be a limiting factor in exercise tolerance [3]. Subjects that 
experience exercise limitations by fatigue may have presented more gait impairments as compared to subjects who are mainly limited by dyspnea. However, dyspnea and fatigue were not assessed in the present study. Future studies are therefore recommended to investigate the association between the heterogeneity in COPD and gait impairments. Furthermore, the observations could not be related to COPD itself, as body composition changes and differences in muscle mass of lower extremities may have affected the stride-to-stride fluctuations in subjects with COPD and control subjects. Body composition and muscle strength of the lower extremities have not been assessed in the current study. Research in the contribution of body composition and muscle strength are therefore recommended for future studies.

\section{Conclusions}

Though subjects with COPD did not demonstrate decreased amount of variability, subjects with COPD show reduced consistency in the organization of hip and knee joint angle patterns across the walking speeds. Reduced consistency in the organization of the hip and knee joint movements might be a contributing factor to falls and mobility problems experienced by subjects with COPD. 


\section{Bibliography}

1. Web Page. Accessed: 02-02-2019. https : / / www . goldCOPD . org (Global Initiative for Chronic Obstructive Lung Disease).

2. Mathur, S., Brooks, D. \& Carvalho, C. R. Structural alterations of skeletal muscle in COPD. Front Physiol 5, 104 (2014).

3. Saey, D. et al. Contractile leg fatigue after cycle exercise: a factor limiting exercise in patients with chronic obstructive pulmonary disease. Am J Respir Crit Care Med 168, 425-30 (2003).

4. Annegarn, J. et al. Differences in walking pattern during 6-min walk test between patients with COPD and healthy subjects. PLoS One 7, e37329 (2012).

5. Lahousse, L. et al. Gait patterns in COPD: the Rotterdam Study. Eur Respir J 46, 88-95 (2015).

6. Nantsupawat, N. et al. Gait Characteristics in Patients With Chronic Obstructive Pulmonary Disease. J Prim Care Community Health 6, 222-6 (2015).

7. Yentes, J. M. et al. Patients with Chronic Obstructive Pulmonary Disease Walk with Altered Step Time and Step Width Variability as Compared with Healthy Control Subjects. Ann Am Thorac Soc 14, 858-866 (2017).

8. Yentes, J. M. et al. Gait mechanics in patients with chronic obstructive pulmonary disease. Respir Res 16, 31 (2015).

9. Stergiou, N. et al. Nonlinear Tools in Human Movement (Human Kinetics Publishers, Inc., Champaign, IL, 2004).

10. Yentes, J. M. et al. The appropriate use of approximate entropy and sample entropy with short data sets. Ann Biomed Eng 41, 349-65 (2013).

11. Goulart, C. D. L. et al. Respiratory muscle strength effect on linear and nonlinear heart rate variability parameters in COPD patients. Int J Chron Obstruct Pulmon Dis 11, 1671-1677 (2016).

12. Bruijn, S. M. et al. Assessing the stability of human locomotion: a review of current measures. $J R$ Soc Interface 10, 20120999 (2013).

13. Lockhart, T. E. \& Liu, J. Differentiating fall-prone and healthy adults using local dynamic stability. Ergonomics 51, 1860-72 (2008).

14. Stergiou, N., Harbourne, R. \& Cavanaugh, J. Optimal movement variability: a new theoretical perspective for neurologic physical therapy. J Neurol Phys Ther 30, 120-9 (2006).

15. Vaillancourt, D. E. \& Newell, K. M. Changing complexity in human behavior and physiology through aging and disease. Neurobiol Aging 23, 1-11 (2002).

16. Liu, W. Y. et al. Spatiotemporal gait characteristics in patients with COPD during the Gait Real-time Analysis Interactive Lab-based 6-minute walk test. PLoS One 12, e0190099 (2017).

17. Brach, J. S. et al. Too much or too little step width variability is associated with a fall history in older persons who walk at or near normal gait speed. J Neuroeng Rehabil 2, 21 (2005).

18. Callisaya, M. L. et al. Gait, gait variability and the risk of multiple incident falls in older people: a populationbased study. Age Ageing 40, 481-7 (2011).

19. Buzzi, U. H. et al. Nonlinear dynamics indicates aging affects variability during gait. Clin Biomech (Bristol, Avon) 18, 435-43 (2003).

20. Myers, S. A. et al. Gait variability is altered in patients with peripheral arterial disease. J Vasc Surg 49, 924-931 e1 (2009).

21. Herman, T. et al. Gait instability and fractal dynamics of older adults with a "cautious" gait: why do certain older adults walk fearfully? Gait \& Posture 21, 178-85 (2005).

22. Crisan, A. F. et al. Balance impairment in patients with COPD. PLoS One 10, e0120573 (2015).

23. Roig, M. et al. Falls in people with chronic obstructive pulmonary disease: an observational cohort study. Respir Med 105, 461-9 (2011).

24. Hankinson, J. L., Odencrantz, J. R. \& Fedan, K. B. Spirometric reference values from a sample of the general U.S. population. Am J Respir Crit Care Med 159, 179-87 (1999). 
25. Houck, J. R., Duncan, A. \& De Haven, K. E. Knee and hip angle and moment adaptations during cutting tasks in subjects with anterior cruciate ligament deficiency classified as noncopers. J Orthop Sports Phys Ther 35, 531-40 (2005).

26. Bruijn, S. M. et al. Maximum Lyapunov exponents as predictors of global gait stability: a modelling approach. Med Eng Phys 34, 428-36 (2012).

27. Stergiou, N. Nonlinear Analysis for Human Movement Variability (CRC Press, Boca Raton, 2016).

28. Thomas, R. D. et al. An efficient algorithm for the computation of average mutual information: Validation and implementation in Matlab. J Math Psychol 61, 45-59 (2014).

29. Kennel, M. B., Brown, R. \& Abarbanel, H. D. Determining embedding dimension for phase-space reconstruction using a geometrical construction. Phys Rev A 45, 3403-3411 (1992).

30. Cohen, J. Statistical Power Analysis for the Behavioral Sciences (Routledge, New York, 1988).

31. Donaldson, A. V. et al. Muscle function in COPD: a complex interplay. Int J Chron Obstruct Pulmon Dis 7 , 523-35 (2012).

32. Cabaleiro-Lago, C., Nilsson, M. \& Soderman, O. Self-diffusion NMR studies of the host-guest interaction between beta-cyclodextrin and alkyltrimethylammonium bromide surfactants. Langmuir 21, 11637-44 (2005).

33. Stergiou, N. \& Decker, L. M. Human movement variability, nonlinear dynamics, and pathology: is there a connection? Hum Mov Sci 30, 869-88 (2011).

34. Dames, K. K., Lopes, A. J. \& de Melo, P. L. Airflow pattern complexity during resting breathing in patients with COPD: effect of airway obstruction. Respir Physiol Neurobiol 192, 39-47 (2014).

35. Chien, J. H. et al. The Effect of Walking Speed on Gait Variability in Healthy Young, Middle-aged and Elderly Individuals. J Phys Act Nutr Rehabil 2015 (2015)

36. Costa, M. et al. Multiscale entropy analysis of human gait dynamics. Physica A 330, 53-60 (2003).

37. Dingwell, J. B. \& Marin, L. C. Kinematic variability and local dynamic stability of upper body motions when walking at different speeds. J Biomech 39, 444-52 (2006).

38. England, S. A. \& Granata, K. P. The influence of gait speed on local dynamic stability of walking. Gait Posture 25, 172-8 (2007).

39. Jordan, K., Challis, J. H. \& Newell, K. M. Walking speed influences on gait cycle variability. Gait \& Posture 26, 128-34 (2007).

40. Manor, B., Wolenski, P. \& Li, L. Faster walking speeds increase local instability among people with peripheral neuropathy. J Biomech 41, 2787-92 (2008).

41. Sekiya, N. et al. Optimal walking in terms of variability in step length. J Orthop Sports Phys Ther 26, 26672 (1997).

42. Kang, H. G. \& Dingwell, J. B. Separating the effects of age and walking speed on gait variability. Gait Posture 27, 572-7 (2008).

43. Dingwell, J. B. \& Cusumano, J. P. Nonlinear time series analysis of normal and pathological human walking. Chaos 10, 848-863 (2000).

44. Parvataneni, K. et al. Kinematic, kinetic and metabolic parameters of treadmill versus overground walking in healthy older adults. Clin Biomech (Bristol, Avon) 24, 95-100 (2009).

45. Riley, P. O. et al. A kinematic and kinetic comparison of overground and treadmill walking in healthy subjects. Gait \& Posture 26, 17-24 (2007).

46. Dingwell, J. B. et al. Local dynamic stability versus kinematic variability of continuous overground and treadmill walking. J Biomech Eng 123, 27-32 (2001).

47. Hollman, J. H. et al. A comparison of variability in spatiotemporal gait parameters between treadmill and overground walking conditions. Gait Posture 43, 204-9 (2016).

48. Terrier, P. \& Deriaz, O. Kinematic variability, fractal dynamics and local dynamic stability of treadmill walking. J Neuroeng Rehabil 8, 12 (2011). 


\section{Supplementary Material}

\section{Parameter selection for sample entropy}

The relative consistency of the group averages were examined for several combinations of parameters, $r$ (the similarity criterion) and $m$ (length of the data segment being compared), see Figure 5.A.1, as proposed by Yentes et al. [1]. For the present study, $r$ was chosen as $0.25 \times$ standard deviation of the time series, as this parameter was the most consistent, and an $m$ of 2 was chosen. 

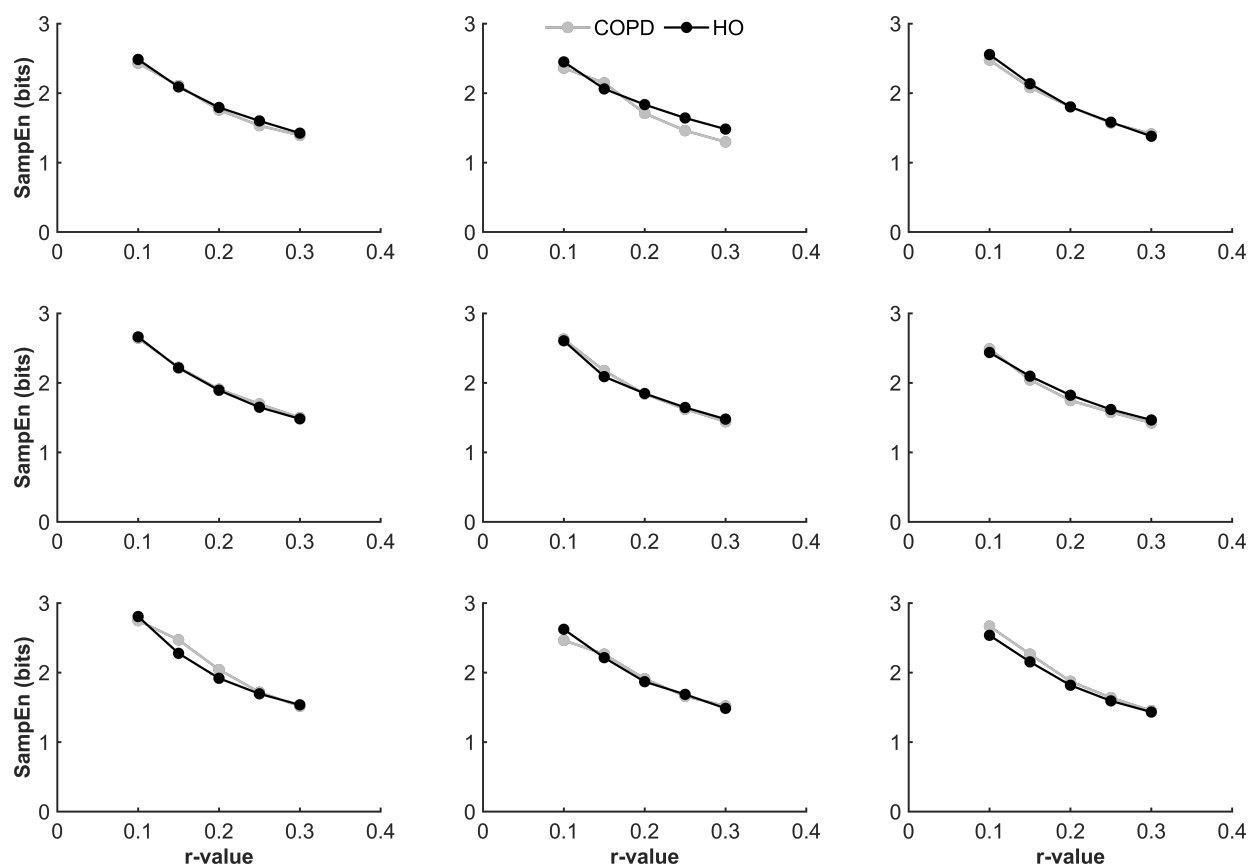

Figure 5.A.1. Sample entropy as a function of tolerance level $(r)$ when $m=2$. The left panel reflects the SLOW walking speed, the middle panel reflects the self-selected walking speed and the right panel reflects the FAST walking speed. The top reflects the ankle range of motion, the middle reflects the knee range of motion and the bottom reflects the hip range of motion. SampEn: sample entropy. 


\section{Stride-to-stride fluctuations between groups}

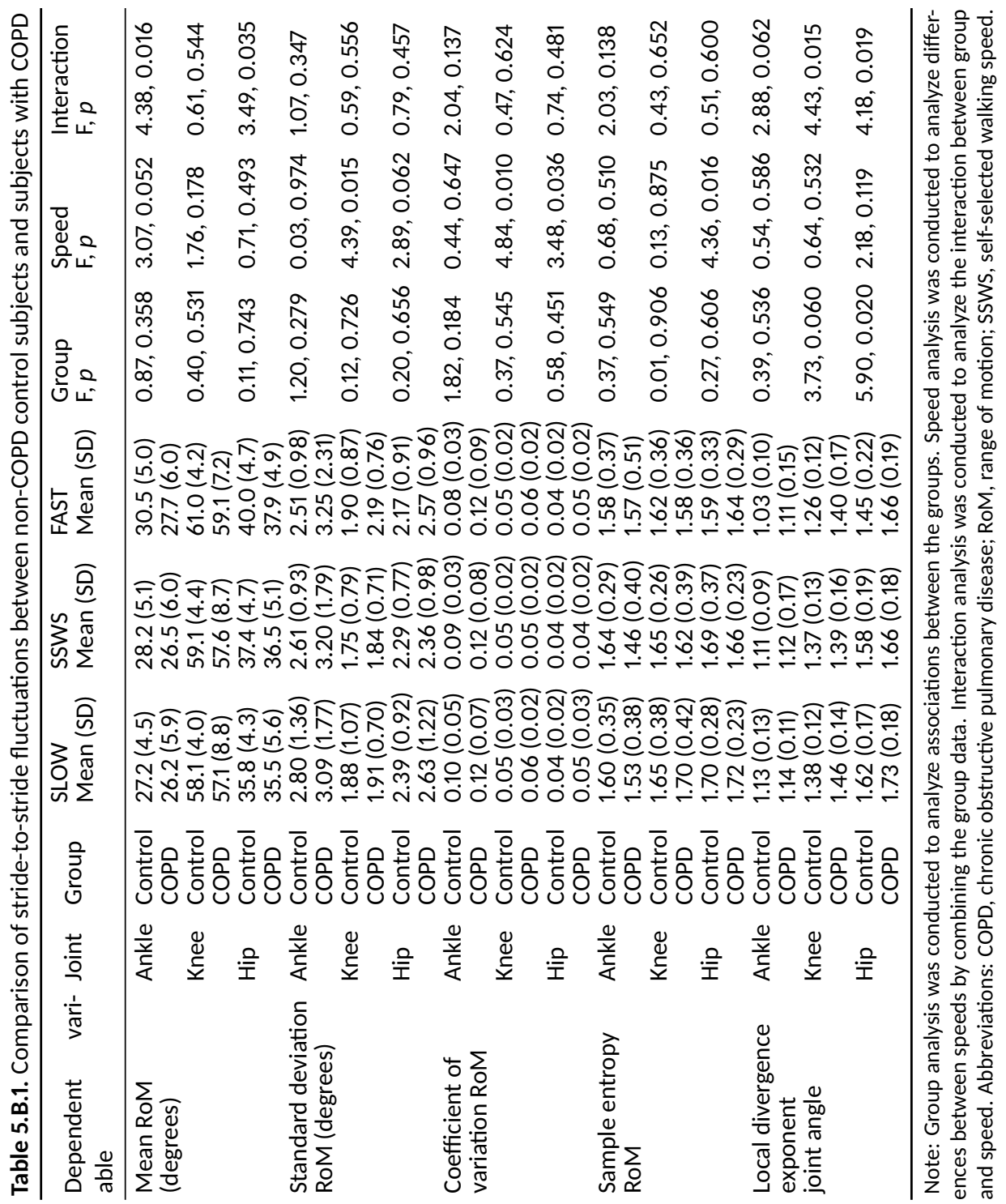




\section{Bibliography}

1. Yentes, J. M. et al. The appropriate use of approximate entropy and sample entropy with short data sets. Ann Biomed Eng 41, 349-65 (2013). 


\section{CHAPTER}

\section{Effects of pulmonary rehabilitation on gait characteristics in patients with COPD}

Wai-Yan Liu, Kenneth Meijer, Jeannet M. Delbressine, Paul J. Willems, Emiel F.M. Wouters, Martijn A. Spruit.

Journal of Clinical Medicine. 2019;8(4),459. 


\section{Abstract}

Background and aim Pulmonary rehabilitation (PR) improves lower-limb muscle function in patients with chronic obstructive pulmonary disease (COPD). However, it remains unclear whether patients improve gait characteristics, in particular stride-to-stride fluctuations that are associated with fall risk. This study aims to identify whether and to what extent PR affects positively gait characteristics in COPD.

Methods In this prospective observational study, 44 COPD patients (aged: $62 \pm 7$ years; $\mathrm{FEV}_{1}: 56 \pm 20 \%$ predicted) performed self-paced, treadmill 6-minute walk tests (Gait Real-time Analysis Interactive Lab) before and after PR, while spatiotemporal parameters and center of mass position were recorded (100 Hz, Vicon Nexus). Standard deviation, coefficient of variation, predictability (sample entropy), and consistency in organization (local divergence exponent) were calculated. Sub-analysis was performed to identify gait differences between good and poor responders ( $<30 \mathrm{~m}$ change in 6-minute walk distance).

Results Patients demonstrated shorter stride times ( $p=0.001)$ and improved lower-limb muscle function $(p<0.001)$ following PR. The good responders had a greater increase in stride length $(p<0.001)$ and a greater decrease in stride time $(p<0.001)$ compared to the poor responders.

Discussion/Conclusion Current PR improved stride time in patients, while movement patterns within stride-to-stride fluctuations did not change. Training programs specifically targeting balance issues and gait function may be beneficial in improving gait characteristics in COPD. 


\section{Introduction}

Walking has been reported as a problematic activity in daily life in patients with chronic obstructive pulmonary disease (COPD) [1,2]. Patients with COPD are characterized by gait alterations as compared to healthy older adults [3-7]. These gait alterations, in particular changes in the inherent stride-to-stride fluctuations, have been associated with noncommunicable diseases [8, 9], and falls in the aging population [10-13]. Moreover, strideto-stride fluctuations may represent the capability of the locomotor system to make flexible adaptations during walking, characterized by a healthy amount of, and patterns within these fluctuations [14]. Alterations in the amount and patterns of stride-to-stride fluctuations, whether too rigid or too random, are associated with aging, disease and falls [6, 9, $10,15]$. In addition, gait deficits have been suggested to be an intrinsic risk factor for fall risk in COPD [16], indicating the clinical value of gait assessment in COPD. Improved insight into gait deficits in patients with COPD may direct future training programs focusing on gait function.

Gait function has been associated with muscle capacity in older adults, suggesting a link between lower-limb muscle weakness and altered gait $[17,18]$. Exercise training has a beneficial effect on gait characteristics in older adults, including increased walking speed, cadence, stride length and shorter stride times [19]. Lower-limb muscle dysfunction and impaired exercise capacity are often reported in $\operatorname{COPD}[3,20]$. However, pulmonary rehabilitation (PR) has proven to be an effective intervention for improving lower-limb muscle function and exercise capacity in patients with COPD [21-23]. Consequently, PR may have beneficial effects on gait characteristics in patients with COPD. However, whether and to what extent PR improves gait characteristics in patients with COPD is currently unknown. In addition, improvements in gait characteristics may differ between patients with COPD as their responses to PR are differential [24, 25].

Within PR for COPD, the 6-minute walk test (6MWT) is used to evaluate response to treatment [26]. It has been shown that the 6-minute walk distance (6MWD) and gait characteristics can be obtained during a self-paced, treadmill 6MWT in COPD [4, 27]. In the present study, we aimed to identify whether a comprehensive PR program affects gait characteristics in patients with COPD during a self-paced, treadmill 6MWT. Patients with COPD are able to walk faster during the 6MWT after PR [26]. Consequently, it is hypothesized that stride length will increase, whereas stride time and step width will decrease. In addition, we hypothesized that the amount of stride-to-stride fluctuations will decrease, and the patterns within stride-to-stride fluctuations will be less predictable and more consistent in the organization after PR, resulting in improved movement patterns. Response to PR is heterogeneous in COPD, we therefore hypothesized that good responders will demon- 
strate more improvements in gait characteristics as compared to poor responders.

\section{Experimental section}

\section{Study population}

This study included 44 patients with COPD who were referred for a PR program in the specialized rehabilitation center CIRO in Horn, the Netherlands. COPD was diagnosed according to the GOLD guidelines, including a post-bronchodilator spirometry (MasterScreen Body, Carefusion, Houten, the Netherlands) [28]. Patients with COPD were eligible if they were between 40-85 years of age and clinically stable evaluated by the physician. In addition, electrocardiography was conducted during pre-rehabilitation assessment and evaluated by the physician. Patients were excluded when they presented other lung diseases (e.g. asthma, lung cancer, sarcoidosis, tuberculosis or lung fibrosis), neuromuscular or orthopedic ailments affecting their gait, and/or if they used supplemental oxygen and/or walking aids. The study was approved by the Medical Research Ethics Committees United, the Netherlands (M13-1374) and registered in the Dutch Trial Registers (NTR4421). This study complied with the Declaration of Helsinki. All patients gave written informed consent prior to participating this study.

\section{Study design}

This was a prospective, observational trial. Patients underwent a routine 3-day pre rehabilitation assessment [29], in which patients performed, amongst other things, a maximal incremental cycle test during which peak work rate was determined. Patients performed a constant work rate test at $75 \%$ of the determined peak work rate and regular 6MWTs. In addition, a careful characterization of the extra-pulmonary features and comorbidities of patients with COPD were performed, which determined the application of various treatments: physical exercise training, occupational therapy, dietary counselling, psychosocial counselling, education and exacerbation management.

The pre-rehabilitation assessment was followed by a PR program in line with the American Thoracic Society/European Respiratory Society Statement on PR [23]. PR consisted of 40 sessions that could be offered inpatient ( 8 weeks for 5 days/week) or outpatient ( 8 weeks for 3 days/week; followed by 8 weeks for 2 days/week). Physical exercise training was the cornerstone of the PR program, comprising of exercises to strengthen muscle groups in the upper and lower extremities, treadmill walking and stationary cycling. All exercises were performed at moderate-to-high intensity to obtain an overload training 
stimulus, conform the FITT principles as described by the American College of Sports Medicine [30]. The training intensity increased during the PR period, based on dyspnea and fatigue symptom scores. In addition, all patients underwent flexibility exercises, general physical exercise for lower and upper extremities, and daily supervised 30-min outdoor walks [25]. The program was implemented by an interdisciplinary team including a chest physician, respiratory nurse, dietician, occupational therapist, physiotherapist, psychologist, and social worker.

Finally, a 2-day routine outcome assessment was conducted to evaluate the effects of the PR program for each patient. The response of PR on functional exercise capacity was assessed using the overground 6MWT [26]. Functional mobility was assessed using the Timed Up and Go test (TUG test) [31]. Body composition was assessed using dual energy X-ray absorptiometry (Prodigy, DEXAtech Benelux B.V., Ridderkerk, the Netherlands) [32]. Quadriceps muscle function (peak strength and endurance) was evaluated with a Biodex system 3 (Biodex Medical Systems Inc., New York, US). Patients performed thirty volitional maximal knee extensions at an angular velocity of $90^{\circ}$ per second, while seated upright and with the hip joint in $90^{\circ}$ of flexion [33].

\section{Experimental setup}

Before and after PR, patients were instructed to walk on a split-belt instrumented treadmill within a virtual reality environment of a hallway (Gait Real-time Analysis Interactive Lab, GRAIL, Motek Medical B.V., the Netherlands). A Vicon motion tracking system with 10 Bonita cameras (Vicon Nexus, Oxford, UK) was used to track markers at $100 \mathrm{~Hz}$ [4]. These markers were placed on anatomical landmarks of the patient, according to the Human Body Model of the lower-limb (HBM1) [34]. Force plate data were sampled at $1000 \mathrm{~Hz}$ in synchronization with the motion capture system. Self-paced treadmill walking was enabled via a built-in controller algorithm that regulates the belt's speed, as described in [4]. Patients performed one familiarization session. Then, two GRAIL-based 6MWTs were performed between pre-rehabilitation assessment and the first week of PR. Perceived dyspnea and fatigue (Borg scale), heart rate and pulse oxygen saturation levels were measured prior and post each 6MWT [27]. Patients performed two additional GRAIL-based 6MWTs between the last week of PR and the outcome assessment.

\section{Data analyses}

The GRAIL-based 6MWT with the longest walk distance for each subject in pre and post PR was used for analyses. The first 60 seconds (to minimize start-up effects) and the last 15 seconds (to minimize deceleration effects of the treadmill) were excluded. Marker and 
force plate date were processed in custom MATLAB software (Mathworks Inc., Natick, USA), as previously described [4].

All data-analysis of gait was performed on 444 consecutive steps per trial, based on the lowest number of steps of all GRAIL-based 6MWTs (ranged 444-684). Gait characteristics can be evaluated by computing spatiotemporal gait parameters (stride time, stride length and step width) and movement patterns of the center of mass of the subject's body. Stride time was calculated as the time from one heel contact to the next ipsilateral heel contact. Stride length was defined as the distance between the toe marker and the ipsilateral toe marker at each heel contact in the anteroposterior direction, accounted for treadmill speed. Step width was defined as the distance between the toe markers in mediolateral direction between both feet at heel strike. The center of mass velocity $\left(\mathrm{CoM}_{\mathrm{vel}}\right)$ was computed using the position of the four pelvis markers.

The amount of stride-to-stride fluctuations in gait parameters was quantified by the standard deviation and coefficient of variation of spatiotemporal gait parameters ( CoV = [standard deviation/mean]). Increased standard deviation and CoV values reflect a disturbed gait, as has been found in aging and disease [15].

The patterns within stride-to-stride fluctuations were assessed using various measures, including sample entropy and local divergence exponent (See Supplementary Material 6.A for further description of the calculation of these measures) [8, 35, 36]. In short, sample entropy is a measure of predictability in the time series [36]. Sample entropy was calculated using $m=2, r=0.2$ and $n=444$. Reduced sample entropy values reflect a more predictable gait pattern, which has been associated with a less healthy locomotor system [37]. Stride time sample entropy seemed to be a product of the parameter selection, therefore only stride length and step width sample entropy were reported.

The $\mathrm{CoM}_{\mathrm{vel}}$ was subjected to consistency analysis, in which the local divergence exponent is calculated. The method to compute this has been described elsewhere [35]. Increased local divergent exponents reflected less consistent organization of movement patterns of the patient during walking, which has been found in falls and disease [9, 10, 35]. Briefly, the center of mass velocity time series was reconstructed into a multidimensional space and the distances between these points were calculated as a function of time and averaged over all data points to obtain the average logarithmic rate of divergence. The slope of the divergence curve provided an estimate of the local divergence exponent, which quantifies the separation of the center of mass trajectories over time. 


\section{Statistics}

Data are presented as mean difference ( $95 \%$ Confidence Interval) unless otherwise stated. Differences in the 6MWT outcome parameters and gait characteristics between pre and post PR were compared using either paired sample T-tests or nonparametric Wilcoxon signed-rank tests. A sub-analysis was performed on good and poor responders. The minimal important difference of $30 \mathrm{~m}$ has been used to differentiate poor responders $(<30$ $\mathrm{m}$ improvement) and good responders ( $\geq 30 \mathrm{~m}$ improvement) to PR in the GRAIL-based 6MWT [26, 38]. Either independent sample T-tests or nonparametric Mann-Whitney U tests were conducted to assess the differences between poor responders and good responders at baseline and the amount of changes after PR. All statistical analyses were performed in SPSS Statistics software 22.0 (IBM, New York, USA). The level of significance for all analyses was set at $p \leq 0.01$.

\section{Results}

\section{Baseline subject characteristics}

Patients had mild to severe COPD (Table 6.1). Mean body mass index (BMI) was 26.9 $\pm 5.2 \mathrm{~kg} / \mathrm{m}^{2}$. Patients performed the TUG test in $8.8 \pm 1.3$ seconds (Table 6.2). Mean quadriceps muscle strength was $71 \pm 14 \%$ predicted. Patients walked $512 \pm 67 \mathrm{~m}$ during the best overground $6 \mathrm{MWT}$, corresponding to $80 \pm 10 \%$ predicted, and $506 \pm 75 \mathrm{~m}$ during the best pre-rehabilitation GRAIL-based 6MWT (Table 6.3). Most patients achieved their largest distance during the second 6MWT in both overground and GRAIL setting, 68\% and $66 \%$, respectively.

\section{Changes after pulmonary rehabilitation}

Fat-free mass and fat-free mass index, quadriceps muscle strength and endurance, and overground 6MWD increased significantly after PR (Table 6.2). The GRAIL-based 6MWD increased with $30 \mathrm{~m}$ after PR (95\% Cl: 14-46 m, $p<0.001$; Table 6.3). Mean stride time reduced after PR (MD: -0.02 s, $95 \% \mathrm{Cl}:-0.03-0.01 \mathrm{~s}, p=0.001)$. Patients with COPD did not demonstrate significant changes in the standard deviation, CoV or predictability of spatiotemporal gait parameters, neither a change in consistency in the organization of center of mass patterns was found after PR. 
Table 6.1. Patient demographic characteristics at baseline

\begin{tabular}{|c|c|c|}
\hline Parameter & & $\begin{array}{l}\text { Pre-pulmonary rehabilitation } \\
n=44\end{array}$ \\
\hline Age, years & & $62.2(7.5)$ \\
\hline Gender M/F, $n$ & & $25 / 19$ \\
\hline Weight, kg & & $76.5(16.6)$ \\
\hline Height, m & & $1.68(0.18)$ \\
\hline $\mathrm{BMI}, \mathrm{kg} / \mathrm{m}^{2}$ & & $26.9(5.2)$ \\
\hline $\mathrm{FEV}_{1} / \mathrm{FVC}$ & & $0.42(0.12)$ \\
\hline $\mathrm{FEV}_{1} \%$ predicted & & $55.88(19.73)$ \\
\hline \multirow[t]{4}{*}{ GOLD group, $n$} & I & $6(13.6 \%)$ \\
\hline & II & $18(40.9 \%)$ \\
\hline & III & $17(38.6 \%)$ \\
\hline & IV & $3(6.8 \%)$ \\
\hline Never smoker, $n$ & & $1(2.3 \%)$ \\
\hline Former smoker, $n$ & & 41 (93.2\%) \\
\hline Current smoker, $n$ & & $2(4.6 \%)$ \\
\hline Pack years & & $41.55(20.6)$ \\
\hline
\end{tabular}

Note: Data are expressed as mean (SD) or number (\%). Abbreviations: BMI, body mass index; $\mathrm{FEV}_{1}$, forced expiratory volume in $1 \mathrm{~s}$; FVC, forced vital capacity; GOLD, Global Initiative for Chronic Obstructive Lung Disease.

\section{Good versus poor responders}

Twenty-four patients (55\%) achieved an improvement of 30 meters or more on the GRAILbased 6MWT, which qualified them as good responders. At baseline, no differences in 6MWT outcomes and gait characteristics were found between good and poor responders, except for mean step width, which was significantly lower in the good responders at baseline (Table 6.3 and Table 6.4). The good responders had a significantly greater improvement in 6MWD, both overground and using the GRAIL, compared to the poor responders (Figure 6.1). The good responders had a significantly greater increase in stride length and a greater decrease in stride time as compared to the poor responders (MD: 0.14 m, 95\% Cl: 0.09 - 0.19 m, $p<0.001$; MD: -0.05 s, 95\% Cl: $-0.07--0.03, p<0.001$, respectively). No differences were found in the change in $\mathrm{CoV}$ of spatiotemporal gait parameters between the groups after PR. The change in predictability in spatiotemporal gait parameters and the change in local dynamic stability of the $\mathrm{CoM}_{\text {vel }}$ did not reach a statistically significant difference after PR. 
Table 6.2. Clinical outcomes before and after pulmonary rehabilitation

\begin{tabular}{|c|c|c|c|c|c|c|}
\hline \multirow[b]{2}{*}{ Test } & \multirow[b]{2}{*}{ Parameter } & \multicolumn{2}{|c|}{$\begin{array}{l}\text { Pre pulmonary } \\
\text { rehabilitation }\end{array}$} & \multicolumn{2}{|c|}{$\begin{array}{l}\text { Post pulmonary } \\
\text { rehabilitation }\end{array}$} & \multirow[b]{2}{*}{$p$-value } \\
\hline & & $n$ & Value & $n$ & value & \\
\hline Body & Total FM, kg & 43 & $27.3(9.2)$ & 43 & $27.1(8.6)$ & 0.745 \\
\hline \multirow[t]{2}{*}{ composition } & Total FFM, kg & 43 & $47.8(9.5)^{*}$ & 43 & $48.7(9.7)$ & $<0.001^{\dagger}$ \\
\hline & FFMI, $\mathrm{kg} / \mathrm{m}^{2}$ & 43 & $16.8(2.4)$ & 43 & $17.1(2.4)$ & $<0.001$ \\
\hline Quadriceps & Peak torque, N/m & 38 & $106(33)$ & 38 & $114(35)$ & $<0.001$ \\
\hline \multirow{3}{*}{ muscle function } & Peak torque, \% predicted & 38 & $71(14)$ & 38 & $77(14)$ & $<0.001$ \\
\hline & Total work, J & 38 & $1978(625)$ & 38 & $2220(669)^{*}$ & $<0.001^{\dagger}$ \\
\hline & Work fatigue, $\%$ & 38 & $43(11)$ & 38 & $39(9)^{*}$ & $0.048^{\dagger}$ \\
\hline TUG test & Time, $\mathrm{s}$ & 44 & $8.8(1.3)$ & 44 & $8.7(1.1)$ & 0.653 \\
\hline Overground & 6MWD, m & 44 & $512(67)$ & 44 & $535(71)$ & 0.003 \\
\hline \multirow[t]{9}{*}{$6 \mathrm{MWT}$} & Walking speed, $\mathrm{m} / \mathrm{s}$ & 44 & $1.42(0.18)$ & 44 & $1.5(0.2)$ & 0.003 \\
\hline & $\mathrm{SpO}_{2}$ rest, \% & 44 & $94(2)$ & 44 & $95(2)^{*}$ & $0.760^{\dagger}$ \\
\hline & $\mathrm{SpO}_{2}$ end, $\%$ & 44 & $88(6)^{*}$ & 44 & $87(6)$ & $0.055^{\dagger}$ \\
\hline & HR rest, bpm & 44 & 85 (14) & 44 & 80 (14) & 0.014 \\
\hline & HR end, bpm & 44 & $115(17)$ & 44 & 117 (17) & 0.318 \\
\hline & Dyspnea rest, score & 44 & $1.2(1.1)^{*}$ & 44 & $1.0(1.0)$ & $0.243^{\dagger}$ \\
\hline & Dyspnea end, score & 44 & $5.6(2.0)^{*}$ & 44 & $5.1(2.2)$ & $0.108^{\dagger}$ \\
\hline & Fatigue rest, score & 44 & $1.3(1.5)^{*}$ & 44 & $1.1(1.2)^{*}$ & $0.172^{\dagger}$ \\
\hline & Fatigue end, score & 44 & $5.0(2.5)$ & 44 & $4.3(2.4)$ & 0.030 \\
\hline
\end{tabular}

Note: Data are expressed as mean (SD). Abbreviations: FM, fat mass; FFM, fat free mass; FFMI, fat free mass index; TUG, Timed Up and Go; 6MWT, 6-minute walk test; 6MWD, 6-minute walk distance; $\mathrm{SpO}_{2}$, pulse oxygen saturation level; HR, heart rate; bpm, beats per minute.

*: Non-parametric value.

†: Non-parametric Wilcoxon signed-rank test. 
Table 6.3. The 6MWT outcomes and gait parameters during the GRAIL-based 6MWT before and after pulmonary rehabilitation

\begin{tabular}{|c|c|c|c|c|c|c|}
\hline \multirow[b]{2}{*}{ Test } & \multirow[b]{2}{*}{ Parameter } & \multicolumn{2}{|c|}{$\begin{array}{l}\text { Pre pulmonary } \\
\text { rehabilitation }\end{array}$} & \multicolumn{2}{|c|}{$\begin{array}{l}\text { Post pulmonary } \\
\text { rehabilitation }\end{array}$} & \multirow[b]{2}{*}{$p$-value } \\
\hline & & $n$ & value & $n$ & value & \\
\hline \multirow[t]{10}{*}{ 6MWT } & 6MWD, m & 44 & $506(75)$ & 44 & $537(82)$ & $<0.001$ \\
\hline & Walking speed, $\mathrm{m} / \mathrm{s}$ & 44 & $1.43(0.19)$ & 44 & $1.48(0.23)$ & 0.056 \\
\hline & $\mathrm{SpO}_{2}$ rest, $\%$ & 44 & $96(2)$ & 44 & $95(2)$ & 0.357 \\
\hline & $\mathrm{SpO}_{2}$ end, $\%$ & 44 & $92(5)^{\#}$ & 44 & $93(4)^{*}$ & $0.773^{\dagger}$ \\
\hline & HR rest, bpm & 44 & $82(16)$ & 44 & $80(15)$ & 0.252 \\
\hline & HR end, bpm & 44 & $104(20)$ & 44 & $103(21)$ & 0.599 \\
\hline & Dyspnea rest, score & 44 & $1.2(1.1)^{*}$ & 44 & $1.0(0.9)^{*}$ & $0.120^{\dagger}$ \\
\hline & Dyspnea end, score & 44 & $5.3(2.2)^{*}$ & 44 & $4.6(2.2)^{*}$ & $0.019^{\dagger}$ \\
\hline & Fatigue rest, score & 44 & $1.2(1.2)^{*}$ & 44 & $1.1(1.1)^{*}$ & $0.487^{\dagger}$ \\
\hline & Fatigue end, score & 44 & $4.6(2.3)$ & 44 & $4.4(2.4)$ & 0.494 \\
\hline \multirow[t]{14}{*}{ Gait } & Mean stride time, $\mathrm{s}$ & 44 & $1.02(0.08)$ & 44 & $1.00(0.08)$ & 0.001 \\
\hline & Mean stride length, $\mathrm{m}$ & 44 & $1.45(0.19)$ & 44 & $1.48(0.18)^{*}$ & $0.037^{\dagger}$ \\
\hline & Mean step width, m & 44 & $0.18(0.05)$ & 44 & $0.18(0.05)$ & $0.101^{\dagger}$ \\
\hline & SD stride time, $\mathrm{s}$ & 44 & $0.02(0.01)$ & 44 & $0.02(0.01)$ & $0.599^{\dagger}$ \\
\hline & SD stride length, $\mathrm{m}$ & 44 & $0.05(0.02)$ & 44 & $0.04(0.03)$ & $0.036^{\dagger}$ \\
\hline & SD step width, m & 44 & $0.02(0.01)$ & 44 & $0.02(0.001)$ & $0.916^{\dagger}$ \\
\hline & CoV stride time & 44 & $0.02(0.01)^{*}$ & 44 & $0.02(0.01)^{*}$ & $0.889^{\dagger}$ \\
\hline & CoV stride length & 44 & $0.03(0.02)^{*}$ & 44 & $0.03(0.03)^{*}$ & $0.024^{\dagger}$ \\
\hline & CoV step width & 44 & $0.15(0.07)^{*}$ & 44 & $0.15(0.05)^{*}$ & $0.363^{\dagger}$ \\
\hline & SE stride length & 44 & $1.17(0.17)^{*}$ & 44 & $1.21(0.17)^{*}$ & $0.037^{\dagger}$ \\
\hline & SE step width & 44 & $1.43(0.04)^{*}$ & 44 & $1.43(0.05)^{*}$ & $0.825^{\dagger}$ \\
\hline & LDE CoMvel-ML & 44 & $2.83(0.17)$ & 44 & $2.77(0.19)^{*}$ & $0.031^{\dagger}$ \\
\hline & LDE CoMvel-V & 44 & $2.78(0.14)$ & 44 & $2.79(0.14)$ & 0.828 \\
\hline & LDE CoMvel-AP & 44 & $2.75(0.15)^{*}$ & 44 & $2.70(0.15)$ & $0.018^{\dagger}$ \\
\hline
\end{tabular}

Note: Data are expressed as mean (SD). Abbreviations: 6MWT, 6-minute walk test; 6MWD, 6-minute walk distance; $\mathrm{SpO}_{2}$, pulse oxygen saturation level; bmp, beats per minute; $\mathrm{SD}$, standard deviation; CoV, coefficient of variation; SE, sample entropy; LDE, local divergence exponent; CoMvel, center of mass velocity; ML, mediolateral direction; $\mathrm{V}$, vertical direction; $\mathrm{AP}$, anteroposterior direction.

*: Non-parametric value.

t: Non-parametric Wilcoxon signed-rank test. 


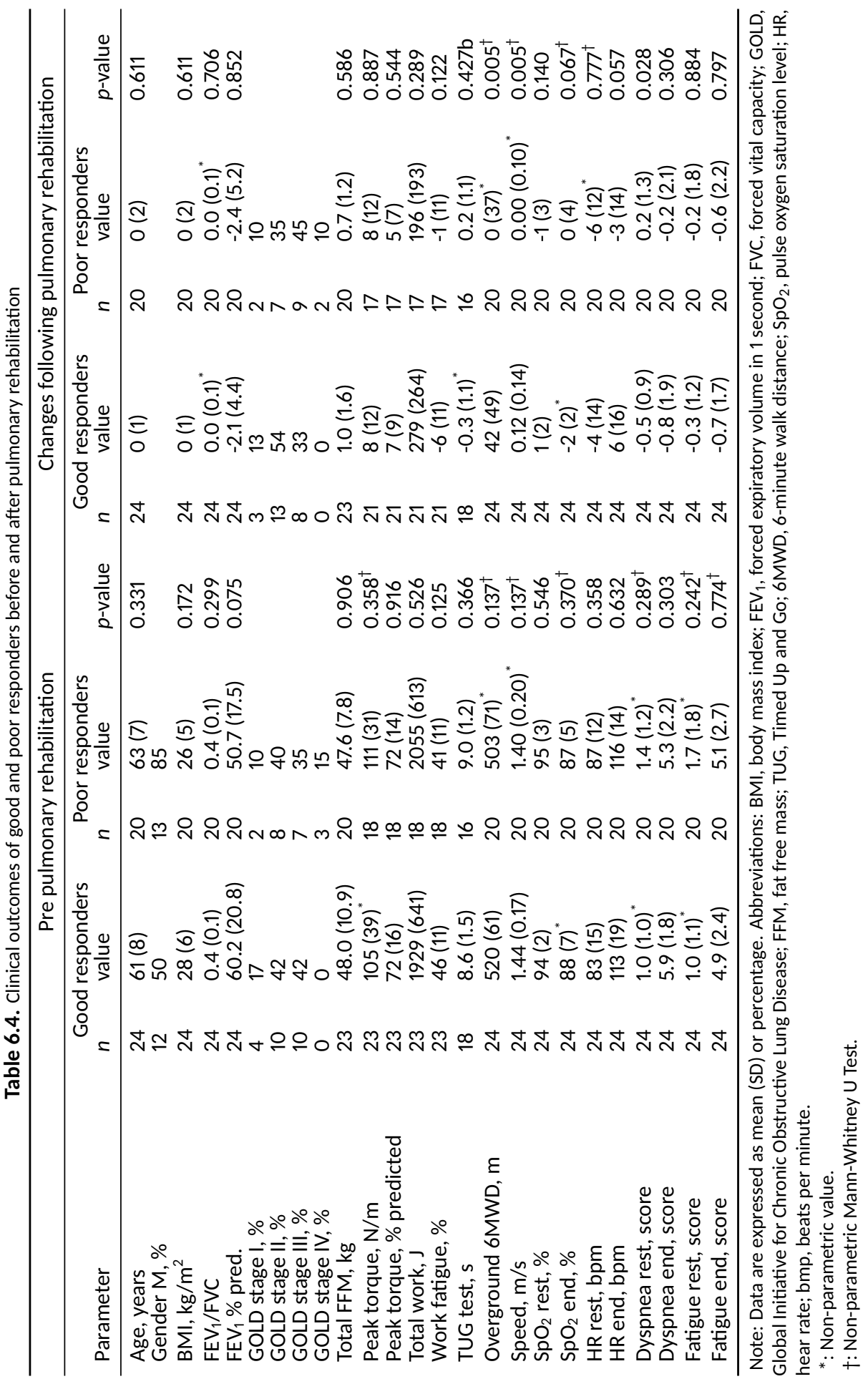




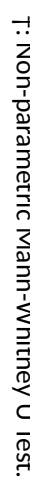

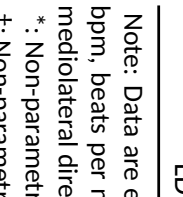

$\stackrel{\text { D. }}{\stackrel{7}{7}}$

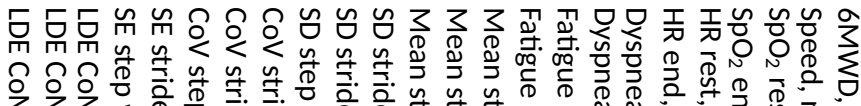

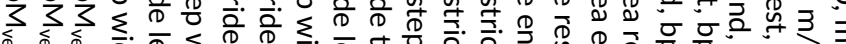

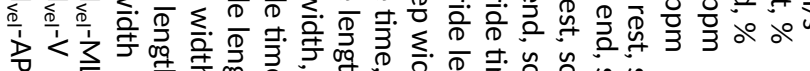

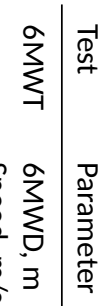

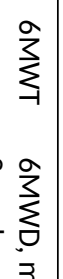

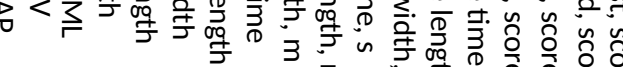

을

䙳.

3

3

$\frac{0}{\bar{D}} \frac{\mathrm{O}}{\mathrm{D}}$

实高

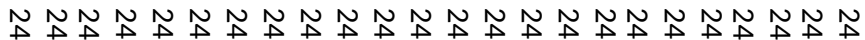

施.

응

过雼: 学

옹용

윽 옹.

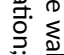

留

气ै

夋 ?

$\frac{\overline{0}}{\overline{0}} \bar{z}$

일

शे

$\checkmark$ o

O응 OO OO

它立它

N

$\dot{\vec{v}}$ 으

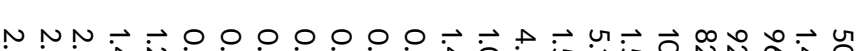

म

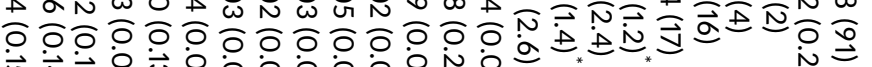

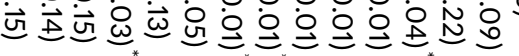

i

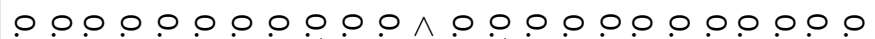

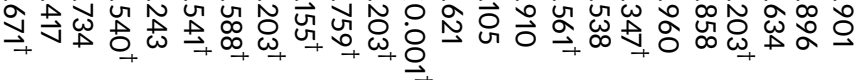

它

잉

$\stackrel{2}{2}$

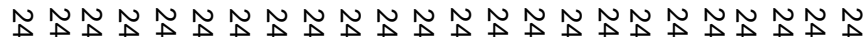

ํ.?ㄹ.

召

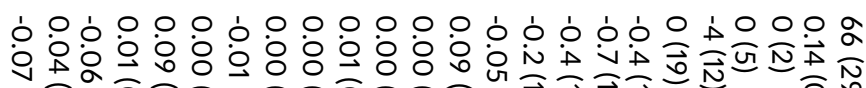

员 븡

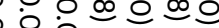

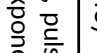

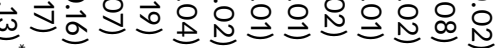

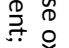

ᄋิ oั

空

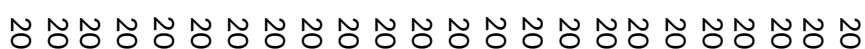

$\overrightarrow{\overrightarrow{0}}$

2

กิ

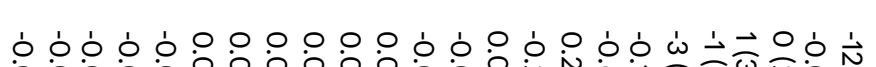

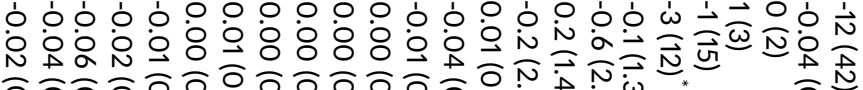

它

उ市

$\omega$

品

要

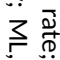

iv

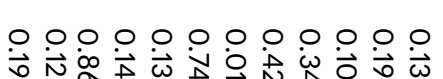

$\wedge \wedge .0 .000 .0 \wedge \wedge$

ज़

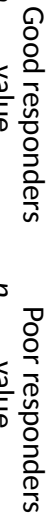




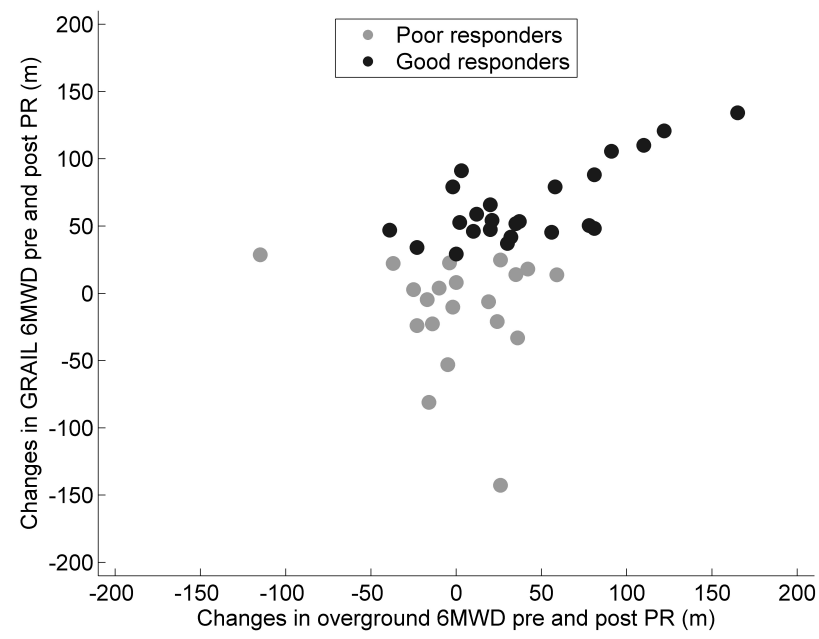

Figure 6.1. Changes in 6MWD in good and poor responders following pulmonary rehabilitation (PR) in the overground and GRAIL condition. Note: The good responders are depicted in black dots and the poor responders in grey dots.

\section{Discussion}

This study aimed to evaluate whether, and to what extent, a comprehensive PR program affects gait characteristics in patients with COPD during the GRAIL-based 6MWT. Patients with COPD demonstrated shorter mean stride times during the post PR GRAILbased 6MWT. No changes in the amount of, and patterns within stride-to-stride fluctuations were found in patients following PR. In addition, good responders to PR showed improvements in mean stride time and stride length as compared to poor responders. No differences in stride-to-stride fluctuations were found between these groups after PR. The present findings suggest that a comprehensive PR program does improve walking speed in patients with COPD, whereas stride-to-stride fluctuations did not change. Research on gait impairments in COPD and identifying training modalities that improve gait function in COPD are therefore recommended.

Gait assessment may be of clinical value as gait abnormalities have been reported in patients with COPD, including reduced cadence, reduced stride length and increased double support time as compared to healthy subjects [3-7]. Though PR has proven to increase gait speed in patients with COPD [39], a comprehensive analysis of gait characteristics after PR is lacking. In the current study, patients with COPD improved their walk distance during the GRAIL-based 6MWT after PR, which also reached a clinical significant 
improvement [26]. This improvement in walking distance was achieved by a reduced mean stride time, and thus faster walking speeds. Moreover, patients with COPD tended to take longer strides contributing to an increased 6MWD. However, stride length changes were not found to be statistically significant.

Stride-to-stride fluctuations in gait may represent the capability of the locomotor system to make flexible adaptations during walking [14]. Healthy subjects are characterized by a healthy amount of, and patterns within stride-to-stride fluctuations [40]. These strideto-stride fluctuations are associated with fall risk and may deteriorate with aging and disease [4, 6, 9, 10, 12, 41, 42]. In community dwelling older adults, improvements in stride-to-stride fluctuations can be achieved following physical training [43, 44]. Exercise training is a key element in PR [23] and demonstrated to improve skeletal muscle function $[22,45,46]$. As skeletal muscles are important components of the locomotor system for walking, PR was hypothesized to have beneficial effects on the amount of fluctuations, predictability and consistency of movement patterns in patients with COPD. In the current study, patients with COPD did not demonstrate less variable gait characteristics, less predictable patterns of gait characteristics and increased consistency of the center of mass movement patterns. Patients did show increased fat-free mass (e.g. muscle mass), and lower-limb muscle strength and endurance. The combination of individualized strength, interval and endurance training resulted in improvements in lower-limb muscle function. However, these improvements do not necessarily translate into improvements in strideto-stride fluctuations in patients with COPD.

Interventions to improve walking have traditionally been multifactorial (i.e. strengthening, endurance and flexibility programs). These interventions focus on improving the physiologic capacity in body systems (i.e. musculoskeletal and cardiopulmonary systems) that contribute to walking, but do not include task specific exercises necessary to make use of the physiological capacity in body systems for walking (e.g. integrate movement and posture to improve efficiency of the physiological capacity) [47]. Therefore, taskoriented exercise training of walking could be beneficial to improve gait function, and more specifically in stride-to-stride fluctuations in COPD.

Response to PR differs in patients with COPD [25]. The current study showed that 55\% of the patients were good responders in terms of improved functional exercise capacity during the GRAIL-based 6MWT ( $\geq 30 \mathrm{~m}$ ), with an average increase in the 6MWD of $66 \mathrm{~m}$ after PR. The poor responders showed a decrease of $12 \mathrm{~m}$ during the GRAIL-based 6MWT after PR. The difference in step width at baseline was minimal between the groups and after PR this was minimal between the groups. This difference was not considered as clinically relevant. The good responders were therefore considered similar to the poor responders in terms of mean gait characteristics. Larger increase in mean walking speed 
for the good responders was accompanied by a shorter mean stride time and longer mean stride lengths.

In contrast to mean spatiotemporal gait parameters between the good and poor responders, the change in the amount of, and patterns within, stride-to-stride fluctuations did not reach significance. This could partially explained by that exercise training in PR was not specifically targeted to systems that contribute to balance and gait control. Other parameters may have contributed to the faster walking speed in the good responders. The good responders tended to start with lower dyspnea and fatigue levels compared to the poor responders during the post PR GRAIL-based 6MWT. Interestingly, some poor responders showed improvements in lower-limb muscle function. This indicates that other factors may be associated with decreased walking speed, such as higher fatigue levels prior to the post PR GRAIL-based 6MWT. Furthermore, differences in ventilator reserve may be important. However, this was not assessed in the present study.

The present study has some limitations. Externally-paced treadmill walking affects gait parameters and its stride-to-stride fluctuations. The GRAIL system, however, enables selfpaced treadmill walking, using a feedback-controlled algorithm to adapt treadmill speed to the user while walking. The GRAIL has shown to increase walking speed variability [48], thereby suggesting that self-paced treadmill walking allows for a more natural control of walking speed [49]. Patients with COPD who were most severely limited in their mobility and therefore using a walking aid were not eligible for the GRAIL test. It would be interesting to investigate whether alterations in gait characteristics are present for patients with more severe COPD, especially given the trend that lower-limb muscle weakness is more prevalent with more severe COPD [50]. Another limitation is that the assessment of lower-limb muscle strength and endurance in our study only targeted the quadriceps muscles. Reduced muscle strength has also been observed in the distal muscles of the lower-limbs [51]. Future studies should include the assessment of distal lower-limbs to gain a better understanding of distal lower-limb muscle dysfunction and gait characteristics in patients with COPD.

It was postulated that exercise training, and in particular changes in lower-limb muscle function, could have positive effects on gait characteristics in COPD. This was partially confirmed by the findings of the present study, as walking speed increased. However, stride-to-stride fluctuations did not improve following PR. The current PR program was not specifically aimed to improve gait function and balance in patients with COPD. Moreover, in contrast to the study by Wang et al. [44], our study did not incorporate balance training specifically in PR. Consequently, this might explain the lack of changes in strideto-stride fluctuations. It may be important to present exercises targeting to various systems for balance control and in different situations. We therefore propose to perform 
future research on gait and balance training in PR for patients with COPD. To date, different modalities of gait training are available, including perturbation training and dual task training $[52,53]$, that could be used to assess the effect of gait and/or balance training on gait characteristics in COPD. In particular, improvements in stride-to-stride fluctuations will be important, as these measures are associated with increased fall risk in older adults and the high incidence of fall risk present in COPD [11].

\section{Conclusions}

This study showed that patients with COPD demonstrate shorter stride times during the GRAIL-based 6MWT after a comprehensive PR program. Though improvements in exercise capacity, body composition and quadriceps strength were found in patients with COPD following PR, stride-to-stride fluctuations did not improve. In addition, differences in mean gait characteristics were found between good and poor responders, while strideto-stride fluctuations were not discriminative. These findings indicate that the current PR does not alter stride-to-stride fluctuations in patients with COPD. Additional training programs specifically targeting balance and gait function may be beneficial in improving gait characteristics in patients with COPD.

\section{Acknowledgments}

The authors thank S.M. Bruijn and J.M. Yentes for assistance with data-analysis. We are grateful to the study participants and the staff of CIRO. 


\section{Bibliography}

1. Annegarn, J. et al. Problematic activities of daily life are weakly associated with clinical characteristics in COPD. J Am Med Dir Assoc 13, 284-90 (2012).

2. Nakken, N. et al. Patient versus proxy-reported problematic activities of daily life in patients with COPD. Respirology 22, 307-314 (2017).

3. Annegarn, J. et al. Differences in walking pattern during 6-min walk test between patients with COPD and healthy subjects. PLoS One 7, e37329 (2012).

4. Liu, W. Y. et al. Spatiotemporal gait characteristics in patients with COPD during the Gait Real-time Analysis Interactive Lab-based 6-minute walk test. PLoS One 12, e0190099 (2017).

5. Nantsupawat, N. et al. Gait Characteristics in Patients With Chronic Obstructive Pulmonary Disease. J Prim Care Community Health 6, 222-6 (2015).

6. Yentes, J. M. et al. Patients with Chronic Obstructive Pulmonary Disease Walk with Altered Step Time and Step Width Variability as Compared with Healthy Control Subjects. Ann Am Thorac Soc 14, 858-866 (2017).

7. Zago, M. et al. Gait analysis in patients with chronic obstructive pulmonary disease: a systematic review. Gait Posture 61, 408-415 (2018).

8. Hausdorff, J. M. et al. Altered fractal dynamics of gait: reduced stride-interval correlations with aging and Huntington's disease. J Appl Physiol (1985) 82, 262-9 (1997).

9. Myers, S. A. et al. Gait variability is altered in patients with peripheral arterial disease. J Vasc Surg 49, 924-931 e1 (2009).

10. Buzzi, U. H. et al. Nonlinear dynamics indicates aging affects variability during gait. Clin Biomech (Bristol, Avon) 18, 435-43 (2003).

11. Hausdorff, J. M., Rios, D. A. \& Edelberg, H. K. Gait variability and fall risk in community-living older adults: a 1-year prospective study. Arch Phys Med Rehabil 82, 1050-6 (2001).

12. Maki, B. E. Gait changes in older adults: predictors of falls or indicators of fear. J Am Geriatr Soc 45, 313-20 (1997).

13. Moe-Nilssen, R. \& Helbostad, J. L. Interstride trunk acceleration variability but not step width variability can differentiate between fit and frail older adults. Gait \& Posture 21, 164-70 (2005).

14. Stergiou, N., Harbourne, R. \& Cavanaugh, J. Optimal movement variability: a new theoretical perspective for neurologic physical therapy. J Neurol Phys Ther 30, 120-9 (2006).

15. Hausdorff, J. M. et al. Gait variability and basal ganglia disorders: stride-to-stride variations of gait cycle timing in Parkinson's disease and Huntington's disease. Mov Disord 13, 428-37 (1998).

16. Roig, M. et al. Falls in patients with chronic obstructive pulmonary disease: a call for further research. Respir Med 103, 1257-69 (2009).

17. Marques, N. R. et al. Association between energy cost of walking, muscle activation, and biomechanical parameters in older female fallers and non-fallers. Clin Biomech (Bristol, Avon) 28, 330-6 (2013).

18. Shin, S. et al. Lower extremity muscle quality and gait variability in older adults. Age Ageing 41, 595-9 (2012).

19. Lord, S. R. et al. The effect of exercise on gait patterns in older women: a randomized controlled trial. J Gerontol A Biol Sci Med Sci 51, M64-70 (1996).

20. Mathur, S., Brooks, D. \& Carvalho, C. R. Structural alterations of skeletal muscle in COPD. Front Physiol 5, 104 (2014).

21. De Brandt, J. et al. Changes in structural and metabolic muscle characteristics following exercise-based interventions in patients with COPD: a systematic review. Expert Rev Respir Med 10, 521-45 (2016).

22. De Brandt, J. et al. Changes in lower limb muscle function and muscle mass following exercise-based interventions in patients with chronic obstructive pulmonary disease: A review of the English-language literature. Chron Respir Dis 15, 182-219 (2018). 
23. Spruit, M. A. et al. An official American Thoracic Society/European Respiratory Society statement: key concepts and advances in pulmonary rehabilitation. Am J Respir Crit Care Med 188, e13-64 (2013).

24. Augustin, I. M. L. et al. Comprehensive Lung Function Assessment Does not Allow to Infer Response to Pulmonary Rehabilitation in Patients with COPD. J Clin Med 8 (2018).

25. Spruit, M. A. et al. Differential response to pulmonary rehabilitation in COPD: multidimensional profiling. Eur Respir J 46, 1625-35 (2015).

26. Holland, A. E. et al. An official European Respiratory Society/American Thoracic Society technical standard: field walking tests in chronic respiratory disease. Eur Respir J 44, 1428-46 (2014).

27. Liu, W. Y. et al. Reproducibility and Validity of the 6-Minute Walk Test Using the Gait Real-Time Analysis Interactive Lab in Patients with COPD and Healthy Elderly. PLoS One 11, e0162444 (2016).

28. Vogelmeier, C. F. et al. Global Strategy for the Diagnosis, Management, and Prevention of Chronic Obstructive Lung Disease 2017 Report. GOLD Executive Summary. Am J Respir Crit Care Med 195, 557-582 (2017).

29. Spruit, M. A. et al. Integration of pulmonary rehabilitation in COPD. Lancet 371, 12-3 (2008).

30. Garber, C. E. et al. American College of Sports Medicine position stand. Quantity and quality of exercise for developing and maintaining cardiorespiratory, musculoskeletal, and neuromotor fitness in apparently healthy adults: guidance for prescribing exercise. Med Sci Sports Exerc 43, 1334-59 (2011).

31. Podsiadlo, D. \& Richardson, S. The timed Up \& Go: a test of basic functional mobility for frail elderly persons. J Am Geriatr Soc 39, 142-8 (1991).

32. Van de Bool, C. et al. Quality of dietary intake in relation to body composition in patients with chronic obstructive pulmonary disease eligible for pulmonary rehabilitation. Eur J Clin Nutr 68, 159-65 (2014).

33. Mathur, S., Makrides, L. \& Hernandez, P. Test-retest reliability of isometric and isokinetic torque in patients with chronic obstructive pulmonary disease. Physiother Can. 56, 94-101 (2004).

34. Van den Bogert, A. J. et al. A real-time system for biomechanical analysis of human movement and muscle function. Med Biol Eng Comput 51, 1069-77 (2013).

35. Bruijn, S. M. et al. Assessing the stability of human locomotion: a review of current measures. J R Soc Interface 10, 20120999 (2013).

36. Yentes, J. M. et al. The appropriate use of approximate entropy and sample entropy with short data sets. Ann Biomed Eng 41, 349-65 (2013).

37. Jin, Y. et al. Entropy change of biological dynamics in COPD. Int J Chron Obstruct Pulmon Dis 12, 29973005 (2017).

38. Polkey, M. I. et al. Six-minute-walk test in chronic obstructive pulmonary disease: minimal clinically important difference for death or hospitalization. Am J Respir Crit Care Med 187, 382-6 (2013).

39. McClellan, R. et al. Pulmonary Rehabilitation Increases Gait Speed in Patients With Chronic Lung Diseases. Health Serv Res Manag Epidemiol 1, 2333392814533659 (2014).

40. Stergiou, N. \& Decker, L. M. Human movement variability, nonlinear dynamics, and pathology: is there a connection? Hum Mov Sci 30, 869-88 (2011).

41. Dingwell, J. B. \& Cusumano, J. P. Nonlinear time series analysis of normal and pathological human walking. Chaos 10, 848-863 (2000).

42. Lipsitz, L. A. \& Goldberger, A. L. Loss of 'complexity' and aging. Potential applications of fractals and chaos theory to senescence. JAMA 267, 1806-9 (1992).

43. Beauchet, O. et al. Physical training-related changes in gait variability while single and dual tasking in older adults: magnitude of gait variability at baseline matters. Eur J Phys Rehabil Med 49, 857-64 (2013)

44. Wang, R. Y. et al. Effects of combined exercise on gait variability in community-dwelling older adults. Age (Dordr) 37, 9780 (2015).

45. Mador, M. J. et al. Endurance and strength training in patients with COPD. Chest 125, 2036-45 (2004).

46. Nici, L. et al. American Thoracic Society/European Respiratory Society statement on pulmonary rehabilitation. Am J Respir Crit Care Med 173, 1390-413 (2006). 
47. Brach, J. S. \& Vanswearingen, J. M. Interventions to Improve Walking in Older Adults. Curr Transl Geriatr Exp Gerontol Rep 2 (2013).

48. Sloot, L. H., van der Krogt, M. M. \& Harlaar, J. Self-paced versus fixed speed treadmill walking. Gait Posture 39, 478-84 (2014).

49. Plotnik, M. et al. Self-selected gait speed-over ground versus self-paced treadmill walking, a solution for a paradox. J Neuroeng Rehabil 12, 20 (2015).

50. Seymour, J. M. et al. The prevalence of quadriceps weakness in COPD and the relationship with disease severity. Eur Respir J 36, 81-8 (2010).

51. Gagnon, P. et al. Distal leg muscle function in patients with COPD. COPD 10, 235-42 (2013).

52. McCrum, C. et al. A systematic review of gait perturbation paradigms for improving reactive stepping responses and falls risk among healthy older adults. Eur Rev Aging Phys Act 14, 3 (2017).

53. Mirelman, A. et al. V-TIME: a treadmill training program augmented by virtual reality to decrease fall risk in older adults: study design of a randomized controlled trial. BMC Neurol 13, 15 (2013). 



\section{Supplementary Material}

\section{Measures to quantify the patterns within stride-to-stride fluctuations}

Sample entropy quantifies predictability and describes the predictability of gait cycles. The method to compute this has been described previously [1]. A perfectly repeatable time series would reflect a sample entropy value of approximately 0 , while a completely random time series would reflect a sample entropy value extending towards infinity. The relative consistency of the group averages was examined for several combinations of input parameters of the tolerance radius $r$ and vector length $m$ [1]. The $r$ was chosen as $0.2^{*}$ standard deviation of the time series and $m$ was chosen as 2 for this study (Figure 6.A.1). In contrast to stride length and step width time series, stride time sample entropy seems to be a product of the parameter selection. Sample entropy values tended to direct to predictability in stride time with increasing $r$. Predictability of stride time itself could not be determined. Stride time sample entropy was therefore not included for statistical analysis.

Consistency in the organization of movement patterns was calculated by computing the local divergence exponent, which quantifies the exponential rate of divergence of adjacent trajectories in state space and has been described elsewhere [2]. A lower local divergence exponent reflects a more consistent organization of movement patterns. An embedding dimension of 6 was used for the mediolateral, 7 for the anteroposterior and 6 for the vertical direction. The $\mathrm{CoM}_{\text {vel }}$ data were normalised to 100 samples in length per stride. A time delay of 10 samples was chosen as all the time series had the same frequency. The slope of the divergence curve provided an estimate of the local divergence exponent. The local divergence exponent was calculated over the $\mathrm{CoM}_{\mathrm{vel}}$ in three directions. To overcome non-stationarities, velocity time series was used to estimate consistency of movement patterns [2]. 

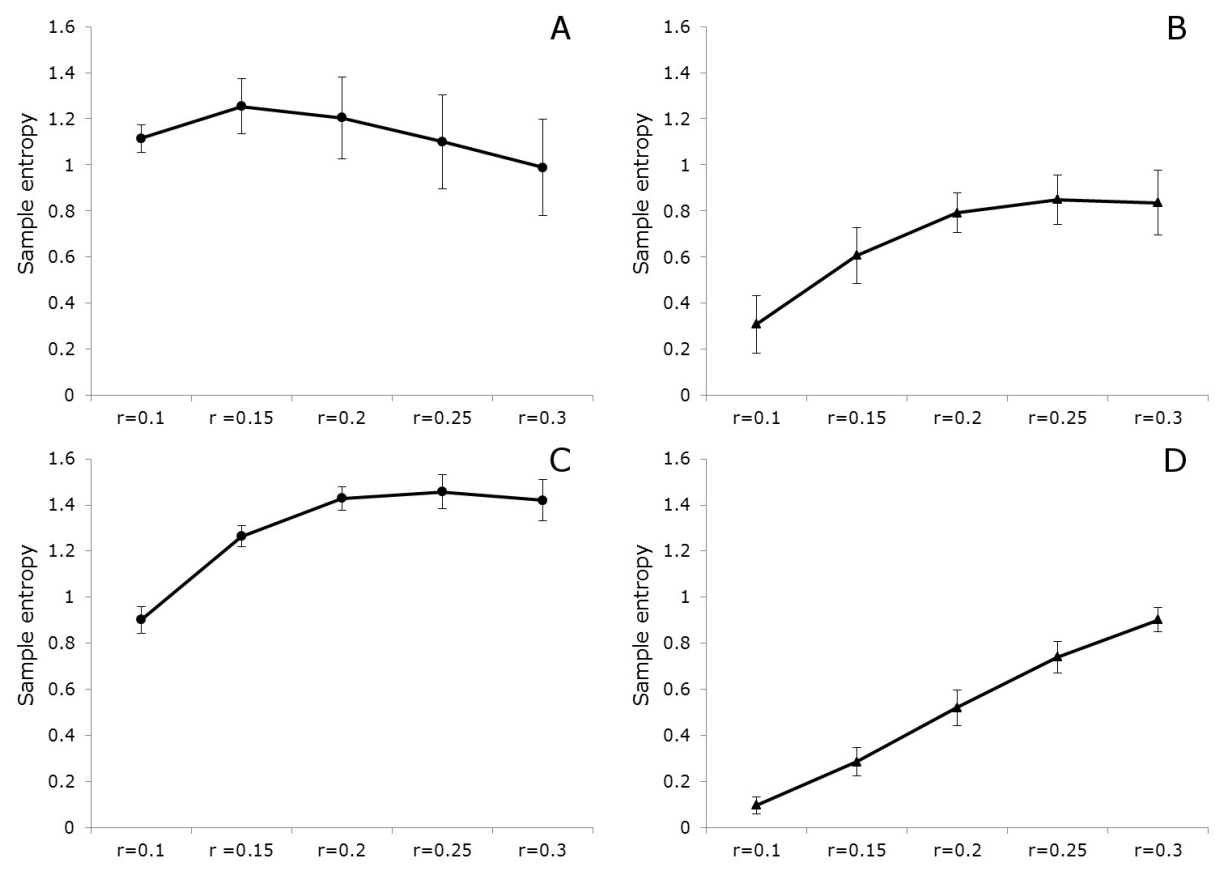

Figure 6.A.1. Sample entropy as a function of step length (A, B) and step width (C, D) for $r=0.1-0.3$, $m=2$ (left panel) and $m=3$ (right panel). 


\section{Bibliography}

1. Yentes, J. M. et al. The appropriate use of approximate entropy and sample entropy with short data sets. Ann Biomed Eng 41, 349-65 (2013).

2. Bruijn, S. M. et al. Assessing the stability of human locomotion: a review of current measures. $J R$ Soc Interface 10, 20120999 (2013). 

General discussion 


\section{Overview of findings}

The current thesis addressed the assessment of functional exercise capacity and gait characteristics in patients with chronic obstructive pulmonary disease (COPD). A self-paced, treadmill 6-minute walk test (6MWT) may provide additional advantages over the overground condition in clinical practice. The 6MWT on the Gait Real-time Analysis Interactive Lab (GRAIL) was found to be reproducible and valid in assessing the walk distance in both patients with COPD and healthy non-COPD subjects (Chapter 2). During the GRAIL-based 6MWT, patients with COPD demonstrated increased duration of temporal gait characteristics, decreased stride length and increased amount of stride-to-stride fluctuations as compared to non-COPD individuals (Chapter 3 ). The amount of fluctuations in stride length remained increased in COPD after correction for walking speed differences between the groups. However, reduced quadriceps strength was not associated with gait impairments, and COPD severity was only associated with swing time. Additional analyses were performed to assess the underlying patterns within stride-to-stride fluctuations in gait characteristics during the GRAIL-based 6MWT, which might unravel subtle alterations in gait that could not be assessed using traditional methods (Chapter 4). During the 6MWT, patterns within stride length fluctuations were more predictable in patients with COPD as compared to non-COPD subjects. Hip and knee joint movements were found to be locally less stable (i.e. less consistent organization of movement patterns) in patients with COPD when walking at three different walking speeds on a treadmill (Chapter 5). In addition, pulmonary rehabilitation resulted in patients taking faster steps, while no changes were observed in the amount of, and patterns within stride-to-stride fluctuations (Chapter 6). The current thesis provides a basis for further research in gait impairments in COPD in relation to balance and fall risk. These findings complement the understanding of gait impairments in COPD, which is important as COPD is recognized as a multisystemic disease affecting functional activities such as walking.

\section{Gait alterations in patients with COPD}

The ability of patients with COPD to perform activities of daily life necessary for independent living declines as their COPD progresses. Patients with COPD are therefore, more likely to experience disability [1]. Walking is a problematic activity in daily life in patients with COPD [2]. Though gait abnormalities have been associated with COPD severity [3], a thorough understanding of gait impairments in COPD and their associations with clinical parameters is lacking. 
Patients with COPD present with spatiotemporal gait alterations during the GRAIL-based 6MWT as compared to non-COPD subjects (Chapter 3 and Chapter 4). During the 6MWT, patients demonstrate slower cadence and shorter stride lengths, whereas temporal gait characteristics are increased [4]. These findings corroborate findings from previous studies, as patients with COPD have a reduced gait speed and a lower cadence measured over both short distances and during overground 6MWTs [5, 6]. However, when accounting for the differences in walking speed between the groups, patients were characterized with both increased stride length and amount of stride length fluctuations as compared to non-COPD subjects [7]. Yentes et al. [8] reported altered amount of stride-to-stride fluctuations in step time and step width in patients with COPD while walking at different walking speeds. Increased amount of stride-to-stride fluctuations may indicate a less cooperative behavior between the different components of the locomotor system, e.g. the central nervous system, muscles and joints, reflecting reduced gait function [9]. This may explain why patients with COPD report walking as problematic activity in daily life.

The temporal organization of gait variability (i.e. the patterns within stride-to-stride fluctuations in gait parameters) has been suggested to contain important information about the quality of movement [10]. Healthy individuals are characterized by a healthy movement variability, which may reflect a flexible locomotor system that is capable to adapt while walking. Less than healthy movement variability characterize a locomotor system that is overly rigid and unchanging, whereas greater than healthy variability characterizes a noisy and unstable locomotor system [9]. Both situations may characterize a locomotor system that is less adaptable to perturbations, as seen with aging, disease and individuals with increased fall risk [11-15]. Patients with COPD demonstrate altered patterns within stride-to-stride fluctuations during the GRAlL-based 6MWT as compared to non-COPD subjects (Chapter 4). Patterns within stride length fluctuations are more rigid in patients with COPD compared with non-COPD subjects. Similar patterns have been found in major physiological signals, such as airflow and heart rate patterns in COPD, indicating imbalances in its regulation [16]. In addition, knee and hip joint patterns were locally less stable in patients with COPD during treadmill walking across three different walking speeds (Chapter 5). Consequently, patients with COPD display less consistent organization of movement patterns in the lower extremities. Moreover, non-COPD subjects adjusted local dynamic stability of the movement patterns with increasing walking speed, whereas patients with COPD did not [17]. When walking at a faster pace, patients with COPD showed reduced local dynamic stability as compared to non-COPD subjects. These findings further strengthen the idea that patients with COPD have a less healthy gait function. This is in agreement with results reported in patients with peripheral arterial disease, who demonstrated less stable movement patterns as compared to healthy control subjects [15], indicating deterioration in the locomotor system of these patients. 
Furthermore, joint kinematics and kinetics are altered in patients with COPD as described previously by Yentes et al. [18]. Patients with COPD experience difficulties in lowering the forefoot after heel strike as compared to non-COPD subjects. Consequently, gait alterations are present in COPD at the joint level and could reflect impairments in other gait parameters. Deterioration in the patterns within stride-to-stride fluctuations indicates the presence of a less healthy locomotor system in patients with COPD, which could be related to functional limitations and increased fall risk in this patient group.

The 6MWT has proven to be an established clinical measure to determine functional exercise capacity, assess prognosis, and evaluate response to treatment [19]. Furthermore, the 6MWT is used for prescribing walking exercises for patients with COPD [20, 21]. The $6 \mathrm{MWT}$ is often used in clinical practice. Consequently, gait assessment combined with the 6MWT may be of added clinical value. The findings in this thesis support previous studies reporting reduced gait function in patients with COPD as compared to non-COPD subjects $[3,5,6,8,18,22-25]$. The differences in gait characteristics between patients with COPD and non-COPD control subjects were however small. This warrants for further research on methods to identify gait impairments in COPD and in turn the treatment of gait impairments in COPD.

More challenging gait protocols might be beneficial to assess gait impairments in COPD, than the ones that have been described in this thesis. Indeed, challenging conditions beyond normal walking for patients with COPD (e.g. faster or slower walking than the preferred-walking speed) may evoke alterations in gait characteristics more drastically [26]. A twenty percent change in the preferred walking speed as described in Chapter $\mathbf{5}$, may not be challenging enough to identify alterations in gait characteristics in patients with COPD. Larger changes in walking speed have been reported in the literature [27]. In addition, perturbation based gait assessment, the use of sudden expected mechanical perturbations during walking, is an emerging field in older adults to improve stepping response and fall risk [28]. Examples for perturbation based gait assessment are moveable floor platforms, ground surface changes, or treadmill belt accelerations or decelerations. Studies on perturbation based gait assessment showed improvements in reactive response to perturbations and reduction in falls in older adults [29-32]. Similar training and assessment protocols might be of interest in patients with COPD, as patients with COPD demonstrate gait impairments and are at increased risk for falls.

When cognitive and motor tasks are performed simultaneously, they compete for attentional resources [33] and information-processing neural pathways [34]. This may cause a decrease in the performance of one or both task (e.g. cognitive motor interference). Patients with COPD exhibited a greater increase in stride time variability than control subjects during dual task walking, indicating a clinical issue as such deficit increases the 
risk of falls [35]. Therefore, future research should examine various protocols to identify its clinical value for diagnostic, training and evaluation of treatment purposes for gait impairments in COPD.

\section{Mechanisms of gait impairments in COPD}

Gait alterations in COPD can be attributed to chronic respiratory limitations and the systemic comorbidities of the disease. COPD limits ventilation resulting in dynamic hyperinflation when expiratory time is not sufficient to enable lung emptying [36, 37]. This imposes constrains on tidal volume, leading to the increased sensation of dyspnea, the most common activity-limiting symptom of COPD. The level of dyspnea is correlated to the intensity of the patient's activity. Consequently, the patient with CODP tends to progressively reduce its physical activity.

Systemic inflammation is involved in the pathogenesis of most systemic effects of COPD [38], including skeletal muscle dysfunction [39]. Structural adaptations in muscles have been reported in COPD, in particular in the lower extremity of patients [40]. Sarcopenia and muscle atrophy, decreased capillary and mitochondrial density, and a shift from slow-twitch (type I) aerobic muscle fibers towards fast-twitch (type II) anaerobic muscle fibers, result in reduced oxidative and increased glycolytic capacity [41, 42]. The shift in fiber type can partially explain decreased endurance and a higher susceptibility to muscle fatigue and weakness in patients with COPD. In addition, physical inactivity leads to mechanical unloading that contributes to skeletal muscle abnormalities and dysfunction, such as loss of skeletal mass, size and strength, which ultimately lead to muscle atrophy. Indeed, patients with COPD are characterized by reduced quadriceps muscle strength and endurance (Chapter 3 ), confirming muscle weakness in thigh musculature in COPD [43]. In addition, muscle weakness of the ankle dorsi flexors and plantar flexors has been reported as well in patients with COPD [44, 45]. These muscle abnormalities indicate an overall decline in both the contractile and oxidative capacity of lower-limb muscles in patients with COPD, resulting in impaired functional exercise capacity and possibly gait function. However, mean spatiotemporal gait parameters did not differ between patients with COPD with reduced quadriceps muscle strength and those with high quadriceps muscle strength (Chapter 3).

Walking is a motor task requiring coordination of many muscles. For instance, the hip and knee extensors provide body support in early stance and the ankle plantar flexors are the primary contributors in late stance. Forward propulsion (defined as horizontal acceleration of the body center of mass) in walking is provided by the hip and knee ex- 
tensors in early stance and ankle plantar flexors in late stance. In addition, the plantar flexors are essential to walking subtasks such as swing initiation and power generation in healthy walking [46-48]. Furthermore, sensorimotor factors, such as body sway, reaction time and proprioception, have been considered as likely factors that may explain gait variability in the general older population aged 60-86 years old [49]. These factors have not been assessed in the current thesis. Future studies should therefore be directed to identifying specific contributors to gait and gait impairments in patients with COPD. Another explanation for the lack of influence of quadriceps muscle strength on gait characteristics could be that patients with COPD are very heterogeneous and the number of subjects was small in the subgroups described in Chapter 3. Therefore, this analysis may lack statistical power to differentiate gait characteristics between the subgroups.

\section{Balance and falls in COPD}

Falls are common among older individuals and can have devastating effects on overall function, health related quality of life and life expectancy. Chronic conditions, including COPD, have been associated with a higher risk for falls [50]. Patients with COPD are 55\% more likely to have an incident record of a fall than non-COPD subjects [51], which is associated with a worsening of dyspnea perception as related to health related quality of life [52]. The causes of falls are multifactorial, however, most of them happen during walking [53]. Impaired gait function is therefore one of the main risk of falls in older adults [54]. Consequently, it is not surprising that gait impairments in COPD have been suggested to be related to impaired balance control, and in turn with increased fall risk in COPD [55-59].

Several gait parameters have been associated with falls, including changes in stride-tostride fluctuations [11, 49, 60-63]. Walking reflects the displacement of the center of mass of the human body and the center of mass displacement in the mediolateral direction has been related to stability and falls [64]. Indeed, patients with peripheral arterial disease have less stable joint movements during walking [15], and it has been suggested to be a valid measure to estimate the probability of falling [11, 61]. However, patients with COPD did not demonstrated reduced local dynamic stability of the center of mass displacement during the GRAIL-based 6MWT (Chapter 4), whereas local dynamic stability in the lower extremity was reduced in patients with COPD. Reduced long-range correlations have been suggested to be related to balance, especially in mediolateral direction. In addition, lower walking intensity and increased variation in mediolateral trunk movements were observed in patients with COPD during an overground 6MWT [5]. Increased variation in mediolateral trunk movements in COPD suggests larger balance disturbances 
during the 6MWT in these patients. This may at least partially contribute to the relative high energetic costs of a 6MWT in COPD [65]. Moreover, this may also explain partially why patients with COPD experience abnormalities with walking and increased risk for falls $[2,52]$. Similar deviations in gait were previously observed in frail elderly, who fell at least once during the last year or who used walking aids [63]. Furthermore, increased stride length variability in patients with COPD (Chapter 3 and Chapter 4 ) has been suggested to be associated with increased fall risk and balance disturbances in the aging population [62].

Risk factors for falls such as lower limb muscle weakness and deficits in functional performance and postural control are common in individuals with COPD. In general, the use of proprioception is of primary importance during balance control. Patients with COPD, especially those with inspiratory muscle weakness, increased their reliance on ankle muscle proprioceptive signals and decreased their reliance on back muscle proprioceptive signals during balance control, resulting in a decreased postural stability compared to healthy controls [66]. Furthermore, patients with COPD have worse static and functional balance in comparison with non-COPD subjects [67]. The ability to maintain stable is critical for functional independence in activities in daily living, mobility and for avoiding falls. It seems that falls and gait are associated. Therefore, additional studies are recommended to identify a mechanistic link between gait impairments and falls in patients with COPD.

\section{The effects of pulmonary rehabilitation on gait characteristics in COPD}

The goals of pulmonary rehabilitation (PR) include minimizing symptom burden, maximizing exercise performance, promoting autonomy, increasing participation in everyday activities, enhancing health-related quality of life, and effecting long-term healthenhancing behavior change [68]. PR has demonstrated improvements in symptoms, exercise tolerance, and health related quality of life in patients with COPD. Exercise capacity in patients with COPD is impaired and exercise training as part of a comprehensive pulmonary rehabilitation program is used to improve muscle function in COPD [69-71]. Chapter 6 provided evidence of increased quadriceps muscle strength, muscle endurance and fat free mass in patients with COPD following PR. In addition, patients with COPD showed improvements in functional tests, such as the timed up and go test and both the overground and GRAIL-based 6MWT. Patients reported less fatigue at the overground 6MWT after PR, while a larger distance was covered. During the GRAIL-based 6MWT, patients reported lower end values of dyspnea after PR as compared to before PR. These 
findings confirm that PR improves functional exercise capacity and symptoms in COPD.

Chapter 6 showed that patients walked faster during the GRAIL-based 6MWT after PR, with reduced mean stride time and a trend of increased stride length. On the contrary of what was hypothesized, an increase in quadriceps muscle strength and whole body fat free mass did not translate to improvements in the stride-to-stride fluctuations in patients with COPD. Consequently, PR does not directly relate to a better locomotor system, as has been found in community dwelling older adults following an exercise training program [72]. In addition, the response to PR may vary substantially between patients with COPD, and individual patients may respond differently on various types of outcome measures [73]. When patients with COPD were divided into good responders and poor responders based on the improvement in walk distance on the GRAIL, differences were found in the overground 6MWD. Walking speed was higher in the good responders, with shorter stride times and larger stride lengths. However, differences in the stride-to-stride fluctuations in gait characteristics were not observed. This indicates that improvements in walking distance and gait speed following PR, does not necessarily translate into improvements in the stride-to-stride fluctuations in gait in patients with COPD.

The current training program of the PR targeted large muscle groups of the body and was not aimed at smaller muscle groups that have a significant contribution to gait and balance function. This could explain partially why less improvement in stride-to-stride fluctuations was found after PR in patients with COPD. Though patients with COPD had treadmill-based exercise training, gait training was not provided in the current training program, e.g. dual task or perturbation based training. This might explain why patients with COPD were not exposed to challenging environments that could have improved their locomotor system towards more healthy gait variability and its fluctuations, that is more likely to adapt to changes on the body.

General exercise interventions (combinations of strength, balance and aerobic exercises) have generally resulted in moderate reductions (14-17\%) in falls incidence among older adults [74-76]. These exercise interventions often lack specificity to the balance recover mechanisms that are needed following balance loss, such as compensatory stepping, counter rotation or grasping actions $[62,77]$. Balance maintenance requires a complex interaction of several mechanisms and improved balance control in one task might not be of benefit during other tasks [78]. Therefore, training aimed more specifically at balance recovery mechanisms may provide additional insight and benefit for falls reduction and prevention in general. This could be of interest in patients with COPD, as previous studies documented impairments in clinical and laboratory measures of balance in patients with COPD compared to non-COPD control subjects and the associated between balance and fall risk [52, 55, 57, 66, 79-82]. The current training program during PR did not specifi- 
cally provide balance training to patients with COPD. Therefore, balance training might be considered as an additional training modality in patients with COPD that could be of interest to improve gait function as well.

\section{Is the GRAIL-based 6-minute walk test a replacement for the overground 6-minute walk test in patients with COPD?}

Exercise intolerance is an important clinical feature in patients with COPD, as it correlates with impaired quality of life $[83,84]$. In clinical practice, the 6MWT is a measure to evaluate functional exercise capacity in patients with COPD [19]. The 6MWT is recommended to be administered in a flat corridor of at least 30 meters by the American Thoracic Society and European Respiratory Society, whereas externally paced treadmill testing is not recommended [85]. Two studies [86, 87] were mentioned that reported $13-20 \%$ lower distances covered on a treadmill as compared to overground. This discrepancy could be explained by poor walking efficiency during treadmill walking in subjects unaccustomed to this activity. However, the GRAIL uses the novel approach of a virtual reality-based selfpaced treadmill, which integrates a self-paced algorithm to regulate the treadmill's speed [88]. Consequently, subjects are able to select and change their own walking speed. It has been reported to result in long-term fluctuations that resemble those as seen during overground walking [89]. In addition, small differences between self-paced treadmill walking in a virtual reality and walking in a conventional gait lab were reported previously [88]. To account for this matter, subjects are recommended to undergo a familiarization session prior to the two GRAIL-based 6MWTs to become accustomed to the GRAIL.

The GRAIL requires limited space and provides a safe environment for the patient. This is in contrast to the overground 6MWT that requires at least 30 meters in a clinical setting, in which the patient might be disturbed by passers-by or have limited space to turn. In addition, the GRAIL's track layout and length are similar for all subjects on subsequent occasions, as these factors affect performance on the test as well [19]. Furthermore, subjects have reported walking with the virtual reality as more similar to overground walking [90], further encouraging the use of this system.

Based on the main outcome parameter of the 6MWT, there is still a discrepancy in the walk distances between the overground and GRAIL 6MWT. Patients tend to walk less far on the GRAIL as compared to overground (Chapter 2). Furthermore, the GRAIL would not be available to all clinical sites, as this system is more expensive as compared to the simple overground 6MWT. However, the GRAIL enables additional possibilities that could not be assessed in a clinical setting during an overground 6MWT. Continuous strides and gait 
patterns could be evaluated using the 3-dimensional motion analysis and force plates, obtaining spatiotemporal, kinematic and kinetic parameters. Moreover, all steps taken by the subject could be recorded, which is of importance to obtain an accurate insight of the walking ability of the patient, in particular during exercise testing.

Based on Chapter 2, we can conclude that the GRAIL is a promising system to assess the 6MWD reliably and validly in patients with COPD and non-COPD subjects as compared to previous studies [86, 87]. Though the GRAIL underestimates the walk distance covered overground, the GRAIL should be considered if the walking ability of the patient is impaired and a thorough assessment of the patient's gait is needed.

\section{Further implications of the GRAIL}

Clinical gait analysis is a well-established instrument for the objective assessment of gait. Such analysis allows to identify specific gait abnormalities to recommend treatment program and monitor the effect of a treatment. The GRAIL system has been used for gait assessment in various patient populations, including COPD [4], cerebral palsy [91], diabetic peripheral neuropathy [92], and stroke [93]. Within a safe environment, multiple gait cycles can be obtained, increasing data reliability [94], and allows for the evaluation of stride-to-stride fluctuations and changes over time.

The GRAIL may present challenging conditions to the patient by induced gait perturbations, as well as the manipulation of visual and proprioceptive feedback. Consequently, these challenging environments to examine functional gait may be a more revealing method to assess movement pathologies and fall risk. The GRAIL is therefore a promising tool for training purposes and clinical gait analysis in the field of rehabilitation. Exercises can be individualized to the patient's ability and real-time feedback on gait characteristics can be provided. In addition, different aspects of gait and balance can be addressed using various challenging conditions. As a result, the ability of patients to adapt their gait can be assessed, providing information on compensatory mechanisms [95].

It has been shown that balance and gait training on the GRAIL is feasible in patients after stroke [96]. Consequently, GRAIL-based training may benefit patients with COPD as well. Given the high risk of falls and disability associated with altered stride-to-stride fluctuations [11, 60-63, 97], research on challenging conditions and gait analysis in COPD are recommended. This may provide evidence and guidance for future training modalities, such as dual task interventions and perturbations based training, in pulmonary rehabilitation programs. In addition, the GRAIL can be used for real-life mimicking tasks as patients with COPD experience problems during activities in daily life. Furthermore, the symptoms of 
COPD, including fatigue and dyspnea, often interfere with activities of daily life, leading to a significant reduction in quality of life [98-102]. Therefore, research on the influence of fatigue or dyspnea on gait characteristics and balance disturbances is highly valuable. Given the increased energy expenditure in patients with COPD during activities in daily life [103], the assessment of walking economy using the GRAIL may be considered.

\section{Limitations}

Some limitations should be acknowledged when interpreting the results of this thesis. This thesis presented the first results on obtaining the 6MWD using the GRAIL-system, which combined self-paced treadmill walking with 3D motion analysis within a virtual reality environment. The effect of optic flow provided by the virtual reality environment was not examined in the specific patient or age group. This could have presented a challenge or helped those due to the increase in visual information during walking. A previous study assessed self-paced treadmill walking and virtual reality in healthy young adults [90]. The effects of virtual reality on gait were too small to be relevant and subjects reported walking with virtual reality as more similar to overground walking.

In addition, the adaptation to self-paced treadmill walking might have been difficult to patients with COPD. Due to the symptoms patients with COPD experience and their limited exercise capacity, they may not have been completely accustomed to adjust their walking speed, especially during prolonged exercise testing such as during the 6MWT. However, patients walked a minimum of 3 minutes to familiarize with self-paced treadmill walking. Different regulation methods were observed to control the treadmill speed in patients with COPD and non-COPD subjects. However, due to practical issues and the physical condition of the patients in particular, we did not prolong or add additional familiarization sessions. In addition, self-paced treadmill walking has only been studied in healthy subjects [89] and in patients with cerebral palsy [88].

Walking speed is of influence on gait characteristics and is an important factor to control when interpreting the data [27, 104, 105]. Nowadays, there are different options to assess gait while walking on a treadmill. For instance, walking at a fixed speed, at a preferred walking speed or self-paced walking. When comparing different groups, researchers should consider comparing the groups at a comparable walking speed or while each group is walking in a similar condition (e.g. at their preferred walking speed or in a similar challenging condition). A clear rationale should be presented and a careful consideration is necessary a priori.

Patients with COPD included in the studies were mainly categorized as GOLD II and III. We 
acknowledge that it has implications on the generalizability of our findings. This limitation was foremost due to practical issues, such that patients with COPD in GOLD I were less often referred to pulmonary rehabilitation, whereas patients with COPD in GOLD IV were not able to walk independently, without walking aids, on the treadmill for a prolonged period of time.

Spatiotemporal variability measures have strong to moderate construct, predictive, convergent, and predictive validity of falling, whereas most measures to quantify the patterns within stride-to-stride fluctuations do not [11]. Therefore, future studies should provide evidence for the theoretic framework of movement variability and health as has been proposed by Stergiou et al. [106, 107]. In particular, stride-to-stride fluctuations have not been investigated in patients with COPD. Patients with COPD are at increased risk for falls and falls often occur during walking. Therefore, patients with COPD might benefit from research on identifying parameters for gait impairments in this specific population.

\section{Recommendations for future studies and clinical practice}

While this thesis provides new insights in gait characteristics in patients with COPD, several important questions remain to be investigated. Patients with COPD demonstrate functional limitations and gait alterations; they also show reduced balance control and increased fall risk. However, the underlying mechanisms and relationship between these parameters have not been investigated thoroughly. This thesis provided the first results on gait alterations in COPD using extensive parameters that have been associated with fall risk and balance disturbances in other populations. Future studies are therefore recommended to identify the interrelationships between these parameters in COPD.

As research in gait in COPD has gained interest, clinical relevant changes in gait characteristics in this specific patient population should be investigated to provide clinical support when assessing gait function in COPD. Though, meaningful changes in the amount of stride-to-stride fluctuations have been reported in older adults [108], it has not been reported in measures of the patterns within stride-to-stride fluctuations. This remains to be explored and determined in the aging population as well as in pathological populations such as COPD.

Another question that needs to be addressed is the effect of gait and/or balance training on gait characteristics in patients with COPD. Research on whether the effects of gait training on gait function is beneficial or not should be performed. Limited research has been conducted in assessing gait stride-to-stride fluctuations following a certain training program. This calls for future studies in providing insight into the effects of different 
training modalities on gait stride-to-stride fluctuations in the aging population and pathological populations including COPD.

Walking impairments may arise more strongly when exposing patients with COPD to perturbations during walking. Moreover, impaired ability to respond accordingly to perturbations could be associated with increased fall risk in this patient population. Slipping and tripping during walking are the most common causes of falls among older adults, which represent failures to adapt to changes and challenges in the environment [28]. Future recommendations are to explore the relationship between dynamic balance and gait in patients with COPD. For example, to study the effects of perturbation based exercises on patients with COPD.

\section{Concluding remarks}

This thesis demonstrated that patients with COPD have altered spatiotemporal gait characteristics and stride-to-stride fluctuations as compared to non-COPD subjects. The amount of stride-to-stride fluctuations in stride length and stride length regularity showed to be sensitive to differentiate patients with COPD and non-COPD subjects during the GRAILbased 6MWT, when accounting for walking speed differences between the two groups. Local dynamic stability in the hip and knee joint is reduced in patients with COPD when walking at different walking speeds and patients demonstrated fewer changes in local dynamic stability as compared to non-COPD subjects. Though the current training program in pulmonary rehabilitation improves skeletal muscle function and exercise capacity, it does not result in improvements in the locomotor system in terms of gait variability. Therefore, additional training programs in pulmonary rehabilitation, e.g. training specifically designed for improving gait function, might be beneficial to gain improvements in mobility, balance and falls in patients with COPD. The GRAIL has proven to be of clinical value and may be used for future studies on gait function in COPD. 


\section{Bibliography}

1. Kanervisto, M. et al. COPD, chronic bronchitis and capacity for day-to-day activities: negative impact of illness on the health-related quality of life. Chron Respir Dis 7, 207-15 (2010).

2. Annegarn, J. et al. Problematic activities of daily life are weakly associated with clinical characteristics in COPD. J Am Med Dir Assoc 13, 284-90 (2012).

3. Yentes, J. M. et al. Walking abnormalities are associated with COPD: An investigation of the NHANES III dataset. Respir Med 105, 80-7 (2011).

4. Liu, W. Y. et al. Spatiotemporal gait characteristics in patients with COPD during the Gait Real-time Analysis Interactive Lab-based 6-minute walk test. PLoS One 12, e0190099 (2017).

5. Annegarn, J. et al. Differences in walking pattern during 6-min walk test between patients with COPD and healthy subjects. PLoS One 7, e37329 (2012).

6. Lahousse, L. et al. Gait patterns in COPD: the Rotterdam Study. Eur Respir J 46, 88-95 (2015).

7. Liu, W. et al. Stride-to-stride fluctuations in patients with COPD during the self-paced treadmill 6-minute walk test. (Submitted).

8. Yentes, J. M. et al. Patients with Chronic Obstructive Pulmonary Disease Walk with Altered Step Time and Step Width Variability as Compared with Healthy Control Subjects. Ann Am Thorac Soc 14, 858-866 (2017).

9. Stergiou, N., Harbourne, R. \& Cavanaugh, J. Optimal movement variability: a new theoretical perspective for neurologic physical therapy. J Neurol Phys Ther 30, 120-9 (2006).

10. Harbourne, R. T. et al. Complexity of postural control in infants: linear and nonlinear features revealed by principal component analysis. Nonlinear Dynamics Psychol Life Sci 13, 123-44 (2009).

11. Bruijn, S. M. et al. Assessing the stability of human locomotion: a review of current measures. J R Soc Interface 10, 20120999 (2013).

12. Buzzi, U. H. et al. Nonlinear dynamics indicates aging affects variability during gait. Clin Biomech (Bristol, Avon) 18, 435-43 (2003).

13. Goldberger, A. L. et al. Fractal dynamics in physiology: alterations with disease and aging. Proc Natl Acad Sci U S A 99 Suppl 1, 2466-72 (2002).

14. Hausdorff, J. M. et al. Altered fractal dynamics of gait: reduced stride-interval correlations with aging and Huntington's disease. J Appl Physiol (1985) 82, 262-9 (1997).

15. Myers, S. A. et al. Gait variability is altered in patients with peripheral arterial disease. J Vasc Surg 49, 924-931 e1 (2009).

16. Jin, Y. et al. Entropy change of biological dynamics in COPD. Int J Chron Obstruct Pulmon Dis 12, 29973005 (2017).

17. Liu, W. et al. Patients with COPD walk with less consistent organization of movement patterns of the lower extremity. (Submitted).

18. Yentes, J. M. et al. Gait mechanics in patients with chronic obstructive pulmonary disease. Respir Res 16, 31 (2015).

19. Holland, A. E. et al. An official European Respiratory Society/American Thoracic Society technical standard: field walking tests in chronic respiratory disease. Eur Respir J 44, 1428-46 (2014).

20. Jenkins, S. C. 6-Minute walk test in patients with COPD: clinical applications in pulmonary rehabilitation. Physiotherapy 93, 175-182 (2007).

21. Rodrigues, A. et al. Is the six-minute walk test a useful tool to prescribe high-intensity exercise in patients with chronic obstructive pulmonary disease? Heart Lung 45, 550-556 (2016).

22. McCamley, J. D. et al. Gait deficiencies associated with peripheral artery disease are different than chronic obstructive pulmonary disease. Gait Posture 57, 258-264 (2017).

23. Nantsupawat, N. et al. Gait Characteristics in Patients With Chronic Obstructive Pulmonary Disease. J Prim Care Community Health 6, 222-6 (2015). 
24. Rutkowski, S. et al. Gait analysis of patients with chronic obstructive pulmonary disease 24-33 (2014).

25. Zago, M. et al. Gait analysis in patients with chronic obstructive pulmonary disease: a systematic review. Gait Posture 61, 408-415 (2018).

26. Helbostad, J. L. \& Moe-Nilssen, R. The effect of gait speed on lateral balance control during walking in healthy elderly. Gait Posture 18, 27-36 (2003).

27. Chien, J. H. et al. The Effect of Walking Speed on Gait Variability in Healthy Young, Middle-aged and Elderly Individuals. J Phys Act Nutr Rehabil 2015 (2015).

28. McCrum, C. et al. A systematic review of gait perturbation paradigms for improving reactive stepping responses and falls risk among healthy older adults. Eur Rev Aging Phys Act 14, 3 (2017).

29. Bhatt, T., Yang, F. \& Pai, Y. C. Learning to resist gait-slip falls: long-term retention in community-dwelling older adults. Arch Phys Med Rehabil 93, 557-64 (2012).

30. Lurie, J. D. et al. Pilot comparative effectiveness study of surface perturbation treadmill training to prevent falls in older adults. BMC Geriatr 13, 49 (2013).

31. Pai, Y. C. et al. Inoculation against falls: rapid adaptation by young and older adults to slips during daily activities. Arch Phys Med Rehabil 91, 452-9 (2010).

32. Pai, Y. C. et al. Learning from laboratory-induced falling: long-term motor retention among older adults. Age (Dordr) 36, 9640 (2014).

33. Wickens, C. D. The structure of attentional resources. Attention and performance VIII 8, 239-257 (1980).

34. Friedman, A. et al. Dividing attention within and between hemispheres: testing a multiple resources approach to limited-capacity information processing. J Exp Psychol Hum Percept Perform 8, 625-50 (1982).

35. Heraud, N. et al. Impact of Chronic Obstructive Pulmonary Disease on Cognitive and Motor Performances in Dual-Task Walking. COPD, 1-6 (2018).

36. Maclntyre, N. R. Mechanisms of functional loss in patients with chronic lung disease. Respir Care 53, 1177-84 (2008).

37. O'Donnell, D. E. et al. Mechanisms of activity-related dyspnea in pulmonary diseases. Respir Physiol Neurobiol 167, 116-32 (2009).

38. Agusti, A. Systemic effects of chronic obstructive pulmonary disease: what we know and what we don't know (but should). Proc Am Thorac Soc 4, 522-5 (2007).

39. Global strategy for the diagnosis, management, and prevention of COPD Web Page. Accessed: 27-072018. http: //goldcopd.org/ (Global Initiative for Chronic Obstructive Lung Disease).

40. Gosselink, R., Troosters, T. \& Decramer, M. Peripheral muscle weakness contributes to exercise limitation in COPD. Am J Respir Crit Care Med 153, 976-80 (1996).

41. Kim, H. C., Mofarrahi, M. \& Hussain, S. N. Skeletal muscle dysfunction in patients with chronic obstructive pulmonary disease. Int J Chron Obstruct Pulmon Dis 3, 637-58 (2008).

42. Mador, M. J. \& Bozkanat, E. Skeletal muscle dysfunction in chronic obstructive pulmonary disease. Respir Res 2, 216-24 (2001).

43. Seymour, J. M. et al. The prevalence of quadriceps weakness in COPD and the relationship with disease severity. Eur Respir J 36, 81-8 (2010).

44. Gagnon, P. et al. Distal leg muscle function in patients with COPD. COPD 10, 235-42 (2013).

45. Maddocks, M. et al. Ankle dorsiflexor muscle size, composition and force with ageing and chronic obstructive pulmonary disease. Exp Physiol 99, 1078-88 (2014).

46. Anderson, F. C. \& Pandy, M. G. Individual muscle contributions to support in normal walking. Gait Posture 17, 159-69 (2003).

47. Liu, M. Q. et al. Muscles that support the body also modulate forward progression during walking. $J$ Biomech 39, 2623-30 (2006).

48. Neptune, R. R., Zajac, F. E. \& Kautz, S. A. Muscle force redistributes segmental power for body progression during walking. Gait Posture 19, 194-205 (2004). 
49. Callisaya, M. L. et al. Sensorimotor factors affecting gait variability in older people-a population-based study. J Gerontol A Biol Sci Med Sci 65, 386-92 (2010).

50. Lawlor, D. A., Patel, R. \& Ebrahim, S. Association between falls in elderly women and chronic diseases and drug use: cross sectional study. BMJ 327, 712-7 (2003).

51. Hakamy, A. et al. Risk of fall in patients with COPD. Thorax 73, 1079-1080 (2018).

52. Roig, M. et al. Falls in people with chronic obstructive pulmonary disease: an observational cohort study. Respir Med 105, 461-9 (2011).

53. Bridenbaugh, S. A. \& Kressig, R. W. Laboratory review: the role of gait analysis in seniors' mobility and fall prevention. Gerontology 57, 256-64 (2011).

54. Verghese, J. et al. Quantitative gait markers and incident fall risk in older adults. J Gerontol A Biol Sci Med Sci 64, 896-901 (2009).

55. Beauchamp, M. K. et al. Impairments in systems underlying control of balance in COPD. Chest 141, 1496503 (2012).

56. Hellstrom, K. et al. Fear of falling, fall-related self-efficacy, anxiety and depression in individuals with chronic obstructive pulmonary disease. Clin Rehabil 23, 1136-44 (2009).

57. Oliveira, C. C. et al. Falls by individuals with chronic obstructive pulmonary disease: a preliminary 12month prospective cohort study. Respirology 20, 1096-101 (2015).

58. Roig, M. et al. Deficits in muscle strength, mass, quality, and mobility in people with chronic obstructive pulmonary disease. J Cardiopulm Rehabil Prev 31, 120-4 (2011).

59. Smith, M. D. et al. Balance is impaired in people with chronic obstructive pulmonary disease. Gait \& Posture 31, 456-60 (2010).

60. Brach, J. S. et al. Too much or too little step width variability is associated with a fall history in older persons who walk at or near normal gait speed. J Neuroeng Rehabil 2, 21 (2005).

61. Lockhart, T. E. \& Liu, J. Differentiating fall-prone and healthy adults using local dynamic stability. Ergonomics 51, 1860-72 (2008).

62. Maki, B. E. Gait changes in older adults: predictors of falls or indicators of fear. J Am Geriatr Soc 45, 313-20 (1997).

63. Moe-Nilssen, R. \& Helbostad, J. L. Interstride trunk acceleration variability but not step width variability can differentiate between fit and frail older adults. Gait \& Posture 21, 164-70 (2005).

64. Weinert-Aplin, R. A. et al. Medial-lateral centre of mass displacement and base of support are equally good predictors of metabolic cost in amputee walking. Gait Posture 51, 41-46 (2017).

65. Troosters, T. et al. Physiological responses to the 6-min walk test in patients with chronic obstructive pulmonary disease. Eur Respir J 20, 564-9 (2002).

66. Janssens, L. et al. Proprioceptive changes impair balance control in individuals with chronic obstructive pulmonary disease. PLoS One 8, e57949 (2013).

67. De Castro, L. A. et al. Static and Functional Balance in Individuals With COPD: Comparison With Healthy Controls and Differences According to Sex and Disease Severity. Respir Care 61, 1488-1496 (2016).

68. Spruit, M. A. et al. An official American Thoracic Society/European Respiratory Society statement: key concepts and advances in pulmonary rehabilitation. Am J Respir Crit Care Med 188, e13-64 (2013).

69. Bernard, S. et al. Aerobic and strength training in patients with chronic obstructive pulmonary disease. Am J Respir Crit Care Med 159, 896-901 (1999).

70. Maltais, F. et al. Skeletal muscle adaptation to endurance training in patients with chronic obstructive pulmonary disease. Am J Respir Crit Care Med 154, 442-7 (1996).

71. Man, W. D. et al. Symptoms and quadriceps fatigability after walking and cycling in chronic obstructive pulmonary disease. Am J Respir Crit Care Med 168, 562-7 (2003).

72. Wang, R. Y. et al. Effects of combined exercise on gait variability in community-dwelling older adults. Age (Dordr) 37, 9780 (2015). 
73. Spruit, M. A. et al. Differential response to pulmonary rehabilitation in COPD: multidimensional profiling. Eur Respir J 46, 1625-35 (2015).

74. Chang, J. T. et al. Interventions for the prevention of falls in older adults: systematic review and metaanalysis of randomised clinical trials. BMJ 328, 680 (2004).

75. Gillespie, L. D. et al. Interventions for preventing falls in older people living in the community. Cochrane Database Syst Rev, CD007146 (2012).

76. Sherrington, C. et al. Effective exercise for the prevention of falls: a systematic review and meta-analysis. J Am Geriatr Soc 56, 2234-43 (2008).

77. Hof, A. L. The equations of motion for a standing human reveal three mechanisms for balance. J Biomech 40, 451-7 (2007).

78. Kummel, J. et al. Specificity of Balance Training in Healthy Individuals: A Systematic Review and MetaAnalysis. Sports Med 46, 1261-71 (2016).

79. Beauchamp, M. K., Brooks, D. \& Goldstein, R. S. Deficits in postural control in individuals with COPD emerging evidence for an important secondary impairment. Multidiscip Respir Med 5, 417-21 (2010).

80. Beauchamp, M. K. et al. Impairments in balance discriminate fallers from non-fallers in COPD. Respir Med 103, 1885-91 (2009).

81. Butcher, S. J., Meshke, J. M. \& Sheppard, M. S. Reductions in functional balance, coordination, and mobility measures among patients with stable chronic obstructive pulmonary disease. J Cardiopulm Rehabil 24, 274-80 (2004).

82. Janssens, L. et al. Impaired postural control reduces sit-to-stand-to-sit performance in individuals with chronic obstructive pulmonary disease. PLoS One 9, e88247 (2014).

83. Brown, C. D. et al. Exercise testing in severe emphysema: association with quality of life and lung function. COPD 5, 117-24 (2008).

84. Oga, T. et al. Relationship between different indices of exercise capacity and clinical measures in patients with chronic obstructive pulmonary disease. Heart Lung 31, 374-81 (2002).

85. Singh, S. J. et al. An official systematic review of the European Respiratory Society/American Thoracic Society: measurement properties of field walking tests in chronic respiratory disease. Eur Respir J 44, 1447-78 (2014).

86. De Almeida, F. G., Victor, E. G. \& Rizzo, J. A. Hallway versus treadmill 6-minute-walk tests in patients with chronic obstructive pulmonary disease. Respir Care 54, 1712-6 (2009).

87. Stevens, D. et al. Comparison of hallway and treadmill six-minute walk tests. Am J Respir Crit Care Med 160, 1540-3 (1999).

88. Van der Krogt, M. M., Sloot, L. H. \& Harlaar, J. Overground versus self-paced treadmill walking in a virtual environment in children with cerebral palsy. Gait \& Posture 40, 587-93 (2014).

89. Sloot, L. H., van der Krogt, M. M. \& Harlaar, J. Self-paced versus fixed speed treadmill walking. Gait \& Posture 39, 478-84 (2014).

90. Sloot, L. H., van der Krogt, M. M. \& Harlaar, J. Effects of adding a virtual reality environment to different modes of treadmill walking. Gait \& Posture 39, 939-45 (2014).

91. Booth, A. T. et al. Immediate Effects of Immersive Biofeedback on Gait in Children With Cerebral Palsy. Archives of Physical Medicine and Rehabilitation 100, 598-605 (2019).

92. Huang, C.-K. et al. An altered spatiotemporal gait adjustment during a virtual obstacle crossing task in patients with diabetic peripheral neuropathy. Journal of Diabetes and its Complications 33, 182-188 (2019).

93. Punt, M. et al. Virtual obstacle crossing: Reliability and differences in stroke survivors who prospectively experienced falls or no falls. Gait \& Posture 58, 533-538 (2017).

94. Monaghan, K., Delahunt, E. \& Caulfield, B. Increasing the number of gait trial recordings maximises intra-rater reliability of the CODA motion analysis system. Gait Posture 25, 303-15 (2007).

95. Van Gelder, L. et al. Real-time feedback to improve gait in children with cerebral palsy. Gait Posture 52, 76-82 (2017). 
96. De Rooij, I., van de Port, I. \& Meijer, J.-W. Feasibility and Effectiveness of Virtual Reality Training on Balance and Gait Recovery Early after Stroke: A Pilot Study. Int J Phys Med Rehabil 5, 1-8 (2017).

97. Hausdorff, J. M., Rios, D. A. \& Edelberg, H. K. Gait variability and fall risk in community-living older adults: a 1-year prospective study. Arch Phys Med Rehabil 82, 1050-6 (2001).

98. Andersson, M. et al. Physical activity and fatigue in chronic obstructive pulmonary disease - A population based study. Respiratory Medicine 109, 1048-1057 (2015).

99. Breslin, E. et al. Perception of Fatigue and Quality of Life in Patients With COPD. Chest 114, 958-964 (1998).

100. Katajisto, M. et al. Physical inactivity in COPD and increased patient perception of dyspnea. International journal of chronic obstructive pulmonary disease 7, 743-755 (2012).

101. Kouijzer, M., Brusse-Keizer, M. \& Bode, C. COPD-related fatigue: Impact on daily life and treatment opportunities from the patient's perspective. Respiratory Medicine 141, 47-51 (2018).

102. Lahaije, A. J. et al. Physiologic limitations during daily life activities in COPD patients. Respir Med 104, 1152-9 (2010).

103. Patel, S. A. et al. Activity Monitoring and Energy Expenditure in COPD Patients: A Validation Study. COPD: Journal of Chronic Obstructive Pulmonary Disease 4, 107-112 (2007).

104. England, S. A. \& Granata, K. P. The influence of gait speed on local dynamic stability of walking. Gait Posture 25, 172-8 (2007).

105. Jordan, K., Challis, J. H. \& Newell, K. M. Walking speed influences on gait cycle variability. Gait \& Posture 26, 128-34 (2007).

106. Stergiou, N. Nonlinear Analysis for Human Movement Variability (CRC Press, Boca Raton, 2016).

107. Stergiou, N. \& Decker, L. M. Human movement variability, nonlinear dynamics, and pathology: is there a connection? Hum Mov Sci 30, 869-88 (2011).

108. Brach, J. S. et al. Meaningful change in measures of gait variability in older adults. Gait Posture $31,175-9$ (2010). 
Summary 
Chronic obstructive pulmonary disease (COPD) is characterized by persistent airflow limitation, which causes symptoms of breathlessness and fatigue. Moreover, COPD is recognized as a multisystemic disease, affecting the neuromuscular system as well. Patients with COPD have a reduced functional exercise capacity and limited mobility as the disease progresses. Consequently, patients become limited in activities of daily living. Walking is a problematic activity in patients' daily life. This could be associated with gait impairments in patients with COPD, who present with balance disturbances and increased risk for falls. The current thesis addresses gait characteristics in patients with COPD, to gain more insight into gait alterations in COPD, and the effect of pulmonary rehabilitation on gait characteristics in this patient population.

Three-dimensional motion analysis is considered as the gold standard for gait assessment. The Gait Real-time Analysis Interactive Lab (GRAIL) system combines 3-dimensional motion analysis, instrumented treadmill walking and virtual reality environment projected on a 180 degrees cylindrical screen. This novel system enables self-paced treadmill walking; the treadmill speed changes according to the subject's position on the treadmill. The 6-minute walk test (6MWT) is a self-paced test and is commonly used in clinical practice to measure functional exercise capacity, assess prognosis and evaluate response to treatment in patients with respiratory diseases. In addition, the 6MWT is used for health care decision making. The GRAIL system may therefore be an appropriate measure to assess the 6-minute walk distance. Chapter 2 demonstrated that patients with COPD walked further in the second GRAIL 6MWT. In addition, the GRAIL system is a valid and reproducible method to assess the walk distance during the 6MWT in patients with COPD and non-COPD subjects. The GRAIL system slightly underestimates the walk distance as compared to the overground 6MWT in patients with COPD. However, this system seems to provide walk distances closest to the overground 6MWT as compared to previous studies on treadmill-based 6MWTs. Consequently, the GRAIL system is a promising system to assess the 6-minute walk distance in patients with COPD and non-COPD subjects.

Different parameters are used to describe gait characteristics. The inherent fluctuations in gait characteristics can be evaluated in two ways: 1) via the variability of stride-to-stride fluctuations (objectified as standard deviation or coefficient of variation [CoV]), in which previous fluctuations are considered as independent from previous fluctuations, 2) via the patterns within stride-to-stride fluctuations that can identify the underlying control of gait characteristics. Both methods have been used to differentiate groups, such as fallers and non-fallers, or healthy from unhealthy subjects. In addition, stride-to-stride fluctuations can be calculated over spatiotemporal gait parameters (distance-time parameters), the motion of the center of mass, and kinematics (joint motion). Chapter 3 addresses the spatiotemporal gait characteristics in patients with COPD and non-COPD subjects during 
the GRAIL-based 6MWT. Patients with COPD demonstrate reduced walking speeds and stride lengths, and increased step times as compared to non-COPD subjects. The CoV of stride-to-stride fluctuations was altered in patients with COPD, including stride length, as compared to non-COPD subjects. A sub analysis of subjects with similar 6MWDs demonstrated that the $\mathrm{CoV}$ in stride length remained increased in patients with COPD. These findings suggest that patients with COPD performed the GRAIL-based 6MWT differently compared to non-COPD subjects.

Patients with COPD present with airway obstruction and often lower-limb muscle dysfunction. These factors may have a significant impact on functional exercise capacity in patients with COPD. Consequently, reduced lower-limb muscle strength may have a negative effect on gait characteristics in COPD. Consequently, differences in gait characteristics between patients with COPD with low quadriceps muscle strength and those with higher quadriceps muscle strength were assessed. Chapter 3 did not provide evidence for this reasoning. In addition, a reduced lung function has been considered a major problem in performing activities of daily living. Patients experience dyspnea during functional activities and this sensation increases with a prolonged duration or intensity of this activity. This chapter addresses the association between gait parameters and lung function as expressed by the forced expiratory volume in one second, used for stratifying the degree of airflow limitation in patients with COPD. Swing time seemed to be associated with a worse lung function.

Chapter 4 describes the stride-to-stride fluctuations in spatiotemporal gait characteristics and the center of mass movement in patients with COPD during the GRAIL-based 6MWT. Patients with COPD demonstrated increased mean stride length, increased variability in stride length, and increased regularity of patterns within stride length fluctuations as compared to non-COPD subjects when accounting for walking speed differences between the groups. These findings implicate that patients with COPD exhibit gait alterations as compared to non-COPD subjects. These gait alterations may be associated with increased fall risk and reduced balance control in patients with COPD. Though local dynamic stability of the center of mass did not differ between COPD and non-COPD subjects when accounting for walking speed differences, further studies are recommended to identify the relation between functional clinical parameters and gait characteristics.

In addition to spatiotemporal gait parameters, joint kinematics (joint angles) can be quantified via 3-dimensional motion analysis. Joint kinematics are more global parameters than spatiotemporal gait parameters, and could be altered in patients with COPD as compared to non-COPD subjects. Moreover, walking speed different from the preferred selfselected walking speed of the subject, may elicit gait alterations even further. Chapter 5 describes joint kinematics in the lower extremities between patients and non-COPD 
subjects, while walking at different walking speeds; at self-selected walking speed, $+20 \%$ and $-20 \%$ of the self-selected walking speed. Patients with COPD demonstrated reduced local dynamic stability in the knee and hip joint patterns across the walking speeds. Reduced local dynamic stability has been related to increased fall risk in older adults. This measure has also been suggested as a potential early indicator for fall risk. Therefore, reduced local dynamic stability in joint movement patterns in COPD, might be associate with increased balance issues and fall risk present in this patient population.

The effect of pulmonary rehabilitation on gait characteristics of patients with COPD is discussed in Chapter 6. A routine pre-rehabilitation assessment was conducted to determine amongst other things the physical condition, extra-pulmonary features and comorbidities, before attending a comprehensive pulmonary rehabilitation program consisting of 40 sessions supervised by an interdisciplinary team. Finally, a two-day outcome assessment was conducted to evaluate the effects of the rehabilitation program for each patient. Exercise training is a highly important component of pulmonary rehabilitation to strengthen muscle groups in the upper and lower extremities. Therefore, various training methods are embedded in the pulmonary rehabilitation program, including endurance, strength and interval training. This chapter showed that patients with COPD significantly improve the walked distance after pulmonary rehabilitation, with faster walking speeds and stride times. While quadriceps muscle strength and endurance increased and functional exercise capacity improved following pulmonary rehabilitation, no improvements in stride-to-stride fluctuations of the locomotor system were found in patients with COPD. In addition, differences in mean gait characteristics were found between good and poor responders, while stride-to-stride fluctuations were not discriminative. These findings indicate that the current PR does not alter stride-to-stride fluctuations in patients with COPD. Additional training programs specifically targeting balance and gait function may be beneficial in improving gait characteristics in patients with COPD.

In Chapter 7, the main findings of this thesis are discussed in light of current literature. This thesis shows that patients with COPD demonstrate altered gait characteristics during the self-paced treadmill 6MWT and while walking at different speeds. Furthermore, pulmonary rehabilitation improves lower-limb muscle function and functional exercise capacity in COPD. However, changes in gait characteristics, in particular those that are related to fall risk, were not found after pulmonary rehabilitation. The current thesis therefore provides a basis for further research in gait impairments in COPD in relation to balance and fall risk. These findings complement the understanding of gait impairments in COPD, which is important as COPD is a multisystemic disease affecting functional activities such as walking. In addition, this chapter describes the limitations of the current thesis and recommendations for future studies. We recommend to perform additional 
studies on identifying mechanisms contributing to reduced gait function in COPD, identifying diagnostic and monitoring tools to assess gait characteristics in COPD, and evaluating the relationship of gait alterations with respect to balance disturbances and falls in COPD. 

Samenvatting 
Chronische obstructieve longziekte (COPD) wordt gekenmerkt door aanhoudende luchtwegobstructie, met symptomen zoals kortademigheid en vermoeidheid. Daarnaast wordt COPD geassocieerd met extra-pulmonale manifestaties, waaronder skeletspier dysfunctie. Zowel de functionele inspanningscapaciteit als mobiliteit neemt af naarmate de ernst van COPD toeneemt. Patiënten met COPD ervaren beperkingen in de uitvoering van activiteiten in het dagelijks leven. Wandelen is een problematische activiteit in het dagelijks leven van patiënten met COPD. Dit is mogelijk geassocieerd met verstoringen in het gangpatroon, naast het feit dat patiënten met COPD meer balansverstoringen en een groter valrisico hebben in vergelijking met gezonde personen. Dit proefschrift richt zich op het in kaart brengen van het gangpatroon van patiënten met COPD, om zodoende meer inzicht te krijgen in de problematiek van het gangpatroon van patiënten met COPD, en het effect van longrevalidatie op het gangpatroon binnen deze populatie.

Driedimensionale bewegingsanalyse wordt als de gouden standaard gezien voor gangbeeldanalyse. De Gait Real-time Analysis Interactive Lab (GRAIL) combineert driedimensionale bewegingsanalyse met geïnstrumenteerde loopbanden binnen een virtuele omgeving. Dit systeem ontleent zich ook voor het zelf aansturen van de loopbandsnelheid. De 6-minuten wandeltest (6MWT) wordt in de klinische praktijk gebruikt om de inspanningscapaciteit van patiënten met COPD te evalueren. Het GRAIL-systeem kan mogelijk gebruikt worden voor het uitvoeren van de 6MWT. Hoofdstuk 2 toont aan dat het GRAILsysteem een valide en reproduceerbaar instrument is om de 6MWT uit te voeren in patiënten met COPD en niet-COPD personen. Ondanks dat het GRAIL systeem de wandelafstand ten opzichte van de reguliere grond 6MWT onderschat, benadert dit systeem de reguliere grond wandelafstand in tegenstelling tot voorgaande studies waar de 6MWT op een loopband werd uitgevoerd. Bovendien verleent het GRAIL-systeem om het gangbeeld in kaart te brengen om beter inzicht te verkrijgen in de verstoringen in het gangpatroon van patiënten met COPD.

Het gangbeeld kan middels verschillende parameters worden beschreven. De inherente fluctuaties in het gangpatroon kunnen op twee manieren in kaart worden gebracht: 1) middels de mate van variabiliteit, waarin elke fluctuatie onafhankelijk is van voorgaande fluctuaties, 2) middels de patronen in de temporale fluctuaties, om de onderliggende controle van het gangbeeld in kaart te brengen. Beide methoden worden gebruikt om groepen van elkaar te onderscheiden, waaronder vallers van niet-vallers, en gezonde personen van personen met een aandoening. De fluctuaties kunnen berekend worden over de spatio-temporele parameters, het massamiddelpunt van het lichaam en de kinematica (beweging van de gewrichten). Hoofdstuk 3 beschrijft de spatio-temporele gangparameters van patiënten met COPD in vergelijking met het gezonde niet-COPD personen tijdens de GRAIL 6MWT. Patiënten met COPD leggen een kortere wandelafstand af en ver- 
tonen grotere staplengtes, en langere staptijden in vergelijking met niet-COPD personen. Patiënten met COPD vertonen een grotere mate van variabiliteit in de schredelengte in vergelijking met niet-COPD personen. Een subanalyse van personen met een vergelijkbare 6-minuten wandelafstand toont aan dat de variabiliteit in schredelengte verhoogd blijft in patiënten met COPD. Deze bevindingen suggereren dat patiënten met COPD de GRAIL 6MWT anders uitvoeren dan personen zonder COPD.

Patiënten met COPD vertonen luchtwegobstructie en spierdysfunctie. Deze factoren worden geassocieerd met de inspanningscapaciteit in patiënten met COPD. Skeletspier dysfunctie heeft mogelijk een negatief effect op het gangbeeld in COPD. Dit heeft geleid tot de analyse van het gangbeeld tussen patiënten met lage quadriceps spierkracht en patiënten met een hoge quadriceps spierkracht. Hoofdstuk 3 bevestigt deze rationale echter niet. Daarnaast verhindert een verslechterde longfunctie het uitvoeren van activiteiten in het dagelijks leven. Patiënten ervaren kortademigheid tijdens het uitvoeren van activiteiten en deze kortademigheid kan toenemen naarmate de activiteit langer duurt of de intensiteit van de activiteit toeneemt. In Hoofdstuk 3 wordt de mogelijke associatie tussen luchtwegobstructie en gangbeeld tijdens de GRAIL 6MWT onderzocht. Uit de resultaten blijken dat de tijd in de zwaaifase wordt geassocieerd met een verslechterde longfunctie in COPD.

In Hoofdstuk 4 worden de patronen in de fluctuaties van spatio-temporele parameters en het massa middelpunt van het lichaam in patiënten met COPD tijdens de GRAIL 6MWT onderzocht. Patiënten met COPD vertonen veranderingen in schredelengte in vergelijking met niet-COPD personen. De gemiddelde schredelengte, de mate van fluctuaties in schredelengte, en patronen in fluctuaties van schredelengte zijn meer rigide in patiënten met COPD wanneer gecorrigeerd wordt voor het verschil in wandelsnelheid tussen de groepen. Dit duidt op verstoringen in het gangpatroon van patiënten met COPD. Deze veranderingen in het gangbeeld zijn mogelijk geassocieerd met een verhoogde valrisico en verminderde balanscontrole in patiënten met COPD. Ondanks dat de lokale stabiliteit van het massamiddelpunt van het lichaam niet aangedaan is in patiënten met COPD wanneer gecorrigeerd wordt voor het verschil in wandelsnelheid, wordt aangeraden om vervolgonderzoek te richten op het identificeren van de relatie tussen functionele klinische parameters en gangbeeld parameters.

Gewrichtshoeken van de onderste extremiteiten zijn meer globale parameters dan spatiotemporele parameters en zouden mogelijk aangedaan zijn in patiënten met COPD. Wanneer personen buiten de eigen voorkeurssnelheid wandelen, kan dit leiden tot grotere veranderingen in het gangpatroon. Hoofdstuk 5 beschrijft de gewrichtshoeken in de onderste extremiteiten van patiënten en niet-COPD personen, terwijl de deelnemers op verschillende snelheden hebben gewandeld. Patiënten met COPD worden gekenmerkt 
door een verminderde stabiliteit in de bewegingspatronen van de knie en heup over de verschillende wandelsnelheden in vergelijking met niet-COPD personen. In de algemene populatie, wordt een verminderde stabiliteit geassocieerd met een verhoogd valrisico. Dit duidt mogelijk op een relatie tussen een verminderde stabiliteit in de bewegingspatronen van het lichaam en de verhoogde balansverstoringen en valrisico in de COPD patiëntenpopulatie.

In Hoofdstuk 6 wordt het effect van longrevalidatie op het gangpatroon van patiënten met COPD beschreven. Patiënten met COPD hebben een longrevalidatie programma ondergaan van 40 sessies. Fysieke training, waaronder duur, kracht en interval training, is een belangrijk component binnen de reguliere longrevalidatie. Longrevalidatie heeft geresulteerd in een verbeterde wandelafstand in patiënten met COPD. Patiënten vertonen een snellere wandelsnelheid en schredetijd. Bovendien zijn de spierkracht en uithoudingsvermogen in de quadriceps spieren toegenomen. Echter vertonen patiënten met COPD geen veranderingen in de fluctuaties van de gangparameters na longrevalidatie. Dit betekent dat de huidige longrevalidatie programma tot dusver niet tot verbeteringen in de variabiliteit van het bewegingsapparaat in patiënten met COPD leidt. Daarnaast werden verschillen in gemiddelde spatiotemporale parameters aangetoond tussen patiënten die verbeteren tijdens de revalidatie en patiënten die niet-tot nauwelijks verbeteren. Echter werden er geen verschillen gevonden in de variabiliteit in het gangpatroon tussen deze subgroepen. Additionele trainingsprogramma's gericht op balans en loopfunctie zouden mogelijk tot verbeteringen kunnen leiden in het gangbeeld in patiënten met COPD.

In Hoofdstuk 7 worden de bevindingen van het proefschrift besproken en tegen het licht gehouden met de huidige literatuur. Dit proefschrift laat zien dat patiënten met COPD veranderingen in het gangpatroon vertonen tijdens de 6MWT op het GRAIL systeem en het wandelen op verschillende wandelsnelheden. Daarnaast heeft longrevalidatie een positieve invloed op spierfunctie van het onderlichaam en de inspanningscapaciteit in patiënten met COPD. Echter, heeft longrevalidatie geen invloed op de gangparameters, die geassocieerd worden met balansverstoringen en een verhoogd valrisico. In dit hoofdstuk worden de zwakheden van het proefschrift besproken en worden aanbevelingen voor toekomstig onderzoek gedaan. Toekomstig onderzoek kan zich richten op het identificeren van de onderliggende mechanismen die een rol spelen in een verslechterd gangbeeld in COPD, het identificeren van diagnostische en monitorings-instrumenten om het gangbeeld van patiënten met COPD in kaart te brengen, en het evalueren van de relatie tussen veranderingen in het gangbeeld met balansverstoringen en valrisico in COPD. 


\section{Valorisation}


This thesis includes a series of scientific studies to broaden our knowledge on gait characteristics of patients with chronic obstructive pulmonary disease (COPD). In the current chapter, these studies and their outcomes are positioned in a broader societal and economical context to transfer the scientific knowledge described into clinical practice, and to translate the findings into future perspectives.

\section{Relevance}

COPD is highly prevalent and is expected to further increase in the coming decades. COPD is a leading cause of morbidity and mortality worldwide. Moreover, COPD has a major societal and economic burden, due to factors such as frequent exacerbations and the presence of comorbidities in patients with COPD. COPD has a significantly impact on a patient's quality of life and those caring for the patient. In addition, the decline in respiratory function characterizes COPD and is associated with progressive symptoms and functional impairment.

Patients with COPD suffer from skeletal muscle dysfunction and functional limitations, which have a significant effect on the patients' daily life. Skeletal muscle dysfunction and functional limitations worsen with increasing disease severity. Functional limitations result in reduced independence and quality of life in patients with COPD. Consequently, activities of daily life, such as walking, become increasingly more difficult to perform. Furthermore, patients with COPD are at increased risk for falls as compared to non-COPD individuals. As falls often occur during walking, it is important to gain a better understanding of gait impairments in COPD. Consequently, improved insight into gait impairments in COPD can contribute to developing assessment tools to evaluate gait function and providing interventions to reduce gait impairments in patients with COPD.

\section{Target groups}

\section{Health care professionals}

The results of this thesis are primarily important for health care providers, such as respiratory physicians and physiotherapists. Increasingly more studies report gait alterations in patients with COPD as compared to non-COPD individuals and the current findings presented in this thesis strengthen previous findings on gait alterations in COPD. While gait assessment is currently not incorporated in clinical practice for COPD, it may be a promising tool to improve patient's care. Therefore, this thesis contributes to the in- 
creasing awareness of the extra-pulmonary manifestations of COPD, including reduced mobility and gait function. It discusses the opportunities of gait analysis and its clinical implications in the respiratory field. Identifying gait impairments in patients with COPD is important to determine appropriate diagnostic tools to assess gait function in this population. In addition, gait characteristics that are associated with fall risk are of clinical interest and gait analysis in COPD may help deciding treatment methods for those with impaired gait function. Consequently, gait assessment and gait training may become an essential part of disease management in COPD.

\section{Patients with COPD}

Patients do not directly benefit from the studies described in this thesis. However, this thesis sheds light on gait assessment and evaluation of gait function after pulmonary rehabilitation in COPD. On the long term, patients may profit from a better understanding of gait impairments in COPD. Diagnostic tools for gait impairments may be developed and training programs directed to improve gait function in COPD may be introduced in the treatment of patients. Such gait training exercises could be integrated in pulmonary rehabilitation programs to provide personalized care to each patient.

This thesis showed that patients with COPD benefit from a comprehensive pulmonary rehabilitation program. More specifically, lower-limb muscle strength and endurance, body composition, mobility, functional exercise capacity and walking speed improve following pulmonary rehabilitation. These findings are in line with the current literature on the benefits of pulmonary rehabilitation for patients with COPD.

\section{Activities and products}

The findings of this thesis have led to several activities in the field of expertise. The results of this thesis have been presented at various symposia and congresses, including the European Respiratory Society Congress in 2014 (Munich, Germany), 2015 (Amsterdam, the Netherlands), and 2016 (London, United Kingdom); the SMALLL congress in 2015 (Maastricht, the Netherlands) and 2017 (Leuven, Belgium); the World Congress of Biomechanics in 2018 (Dublin, Ireland); and the Netherlands Respiratory Society Symposium in 2015. Furthermore, the findings have been translated into original manuscripts, published in international scientific journals. Results have been presented at different meetings at CIRO, Maastricht University, Maastricht University Medical Centre and other institutions. These activities have led to a pilot study aimed at gait perturbation in patients with COPD. To gain expertise from a renowned center in gait variability, this has led 
to an international collaboration to study the underlying patterns within gait fluctuations in COPD, at the Center for Research in Human Movement Variability at the University of Nebraska in Omaha (US). This thesis may inspire future research in understanding gait characteristics in patients with COPD and determining methods to evaluate and improve gait function in COPD.

\section{Future perspectives}

The findings in the current thesis might be important for researchers in the field. This thesis described alterations in gait characteristics of patients with COPD. This has led to new research questions, which are presented in the discussion chapter. The presented findings create new opportunities and calls for future studies. Studies into gait impairments in COPD in various conditions are needed, and investigations in the underlying causes of gait impairments and its relation to balance and fall risk in COPD are recommended. Furthermore, studies into methods (e.g. exercise, gait or balance training) to improve gait characteristics in patients with COPD is of interest to improve gait function in these patients.

In addition, gait analysis was conducted using three dimensional motion capture systems, the gold standard for gait assessment. In the near future, studies may investigate the use of portable and less expensive systems to quantify and monitor gait characteristics in COPD. This creates the opportunity to evaluate and monitor gait in patients with COPD in their home environment. In turn, gait assessment may become more accessible in clinical practice, enabling implementation of gait assessment in standard care for COPD. 
Dankwoord 
$\mathrm{Nu}$ is het moment aangebroken om iedereen te bedanken die hebben bijgedragen aan dit proefschrift. Zonder jullie steun en medewerking heeft dit proefschrift niet tot stand kunnen komen.

Allereest wil ik alle patiënten bedanken voor jullie deelname aan mijn onderzoek. Ik heb veel respect voor het feit dat jullie je belangeloos hebben ingezet voor de wetenschap, naast de klachten die jullie dagelijks ervaren en alle onderdelen van het revalidatieprogramma. Een grote dank gaat ook uit naar alle gezonde vrijwilligers, die hebben deelgenomen aan het onderzoek.

De Raad van Bestuur van CIRO, prof. Wouters en Ingrid Augustin, wil ik beiden graag bedanken voor de ondersteuning van mijn promotieonderzoek. Daarbij wil ik mijn tweede promotor, prof. Wouters, in het bijzonder bedanken voor zijn inzicht en kritische commentaar op de artikelen die ik de afgelopen jaren heb geschreven.

Graag bedank ik mijn promotor, Martijn, voor de begeleiding en betrokkenheid tijdens mijn promotieonderzoek. Ik heb bewondering voor jouw enthousiasme en gedrevenheid in wetenschappelijk onderzoek. Onder jouw supervisie heb ik veel geleerd. Bedankt voor jouw geduld en vertrouwen.

Ik ben dankbaar voor mijn copromotor, Kenneth, voor zijn begeleiding, steun en vertrouwen in de afgelopen jaren. Ik kon met mijn wetenschappelijke en persoonlijke vragen altijd bij jou terecht. De PhD meetings die je hebt geïntroduceerd waren zeer waardevol. Jouw kennis en inzicht waren van groot belang voor dit proefschrift. Bedankt voor deze kans.

Dear Jenna, thank you for your guidance during my fellowship at the University of Nebraska at Omaha. The moment I have contacted you, I never expected that a few months later this would lead to an inspiring research fellowship at the department of Biomechanics. You made me feel welcome and I'm very grateful for your hospitality and expertise in biomechanics.

Graag bedank ik alle co-auteurs voor de prettige samenwerking, waaronder Sjoerd, jouw snelle en kritische feedback is zeer waardevol. Frits, bedankt voor jouw bijdrage en klinische blik op mijn artikelen. Dear Kendra, thank you for your expertise in statistics and your valuable feedback on the manuscript.

De beoordelingscommissie: prof. dr. Hans Savelberg, prof. dr. Lodewijk van Rhijn, prof. dr. Mirjam Pijnappels, prof. dr. Ton Lensen, en dr. Alex van 't Hul, wil ik hartelijk bedanken voor het lezen en beoordelen van mijn proefschrift. Ook de promotiecommissie wil ik bedanken voor het lezen van mijn proefschrift, jullie aanwezigheid tijdens mijn verdediging en de kritische vragen.

Alle CIRO-medewerkers, bedankt voor de prettige werksfeer en jullie betrokkenheid. Een 
speciale dank gaat uit naar de afdeling longfunctie, radiologie en biometrie. Jullie flexibiliteit en inzet voor de onderzoeksmetingen waardeer ik zeer. Daarnaast wil ik de collega's bedanken die zelf aan het onderzoek hebben deelgenomen en / of enthousiaste vrijwilligers hebben aangeleverd.

Mijn collega's van het onderzoek in CIRO, oftewel de Gezelligheidshok: Anouk, Carmen, Cindy, Coby, Dionne B, Dionne S, Esther, Fiona, Jeannet, Nienke, Rafael, Sarah, Yvonne en Vasilis. Jullie maakten promoveren toch zoveel leuker. Ik denk met veel plezier terug aan de leuke momenten die we samen hebben gedeeld (etentjes, bruiloften, kraambezoeken, congressen en de wall-sit challenge). In het bijzonder wil ik Jeannet bedanken voor haar hulp bij het uitvoeren van de onderzoeksmetingen. Anouk en Jeannet, ik ben blij dat onderzoek met de GRAIL wordt voortgezet in CIRO.

Nu zijn jullie aan de beurt, collega's van de uni, waaronder Bas, Bernard, Brenda, Chris, Hans, HQ, Irene, Jasper, Kyra, Li-Juan, Michiel, Pieter, Thamar en Wouter. Het begon in kamer 2.220, met wat veranderingen in de samenstelling, en eindigde in kamer 2.200. Ik heb een geweldige tijd met jullie gehad. Bedankt voor de leuke werksfeer. Jullie interesse, kritische vragen en behulpzaamheid hebben mij altijd erg geholpen tijdens mijn promotieonderzoek. Paul wil ik bedanken voor de technische ondersteuning, het programmeren en de leuke gesprekken over het goede leven. Ook Harry wil ik bedanken voor het feit dat je mij altijd uit te brand hielp als ik computerproblemen had.

De studenten die hebben bijgedragen aan mijn onderzoek. Bedankt voor jullie hulp met het includeren van proefpersonen, het verrichten van de onderzoeksmetingen en het meedenken. Ik wens jullie veel succes toe in jullie toekomst.

Mijn huidige collega's bij Orthopedie Groot Eindhoven, dank voor jullie geduld en interesse in de voortgang van dit proefschrift. Ik ben blij dat ik deze nieuwe uitdaging mocht aangaan tijdens het afronden van mijn promotieonderzoek. Met veel plezier ga ik dagelijks naar mijn werk. In het bijzonder wil ik Marieke bedanken voor haar steun en luisterend oor.

Mijn Fyrfad maatjes waaronder Leonie, Lisa, Sara, Sanne en Vera. Ik kijk met veel plezier terug naar alle mooie seizoenen en gezellige activiteiten. Onze yell blijft mij altijd dierbaar. Liza en Martine, dank voor de gezelligheid en afleiding tijdens mijn promotieonderzoek. Aan mijn oud-teamgenoten van Nuvoc D3 en huidige teamgenoten van Nuvoc D2, tijdens het volleyballen kon ik mijn gedachten verzetten. Bedankt voor de afleiding en leuke momenten binnen en buiten het veld.

Mijn studievriendinnen uit Maastricht: Daphne, Iris, Marleen, Ruth en Sanne. Dank voor jullie steun. Ik kijk elke keer uit naar het volgende moment dat wij samen komen om bij te kletsen. Fabienne, jouw nuchterheid en grappige opmerkingen waardeer ik ontzettend. 
Bedankt voor de interesse in de voortgang van mijn proefschrift en de gezellige etentjes om mijn gedachten af te leiden.

Corine, Hilde, Lieve, Lysanne, Sophie en Suus, jullie ken ik al vanaf de middelbare school en ondanks de afstand hebben we contact met elkaar gehouden. We hebben al veel mooie momenten mogen meemaken met elkaar. Bij jullie voel ik me altijd thuis. Bedankt voor jullie steun, geduld en vriendschap.

Nu een speciaal woord van dank aan mijn paranimf, Aniek. Sinds onze eerste studentenjaar in Maastricht zijn we vriendinnen. Samen kletsen, volleyballen, dansjes doen, eten, en onze blijdschap en frustraties over promoveren met elkaar delen. Je bent onmisbaar. Nu jouw proefschrift ook klaar is, kunnen we onze city-trip eindelijk plannen!

Toos, Mathieu, Maarten en Lidia, bedankt voor jullie interesse in de voortgang van dit proefschrift. Ik waardeer het ontzettend dat jullie altijd voor ons klaar staan.

親愛的爸爸、媽媽，

在一個陌生國家展開新生活並非易事。我在您們身上學懂努力不解，從而達到今日的成就。 感激您們一直支持，經常準備家常菜為我打氣。謝謝您們無條件的愛。

Lieve Hoi-Yan, Felix, Karleun, Tiffany, Che-Yan en Huy. Jullie gaven mij alle ruimte om mijn promotieonderzoek af te ronden. Ik ben dankbaar dat ik altijd bij jullie terecht kan. Ik heb het getroffen met zo'n leuke familie!

Lieve Martijn, tijdens mijn promotieonderzoek stond je altijd voor mij klaar. Op de momenten waarop ik het even niet meer zag zitten, kon je mij ook geruststellen en weer aan het lachen maken. Woorden schieten tekort hoeveel je voor mij betekent. Ik hoop voor jou ook zo'n goede steun te zijn tijdens het afronden van jouw promotieonderzoek. Ik kijk uit naar wat we samen nog meer gaan beleven! 
Curriculum vitae 
Wai-Yan Liu is op 17 november 1988 geboren te Bladel en Netersel. Zij volgde haar voortgezet onderwijs aan het Pius X-college in Bladel. Vervolgens heeft ze van 2007-2011 aan de Universiteit van Maastricht de bachelor Gezondheidswetenschappen gestudeerd. De master in Physical Activity and Health: Biology of Human Performance and Health aan de Universiteit van Maastricht heeft ze in 2011 afgerond. Hierna is zij als onderzoeksassistent op de afdeling Bewegingswetenschappen bij de knie artrose studie aan de slag gegaan.

In 2012 startte ze haar promotieonderzoek bij CIRO, een expertisecentrum voor chronisch orgaanfalen, en de afdeling Voeding en Bewegingswetenschappen aan de Universiteit van Maastricht. Ze heeft onderzoek gedaan naar het gangpatroon van patiënten met een chronisch obstructieve longziekte. Gedurende deze periode heeft zij haar onderzoeksresultaten op verschillende (inter)nationale congressen gepresenteerd. Daarnaast heeft zij een Short Term International Research Fellowship toegewezen gekregen van het European Respiratory Society om zich te verdiepen in verstoringen in het gangpatroon van personen met een chronisch obstructieve longziekte bij de afdeling Biomechanica, the Center of Human Movement Variability, aan de Universiteit van Nebraska in Omaha (VS) onder begeleiding van dr. Yentes.

Sinds juli 2018 werkt ze als onderzoekscoördinator bij Orthopedie Groot Eindhoven in het Máxima Medisch Centrum en het Catharina Ziekenhuis in Eindhoven. 
List of publications 
Liu WY, Meijer K, Delbressine JM, Willems PJ, Wouters EFM, Spruit MA. Effects of Pulmonary Rehabilitation on Gait Characteristics in Patients with COPD. J. Clin. Med. 2019;8:459.

Liu WY, Schmid KK, Meijer K, Spruit MA, Yentes JM. Patients with COPD walk with less consistent organization of movement patterns of the lower extremity. Respiratory Care. In press.

Liu WY, Spruit MA, Delbressine JM, Willems PJ, Yentes JM, Bruijn SM, Franssen FME, Wouters EFM, Meijer K. Alterations in stride-to-stride fluctuations in patients with chronic obstructive pulmonary disease during the self-paced treadmill 6-minute walk test (submitted for publication).

Meijer K, Oomen PW, Garcia van der Westen R, Liu WY, van Rhijn LW, Emans PJ, Drost MR. The mediolateral component of knee joint loading as predicted by musculoskeletal models is increased in patients with mild knee osteoarthritis (submitted for publication).

Oomen PW \& Verlaan L, Schmitz T, Liu WY, Peters MJM, Emans PJ, van Rhijn LW, Drost MR, Meijer K. Obese knee OA patients have increased knee adduction moments during gait (submitted for publication).

Verlaan, L, Vangeneugden J, Oomen PW, Liu WY, Peters MJM, Natour N, Emans PJ, Meijer K. Signatures of knee osteoarthritis in the temporal and fractal dynamics of human gait (submitted for publication).

Verlaan L, Boekesteijn RJ, Oomen PW, Liu WY, Peters MJM, Emans PJ, van Rhijn LW, Meijer K. Knee adduction moments are not increased in obese knee osteoarthritis patients during stair negotiation. Gait Posture. 2019;16;73-:154-160.

Verlaan L, Boekesteijn RJ, Oomen PW, Liu WY, Peters MJM, Witlox MA, Emans PJ, van Rhijn LW, Meijer K. Biomechanical Alterations during Sit-to-Stand Transfer Are Caused by a Synergy between Knee Osteoarthritis and Obesity. Biomed Res Int. 2018;2018:3519498.

Liu WY, Spruit MA, Delbressine JM, Willems PJ, Franssen FM, Wouters EFM, Meijer K. Spatiotemporal gait characteristics in patients with COPD during the Gait Real-time Analysis Interactive Lab-based 6-minute walk test. PLoS One. 2017;12(12):e0190099.

Liu WY, Meijer K, Delbressine JM, Willems PJ, Franssen FM, Wouters EFM, Spruit MA. Reproducibility and validity of the 6-minute walk test using the Gait Real-time Analysis 
Interactive Lab in patients with COPD and controls. PLoS One. 2016;11(9):e0162444.

McCrum C, Essers J, Jie LJ, Liu WY, Meijer K. Commentary: Older adult can improve compensatory stepping with repeated postural perturbations. Front Aging Neurosci. 2016;8(111). 